\title{
Advancing Tin Halide Perovskites: Strategies Toward ASnX3 Paradigm for Efficient and Durable Optoelectronics
}

Yan, Yajie; Pullerits, Tonu; Zheng, Kaibo; Liang, Ziqi

Published in:

ACS Energy Letters

Link to article, DOI:

10.1021/acsenergylett.0c00577

Publication date:

2020

Document Version

Peer reviewed version

Link back to DTU Orbit

Citation (APA):

Yan, Y., Pullerits, T., Zheng, K., \& Liang, Z. (2020). Advancing Tin Halide Perovskites: Strategies Toward ASnX3 Paradigm for Efficient and Durable Optoelectronics. ACS Energy Letters, 5(6), 2052-2086.

https://doi.org/10.1021/acsenergylett.0c00577

\section{General rights}

Copyright and moral rights for the publications made accessible in the public portal are retained by the authors and/or other copyright owners and it is a condition of accessing publications that users recognise and abide by the legal requirements associated with these rights.

- Users may download and print one copy of any publication from the public portal for the purpose of private study or research.

- You may not further distribute the material or use it for any profit-making activity or commercial gain

- You may freely distribute the URL identifying the publication in the public portal 


\title{
Review
}

\section{Advancing Tin Halide Perovskites: Strategies Toward ASnX3 Paradigm for Efficient and Durable Optoelectronics}

\author{
Yajie Yan, Tõnu Pullerits, Kaibo Zheng, and Ziqi Liang
}

ACS Energy Lett., Just Accepted Manuscript • DOI: 10.1021/acsenergylett.0c00577 • Publication Date (Web): 18 May 2020

Downloaded from pubs.acs.org on May 25, 2020

\section{Just Accepted}

"Just Accepted" manuscripts have been peer-reviewed and accepted for publication. They are posted online prior to technical editing, formatting for publication and author proofing. The American Chemical Society provides "Just Accepted" as a service to the research community to expedite the dissemination of scientific material as soon as possible after acceptance. "Just Accepted" manuscripts appear in full in PDF format accompanied by an HTML abstract. "Just Accepted" manuscripts have been fully peer reviewed, but should not be considered the official version of record. They are citable by the Digital Object Identifier (DOI®). "Just Accepted" is an optional service offered to authors. Therefore, the "Just Accepted" Web site may not include all articles that will be published in the journal. After a manuscript is technically edited and formatted, it will be removed from the "Just Accepted" Web site and published as an ASAP article. Note that technical editing may introduce minor changes to the manuscript text and/or graphics which could affect content, and all legal disclaimers and ethical guidelines that apply to the journal pertain. ACS cannot be held responsible for errors or consequences arising from the use of information contained in these "Just Accepted" manuscripts. 


\title{
Advancing Tin Halide Perovskites: Strategies Toward $\mathrm{ASnX}_{3}$ Paradigm for Efficient and Durable Optoelectronics
}

\author{
Yajie Yan ${ }^{\dagger}$, Tõnu Pullerits ${ }^{\perp}$, Kaibo Zheng ${ }^{\ddagger, \perp^{*}}$, and Ziqi Liang ${ }^{\dagger *}$ \\ †Department of Materials Science, Fudan University, Shanghai 200433, China \\ Email: zqliang@fudan.edu.cn
}

Department of Chemistry, Technical University of Denmark, DK-2800 Kongens Lyngby, Denmark

Email: kzheng@,kemi.dtu.dk

${ }^{\perp}$ Department of Chemical Physics and NanoLund, Lund University, 22100 Lund, Sweden

\begin{abstract}
Various less-toxic substitutes for the $\mathrm{Pb}^{2+}$-based perovskites have been proposed wherein $\mathrm{Sn}^{2+}$-based metal halide perovskites (namely, Sn-PVSKs) hold the best prospect due to their comparable optoelectronic properties to $\mathrm{Pb}$ analogues. Nevertheless, the intrinsic instability and unfavorable crystallization of Sn-PVSKs place restrictions on both the device performance/durability and the fabrication reproducibility/large-scale manufacturing, respectively. Therefore, numerous attempts have been directed at exploring the underlying mechanisms of SnPVSKs and acquiring high-quality, ambient-stable thin films. In this review, a retrospect is firstly given on the milestones and general properties of paradigm $\mathrm{ABX}_{3}$ structured Sn-PVSKs. Then, their electronic structure evolution, photo-physics process and degradation pathways are thoroughly interpreted. The gained understanding triggers various strategies exploited in the categories of synthetic conditions, compositions, phase components as well as device architecture for diverse optoelectronic applications. The final section summarizes key advances in Sn-PVSKs and meanwhile offers the guidance for future improvements that depends critically on these methodologies.
\end{abstract}




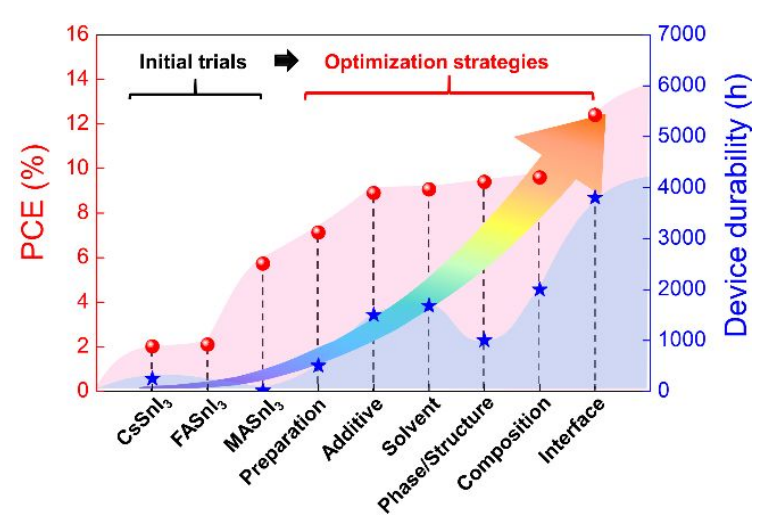

Ever since the initial establishment in $2009,{ }^{1}$ perovskites solar cells (PSCs) have made unprecedented progress. The device performance is skyrocketing with the power conversion efficiency (PCE) exceeding 25\% and the ambient device durability already over 10,000 hours. ${ }^{2}$ This demonstrates its maturity of technology for the next generation optoelectronics. ${ }^{3-5}$ The nominal chemical formula of metal halide perovskites (PVSKs) is $\mathrm{ABX}_{3}$. Based on the Goldschmidt tolerance factor ${ }^{6}$ and octahedral factor, ${ }^{7}$ the selection of A-sites fall within the range of organic/inorganic cations such as typically used $\mathrm{CH}_{3} \mathrm{NH}_{3}^{+}\left(=\mathrm{MA}^{+}\right), \mathrm{CH}\left(\mathrm{NH}_{2}\right)_{2}^{+}\left(=\mathrm{FA}^{+}\right), \mathrm{Cs}^{+}$ and so on, the B-site candidates include group 14 metal cations such as $\mathrm{Pb}^{2+}, \mathrm{Sn}^{2+}$ and $\mathrm{Ge}^{2+}$, and the $\mathrm{X}$ represent halide or pseudo-halide monovalent anions such as $\mathrm{Cl}^{-}, \mathrm{Br}^{-}, \mathrm{I}^{-}$and $\mathrm{SCN}^{-}$. By rationally tailoring the composition and stoichiometry of PVSKs, the optimal PCE of lead (Pb)based PSCs (Pb-PSCs) has reached 25.2\%. ${ }^{8}$

The crucial challenge towards commercialized applications is the toxicity of $\mathrm{Pb}$, which necessitates its viable replacement while maintaining decent performance and stability of devices. Attentions have been directed at exploring either novel lead-free substructures or $\mathrm{Pb}$ substitutions in $\mathrm{ABX}_{3}$ type PVSKs. In the former efforts, three main categories of derivatives generated from aliovalent replacement of $\mathrm{Pb}^{2+}$ have been proposed with enhanced air and moisture tolerance as shown in Figure 1..$^{9-14}$ Nevertheless, these structures exhibited inferior photovoltaic performance to their parent $\mathrm{ABX}_{3}$ structure, which is attributed to the difficulties in synthesis or undesirable 
electronic structures including wide and indirect optical bandgaps $\left(E_{\mathrm{g}} \mathrm{s}\right)$.

On the other hand, the isovalent congeners of $\mathrm{Pb}^{2+}$ with less toxicity have been investigated both experimentally and theoretically, among which $\mathrm{Sn}^{2+}$ based PVSKs (denoted as Sn-PVSKs) manifest the best alternative with high charge-carrier mobilities and narrow $E_{\mathrm{g}} \cdot{ }^{15}$ In 2014 , as an experimental proof, the $\mathrm{MASnI}_{3-\mathrm{x}} \mathrm{Br}_{\mathrm{x}}$ and $\mathrm{MASnI}_{3}$ based PSCs demonstrated promising PCEs of $5.73 \%{ }^{16}$ and $6.4 \%,{ }^{17}$ respectively. However, the oxygen sensitive nature and uncontrollable film formation emerge as hurdles for $\mathrm{Sn}^{2+}$ based optoelectronics to fulfill the potential. From the perspective of valence electron configuration, the $6 \mathrm{~s}^{2}$ electrons of $\mathrm{Pb}$ atoms are difficult to lose as a result of inert electron pair effect induced by Lanthanide shrinkage, which does not exist in the fifth period and thus the $5 \mathrm{~s}^{2}$ electrons of $\mathrm{Sn}$ is unstable confronting strong electronegative matters such as oxygen. Therefore, $\mathrm{Sn}^{2+}$ is thermodynamically less stable than $\mathrm{Sn}^{4+}$, which results in the $\mathrm{Sn}^{2+}$ vacancies in Sn-PVSKs upon oxidation and induces the undesirable self-doping effect. In order to maintain this metastable state under ambient atmosphere for reproducible film preparation and normal device operation, increasing attempts have been concentrated on optimizing the synthetic conditions (i.e., additive, solvent or preparation methods), elemental compositions, device architecture as well as phase composition to kinetically stabilize Sn-PVSKs, which have successfully boosted the PCE up to $13.24 \%{ }^{18}$ and over $20 \%,{ }^{19,20}$ respectively, for neat Sn and binary $\mathrm{Sn} / \mathrm{Pb}$ systems, with the former demonstrating an outstanding stability of $3,800 \mathrm{~h}$ in inert atmosphere. $^{21}$

Motivated by recent intensive efforts and great success in lead-free PVSKs, to the best of our knowledge, approximately 13 review articles haven been published on the topic. These reviews have focused on the designing rules, ${ }^{22,23}$ preparation methods, ${ }^{24-27}$ structural diversities, ${ }^{28-32}$ and the resulting optoelectronic properties, ${ }^{33,34}$ mostly based on lead-free derivatives of $\mathrm{ABX}_{3}$ typed 
PVSKs. However, few reviews unraveled the intimate correlations among thin-film forming kinetics, interface engineering and device performance of the mostly studied Sn-PVSKs, which is indeed critical to further improving both stability and efficiency. To fill this gap, this article seeks to offer a comprehensive yet critical review of Sn-based optoelectronics. Firstly, we will retrospect on the milestones and general properties of Sn-PVSKs based on dimensionalities and A-site cations. Next, the deterministic mechanisms in Sn-based optoelectronic devices such as electronic structure evolution, photophysics process, and degradation pathways will be discussed. Based on an understanding of the above underlying processes in Sn-PVSKs, various strategies will be presented to prepare high-quality and oxidation-resistant Sn-PVSKs thin films for diverse optoelectronic applications in PSCs, light-emitting diodes (LEDs) and photodetectors (PDs) with an emphasis on their relations to device performance and stability, which leads us to the conclusion and outlooks for the guidance of future optimization that depends critically on the strategies employed.

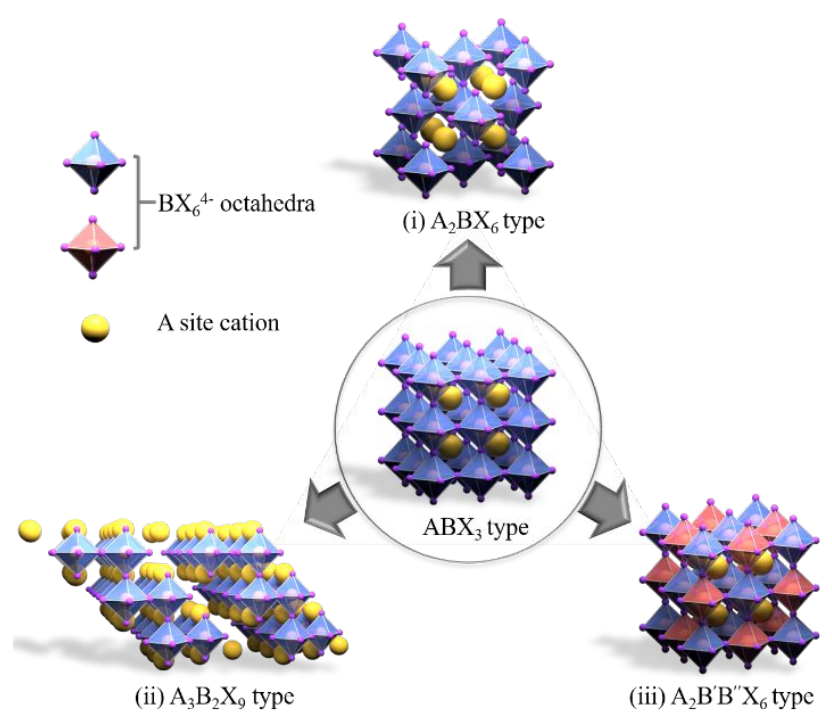

Figure 1. Schematic illustrations of $\mathrm{ABX}_{3}$ typed PVSKs and derivatives generated from heterovalent substitution of $B$ site divalent cation. The substructures feature a general composition of $i$ ) $\mathrm{A}_{2} \mathrm{BX}_{6}$ (i.e., vacancy-ordered double perovskites, $\mathrm{B}=\mathrm{Sn}^{4+}, \mathrm{Te}^{4+}$, etc.), ii) $\mathrm{A}_{3} \mathrm{~B}_{2} \mathrm{X}_{9}$ (i.e., twodimensional layered perovskites, $\mathrm{B}=\mathrm{Bi}^{3+}, \mathrm{Sb}^{3+}$ ), or iii) $\mathrm{A}_{2} \mathrm{~B}^{\prime} \mathrm{B}^{\prime \prime} \mathrm{X}_{6}$ (i.e., elpasolites, $\mathrm{B}^{\prime}=\mathrm{Bi}^{3+}, \mathrm{Sb}^{3+}$; $\mathrm{B}^{\prime \prime}=\mathrm{Au}^{+}, \mathrm{Ag}^{+}, \mathrm{Cu}^{+}, \mathrm{K}^{+}$, etc.). 
3D Sn-PVSKs. Given the comparable ion radius between $\mathrm{Pb}^{2+}(119 \mathrm{pm})$ and $\mathrm{Sn}^{2+}(112 \mathrm{pm})$, the $\mathrm{ASnX}_{3}$ adopted similar crystal structure as $\mathrm{Pb}$ analogue. Typically, the inorganic framework of metal-halide $\mathrm{ABX}_{3}$ perovskites consisted of divalent cations with six-fold coordination of halide monovalent anions. The corner-sharing $\left[\mathrm{BX}_{6}\right]^{4-}$ octahedra constructed inorganic $3 \mathrm{D}$ networks with twelve-coordinated A-site cations centering in the cages formed by eight neighboring octahedra. Empirically, the crystallographic symmetry and the lattice stability of this structure are evaluated by Goldschmidt tolerance factor ${ }^{6}$ as expressed by $t=\left(\mathrm{r}_{\mathrm{A}}+\mathrm{r}_{\mathrm{X}}\right) /\left[2^{1 / 2}\left(\mathrm{r}_{\mathrm{B}}+\mathrm{r}_{\mathrm{X}}\right)\right]$, in which $\mathrm{r}_{\mathrm{A}}, \mathrm{r}_{\mathrm{B}}$ and $\mathrm{r}_{\mathrm{X}}$ represent the radius of $\mathrm{A}, \mathrm{B}$ and $\mathrm{X}$ site ions, respectively. The $\mathrm{ABX}_{3}$ structure, if exists, possesses a reduction of symmetry from cubic to orthorhombic lattice when $t$ declines from unity and $0.813 .^{7}$ When the $\mathrm{A} / \mathrm{B}$ site cation becomes smaller/larger, the $\mathrm{ABX}_{3}$ structure is more distorted as $t$ decreases and finally collapse into non-perovskites. In the case of Sn-PVSKs, the A-site cation should range from $\sim 140$ to $\sim 220 \mathrm{pm}$ to preserve phase stability, ${ }^{35}$ which is compatible with $\mathrm{MA}^{+}$ $(217 \mathrm{pm})$ and $\mathrm{Cs}^{+}(167 \mathrm{pm}){ }^{36}$ The larger $\mathrm{FA}^{+}(253 \mathrm{pm})^{36}$ can also applicable except for slight distortions within the inorganic framework. Note that the tolerance factor itself is not a sufficient condition to predict the formation of perovskites. Another factor, namely, the octahedral factor, is also necessary for probing the formation of $\mathrm{ABX}_{3}$ structure, which is determined to range between 0.414 and 0.895 for stabilization of the $\left[\mathrm{BX}_{6}{ }^{-}\right]$units. ${ }^{37}$ Based on the above criteria, the A-site cations play an important role in phase stability of perovskites, exhibiting different optical absorption from their $\mathrm{Pb}$ counterparts (Figure 2A). Moreover, it can be seen from Figure 2B and C that the $E_{\mathrm{g}} \mathrm{s}$ of both $\mathrm{Sn}$ and Pb-PVSKs fall between 1.2-1.6 eV, whereas the absorption onset of Sn-PVSKs shows a strong dependence on the preparation method, which in part explains poor reproducibility of Sn-based devices. In this section, we will give a brief overview on basic 
properties and summarize the development history based on the selection of A-site cation.

MA-Sn-X PVSKs: In the first attempts, both Kanatzidis ${ }^{16}$ and Snaith ${ }^{17}$ teams demonstrated the fabrication of solution-processed $\mathrm{MASnI}_{3}$ based photovoltaic devices with impressive PCEs around $6 \%$ in a typical $\mathrm{n}-\mathrm{i}-\mathrm{p}$ architecture. Then, the comparable carrier diffusion length to $\mathrm{Pb}-$ PVSKs and the slow hot carrier cooling process in $\mathrm{MASnI}_{3}$ were confirmed, implying the promising potential in lead-free PSCs. ${ }^{38}$ However, the Sn-based optoelectronic devices were found extremely sensitive to oxygen, leading to inferior performance to that of $\mathrm{Pb}$ analogues. Therefore, extensive attentions have been paid upon the stability issues of MA-Sn-X PVSKs. By using density functional theory (DFT) calculation, Hu and coworkers revealed that the degradation of $\mathrm{MASnI}_{3}$ is attributed to the reaction of $\mathrm{Sn}-\mathrm{I}$ bond with ambient gas molecules to form new $\mathrm{H}-\mathrm{I}$ and $\mathrm{Sn}-\mathrm{O}$ bonds. Moreover, they found that the (011) surface was the most stable facet in $\mathrm{MASnI}_{3}$ against moisture and oxygen. ${ }^{39}$ Based on this work, Jiang and coworkers developed a novel two-step cation exchange method to enhance the (011) orientation, which remarkably suppressed the oxidation of $\mathrm{Sn}^{2+}$ and improved the fabrication reproducibility of $\mathrm{MASnI}_{3}$ devices. ${ }^{40}$ The resultant devices delivered an outstanding PCE of $7.13 \%$, which is hitherto the highest among $\mathrm{MASnX}_{3}$ based PSCs.

FA-Sn-X PVSKs: Despite the successful and wide exploitation of $\mathrm{MA}^{+}$in $\mathrm{Sn}-\mathrm{PSC}$, the phase transition from tetragonal to cubic phase at $57{ }^{\circ} \mathrm{C}$, which readily occurs in practical operation conditions, appears to restrict its further development. ${ }^{41}$ The replacement of $\mathrm{MA}^{+}$with $\mathrm{FA}^{+}$was reported as one effective means to improve phase stability up to $200{ }^{\circ} \mathrm{C}$, which arises from the enhanced hydrogen bonding between $\mathrm{FA}^{+}$and the inorganic matrix. ${ }^{42}$ Moreover, Zhang and coworkers verified theoretically the weaker antibonding coupling between Sn-5s and I-5p in $\mathrm{FASnI}_{3}$ than $\mathrm{MASnI}_{3}$ due to the larger ionic size of $\mathrm{FA}^{+}$, which resulted in higher formation 
energies of $\mathrm{Sn}$ vacancies and thus better stability of $\mathrm{FASnI}_{3} \cdot{ }^{43}$ In addition to phase stability, the FA-based Sn-PVSKs possess broader optical absorption, which indicates better photovoltaic performance than MA counterparts owing to a notable increase of photocurrent. However, the initial trial of $\mathrm{FA}^{+}$based $\mathrm{Pb}$-free PSCs showed a limited PCE of $2.1 \%,{ }^{44}$ which is attributed to the poor film morphology because of uneven crystallization process. Such dilemma was resolved by Yan and coworkers who introduced diethyl ether anti-solvent to trigger simultaneous nucleation and prepared smooth film morphology as shown from the scanning electron microscope (SEM) imaging in Figure 2D-I, ${ }^{45}$ resulting in an optimal PCE of $6.22 \%$. Recently, a newly discovered additive, ethylene diammonium diiodide $\left(\mathrm{EDAI}_{2}\right)$, was employed by the Diau team, to form an adduct of $\mathrm{FASnI}_{3}-\mathrm{yEDAI}$ 列 assivate grain boundaries (GBs, Figure $\left.2 \mathrm{~J}\right){ }^{46}$ The as-fabricated PSCs exhibited a continuous passivation process with the PCE increasing from $6.25 \%$ when prepared freshly to $8.9 \%$ after storage for over $1,400 \mathrm{~h}$, which can be ascribed to the crystal strain relaxation upon the gradual effect of $\mathrm{EDAI}_{2}$. Aside from solar cell applications, the unique properties of $\mathrm{FASnI}_{3}$ render it as promising materials for other optoelectronic devices such as lasers ${ }^{47}$ and $\mathrm{PDs}^{48,49}$, which will be discussed in the later sections. 

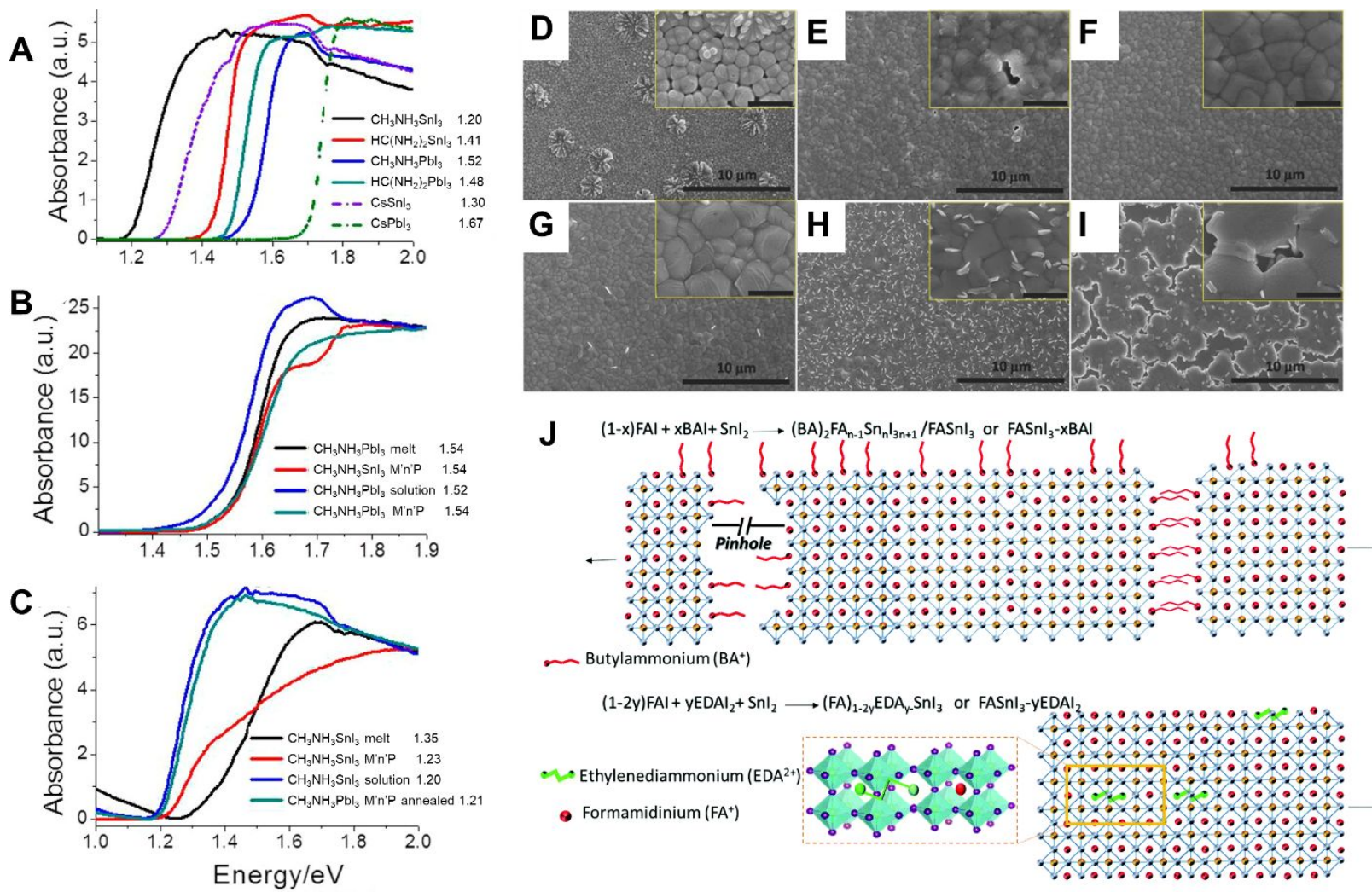

Figure 2. Absorption spectra of (A) solution-processed $\mathrm{Sn}$ and $\mathrm{Pb}$ based 3D PVSKs, as well as various (B) $\mathrm{MAPbI}_{3}$ and (C) $\mathrm{MASnI}_{3}$ perovskites depending on the synthetic methods. (A-C) Reprinted with permission from Ref. 112. Copyright 2020, American Chemical Society. SEM images of $\mathrm{FASnI}_{3} \mathrm{PVSK}$ films on PEDOT:PSS with varying concentration of $\mathrm{SnF}_{2}$ : (D) $0 \mathrm{~mol} \%$, (E) $5 \mathrm{~mol} \%$, (F) $10 \mathrm{~mol} \%$, (G) $15 \mathrm{~mol} \%$, (H) $20 \mathrm{~mol} \%$, (I) $30 \mathrm{~mol} \%$. The inset shows corresponding magnification with a scale bar of $1 \mu \mathrm{m}$. (D-I) Reprinted with permission from Ref. 45. Copyright 2019, Wiley-VCH. (J) Schematic representation of Sn-PVSK crystals in the presence of BAI and $\mathrm{EDAI}_{2}$ additives. Reproduced from Ref. 46 with permission from The Royal Society of Chemistry.

Cs-Sn-X PVSKs: The low thermal compositional stability of the above-mentioned organic cations $-\mathrm{FA}^{+}$and $\mathrm{MA}^{+}$—under high temperature remains to be a bottleneck for the applications in device. This is expected to be solved by establishing all-inorganic Cs-Sn-X type PVSKs with good thermal stability of $\mathrm{Cs}^{+}{ }^{+}{ }^{50}$ Typically, the Cs-Sn-X based PVSKs have many polymorphs depending on the oxidation state of $\mathrm{Cs}^{+}$and temperature.

When the Sn adopt the valence state of +2 , that is, in the chemical formula of $\mathrm{CsSnX}_{3}$, there exists various crystalline symmetries including cubic $(\mathrm{X}=\mathrm{Cl}, \mathrm{Br}$ and $\mathrm{I})$, tetragonal $(\mathrm{X}=\mathrm{Br}$ and $\mathrm{I})$, 
orthorhombic $(\mathrm{X}=\mathrm{I})$ and monoclinic $(\mathrm{X}=\mathrm{Cl})$ phases, which undergo thermally reversible phase transitions owing to the rotation of the $\left[\mathrm{SnX}_{6}\right]^{4-}$ octahedral as shown in Figure $3 \mathrm{~A}$ and B. ${ }^{51,52}$ During device operation, the Cs-Sn-X PVSKs suffer from unfavorable phase transition to the photo-inactive orthorhombic yellow (Y) phase, which significantly deteriorates device performance. ${ }^{51,53-55}$ Such issue was first investigated by Seshadri et al. who confirmed the competing effect of off-centering instability of $\mathrm{CsSnX}_{3}$ families caused by the $5 \mathrm{~s}^{2}$ lone pair and an emerging asymmetry in the $\mathrm{Sn}-\mathrm{X}$ correlations upon heating (Figure $3 \mathrm{C}$ and $3 \mathrm{D}$ ) ${ }^{56}$ which may be exacerbated by increasing the electronegativity of the X-site anions. ${ }^{57}$ Both works revealed the correlation between the temperature-dependent phase transitions and properties of $\mathrm{B} / \mathrm{X}$ site component. In another case, Soon and coworkers studied the impact of A-site $\mathrm{Rb}^{+}$substitution on the structural, thermodynamic and electronic nature of $\mathrm{CsSnI}_{3}$ via first-principle calculation and attributed the enhanced phase stability to the decreased free energy that was caused by increased configurational entropy. Moreover, they claimed the importance of surface termination for stabilizing Sn-based halide PVSKs, ${ }^{58}$ which was also stressed by Cai et al. ${ }^{59}$ Such theoretical assumption was experimentally confirmed by Malavasi and coworkers, corroborating the local octahedral distortion caused by increasing $\mathrm{Rb}^{+}$content, which remarkably improved the phase stability of $\mathrm{CsSnBr}_{3}{ }^{60}$

In practical applications, though the intrinsic properties of $\mathrm{CsSnX}_{3}$ such as electronic structures, ${ }^{59}$ excitonic properties ${ }^{52}$ and dielectric constant ${ }^{61}$ was extensively investigated with a predicted PCE of $\sim 23 \%,{ }^{62}$ the first attempt of $\mathrm{CsSnX}_{3}$ was not used as active layer, yet for hole conduction use in all-solid-state dye-sensitized solar cells, which implies its potential as hole transporting layer (HTL) in Sn-PSCs. ${ }^{63}$ Subsequently, Mi and colleagues revealed that $\mathrm{CsSnBr}_{3}$ exhibited not only superior intrinsic properties as a free carrier semiconductor to their organic $\mathrm{MA}^{+}$ 
and $\mathrm{FA}^{+}$based counterparts, but also remarkably high resistance towards heat induced decomposition. ${ }^{61}$ Impressively, an outstanding PCE of $7.11 \%$ in $\mathrm{CsSn}_{0.5} \mathrm{Ge}_{0.5} \mathrm{I}_{3}$ based PSCs were reported by Padture and coworkers, ${ }^{64}$ which further demonstrated the prospect of the $\mathrm{CsSnX}_{3}$.
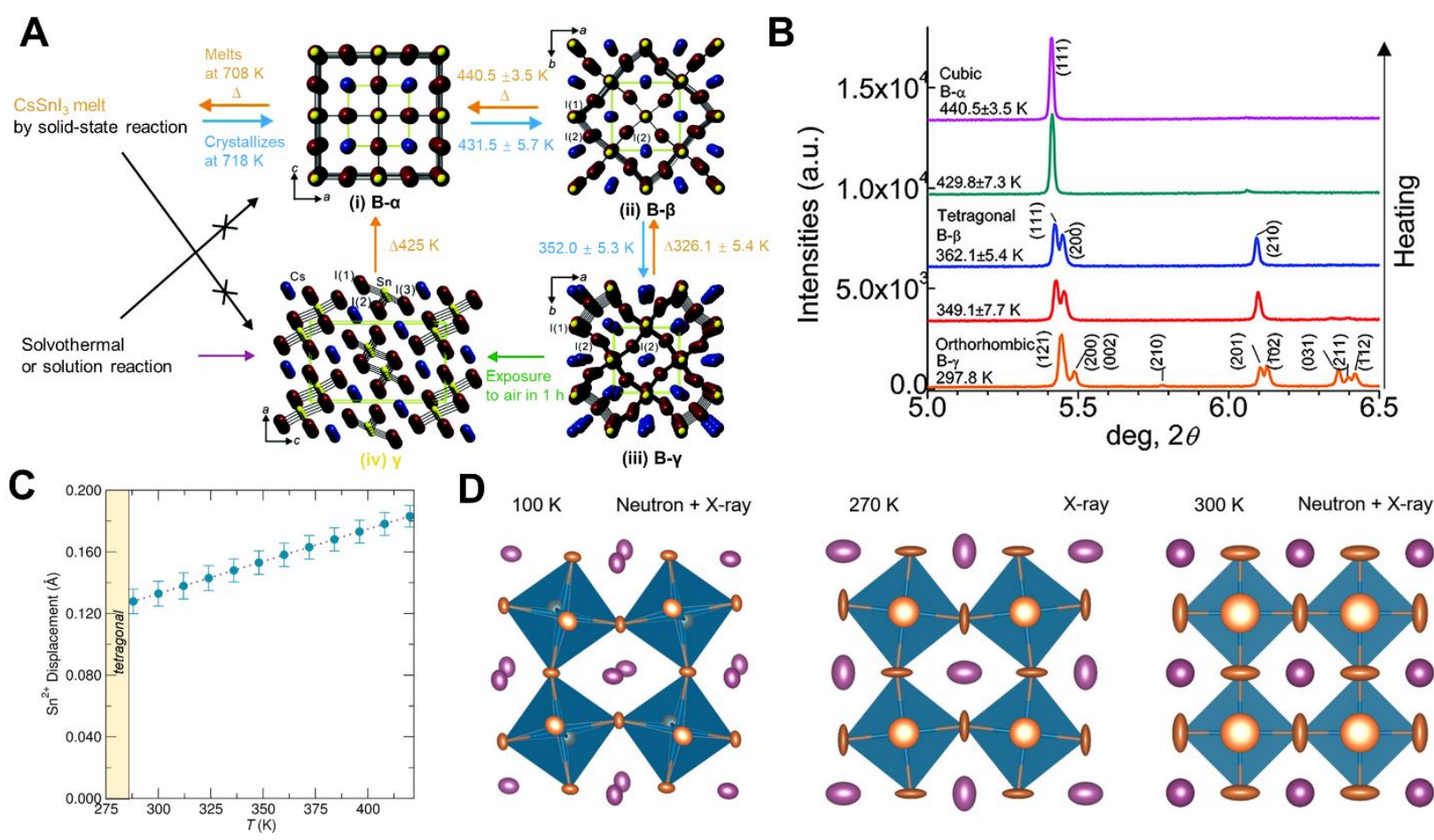

Figure 3. Structural characteristics of all-inorganic perovskites. (A) Illustration of phase transition among the four $\mathrm{CsSnI}_{3}$ polymorphs with varied synthesis conditions and conversion temperatures obtained by in situ temperature-dependent synchrotron powder XRD studies. Crystal structures of the respective polymorph are shown: (i) black cubic (B- $\alpha)$ at $500 \mathrm{~K}$, (ii) tetragonal (B- $\beta$ ) at $380 \mathrm{~K}$, (iii) orthorhombic (B- $\gamma$ ) at $300 \mathrm{~K}$ and (iv) yellow (Y) phase at $300 \mathrm{~K}$. Cs atoms, blue; Sn atoms, yellow; I atoms, violet. Green dashed lines represent a unit cell. (B) In-situ temperature-dependent synchrotron XRD patterns of black $\mathrm{CsSnI}_{3}$ on heating. (A, B) Reprinted with permission from Ref. 51. Copyright 2019, American Chemical Society. (C) Increasing $\mathrm{Sn}^{2+}$ displacement from the center of the $\left[\mathrm{SnBr}_{6}\right]^{4-}$ octahedron as temperature rises, determined from fitting the X-ray PDF over a range from 2 to $5 \AA$ against the rhombohedral R3m model. (D) Evolution of the crystallographic structures of $\mathrm{CsSnBr}_{3}$ upon heating from orthorhombic Pnma at $100 \mathrm{~K}$, tetragonal P4/mbm at 270 K, to cubic Pm3m at 300 K. (C, D) Reprinted with permission from Ref. 56. Copyright 2019, American Chemical Society.

Other A-Site Based Sn-PVSKs: Since the Sn based PVSKs is less constrained by the tolerance factors compared to its $\mathrm{Pb}$ analogues, ${ }^{65,66}$ various organic molecules beyond the tolerance factor ${ }^{67-74}$ have also been demonstrated accommodable in forming 3D PVSK networks with 
possibility of deriving novel $\mathrm{AMX}_{3}$-like structures. For instance, the Kanatzidis group reported the formation of '3D hollow' structures by substituting Sn-I units with moderate-sized diammonium cations such as ethylene diammonium $(\{\mathrm{en}\}),{ }^{71,72}$ propylene diammonium $(\mathrm{PN})$ and trimethylene diammonium $(\mathrm{TN}),{ }^{73}$ which was also demonstrated by Diau et al. with incorporation of hydroxyethylammonium into $\mathrm{FASnI}_{3} .{ }^{74}$ As a result, such $3 \mathrm{D}$-hollow structure exhibits both flexible $E_{\mathrm{g}}$ tunability and low trap densities. Importantly, the versatile $\mathrm{ASnX}_{3} \mathrm{PVSK}_{\text {s }}$ provide systematic material platforms for in-depth studies on the relationship between structural tailoring and semiconductor properties.

Low-Dimensional Sn-PVSKs. As the alkyl chain of cations becomes extremely large $(t>>$ 1), the PVSK lattices finally fail to maintain the $\mathrm{ABX}_{3}$ structure. However, some large molecules with certain groups such as amino-unit may serve as spacing ligands to afford low-dimensional PVSKs (LD-PVSKs). ${ }^{75-78}$ Generally, the LD-PVSKs are classified into 2D, 1D and 0D structures according to the electronically confined dimensionality. 2D PVSKs consist of one or several layers of octahedra sandwiched between ligands with 1D quantum confinement. In 1D PVSKs, the metal halide units are surrounded by ligands with different interconnection types - corner-sharing, edgesharing or face-sharing with electrically $2 \mathrm{D}$ confined configuration. As for $0 \mathrm{D}$ cases, the small clusters of metal halide octahedra are completely isolated with 3D exciton confinement. It is worth noting that Sn-based LD-PVSKs discussed here with dimensionality-dependent confinement effect are distinct from the nanomaterials with only nanoscale morphology such as nanosheets, nanowires and nanoparticles wherein the $\left[\mathrm{SnX}_{6}{ }^{-}\right]$units are interconnected in the manner of 3D PVSKs without electrical isolation. The long chain ligands effectively protect the lattices from external erosion, providing additional pathways to resolve the oxidation issue in Sn-PVSKs. 
Various methods have been developed to prepare high-quality and ambient-stable Sn-based LD-PVSKs with different dimensionalities from 2D to 0D, including solvent engineering, ${ }^{79}$ antioxidant addtion, ${ }^{80,81}$ epitaxial growth, ${ }^{82}$ hot-injection, ${ }^{83-85}$ and ion-exchange. ${ }^{86}$ Owing to the unique excitonic properties and enhanced stability, LD-PVSKs have been widely applied in LEDs $^{80,87}$ and PSCs. ${ }^{88,89}$ For instance, Wang et al. employed an aqueous acid-based synthetic route to prepare highly emissive and air-stable octylammonium (OCTAm) 2D based Sn-PVSKs (Figure 4A), which showed a high PL emission centered at $600 \mathrm{~nm}$ and zero overlap between their absorption and emission spectra as shown in Figure 4B. ${ }^{80}$ In another study, the Ma group prepared a novel organic metal halide hybrid with seesaw-shaped $\mathrm{SnBr}_{4}{ }^{2-}$ co-crystalized with $\mathrm{C}_{9} \mathrm{NH}_{20}{ }^{+}$, forming 0D structured $\left(\mathrm{C}_{9} \mathrm{NH}_{20}\right)_{2} \mathrm{SnBr}_{4} \cdot{ }^{87}$ The bulk crystals exhibit a broadband deep-red emission at $696 \mathrm{~nm}$ with a large Stokes shift of $332 \mathrm{~nm}$ upon photoexcitation as shown in Figure 4C and D. Note that the broadband photoluminescence (PL) with large stokes shift in PVSKs exhibits a strong dimensional dependence in both $\mathrm{Pb}$ and $\mathrm{Sn}$ based systems, which is closely correlated to the radiative emissions from the self-trapping excitons (STEs). Upon photo-excitation, the hot carriers quickly relax into free excitons (FEs), which are stabilized as STEs through electron-phonon coupling that induces localized lattice distortion. In 3D PVSKs, the FE-to-STE process is negligible due to the large potential barrier for trapping process, ${ }^{90}$ which gradually diminishes with the decreasing dimensionality. In 2D PVSKS, the STE induced broadband emissions only exist in certain structural configurations such as corrugated $(N-\mathrm{MEDA})\left[\mathrm{PbBr}_{4}\right](N-$ $\mathrm{MEDA}=\mathrm{N}^{1}$-methylethane-1,2-diammonium $)^{91}$ and $(\mathrm{EDBE})\left[\mathrm{PbBr}_{4}\right] \quad\left(\mathrm{EDBE}=2,2^{\prime}-\right.$ (ethylenedioxy)bis-(ethylammonium) $)^{3}$ and such emissions may be attributed to their different dispersion relations in the vicinity of the band bottom. ${ }^{92}$ Similarly, the barrier separating the FE and STE states almost disappears in 1D PVSKs, which leads to room-temperature observation of 
red-shifted broad emissions. The case is a bit different for OD PVSKs in which the electronic band degenerates into discrete energy levels and hence their broadened PL can be interpreted by the molecular orbit theory whereby the photon-excited structural re-organization assists in relaxing the high energy excited states into lower ones. ${ }^{77}$ By comparison between $\mathrm{Pb}$ and $\mathrm{Sn}$ based LDPVSKs, both of them share similar dimensionality-dependent STE formation processes, however, the structural flexibility induced by smaller radius of $\mathrm{Sn}^{2+}$ may facilitate the STE generation, which renders $\mathrm{Sn}$ based LD-PVSKs promising candidates for broadband white-light emission. ${ }^{93}$
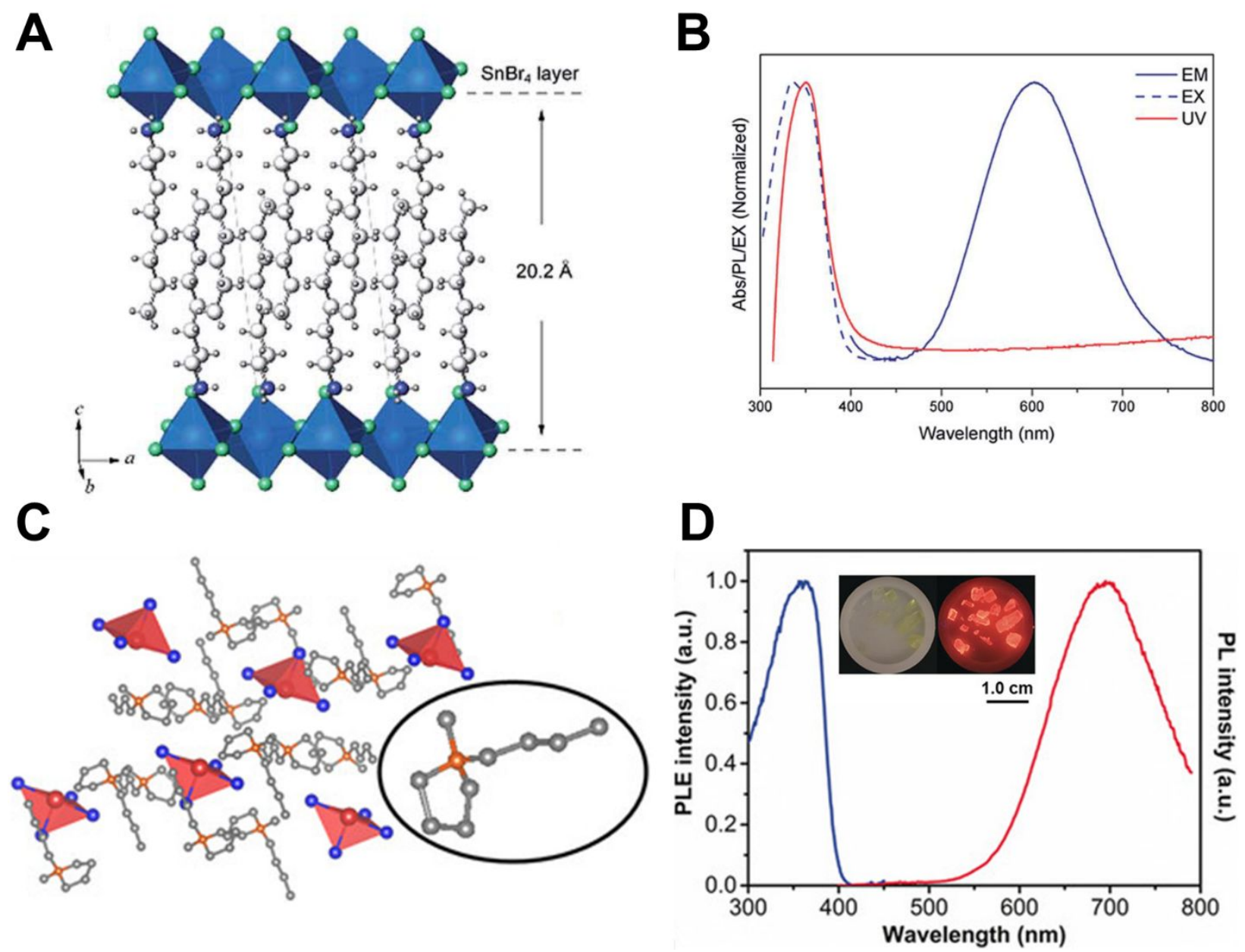

Figure 4. (A) The crystal structure of OCTAm ${ }_{2} \mathrm{SnBr}_{4}$ and (B) corresponding UV-vis absorption and PL excitation (dashed line) along with PL emission spectra (blue solid line). (A, B) Reproduced from Ref. 80 with permission from The Royal Society of Chemistry. (C) Illustration of $\left(\mathrm{C}_{9} \mathrm{NH}_{20}\right)_{2} \mathrm{SnBr}_{4}$ crystal structure, where red: $\mathrm{Sn}$, blue: $\mathrm{Br}$, orange: $\mathrm{N}$, gray: $\mathrm{C}$, red polyhedral: $\mathrm{SnBr}_{4}{ }^{2-}$. Inset: molecular structure of $\mathrm{C}_{9} \mathrm{NH}_{20}{ }^{+}$. (D) Excitation and emission spectra of $\left(\mathrm{C}_{9} \mathrm{NH}_{20}\right)_{2} \mathrm{SnBr}_{4}$ bulk crystals excited at $360 \mathrm{~nm}$. Inset in (D) images of $\left(\mathrm{C}_{9} \mathrm{NH}_{20}\right)_{2} \mathrm{SnBr}_{4}$ bulk 
crystals under ambient light (left) and UV irradiation (right). (C, D) Reprinted with permission from Ref. 87. Copyright 2019, Wiley-VCH.

Deterministic Mechanisms. The previous section presents a general view of state-of-the-art development in the Sn-based optoelectronics. In order to deepen the understanding of basic characteristics and optoelectronic properties in Sn-PVSKs and sparkle a rich variety of optimizing strategies as discussed in later sections, in this part we will concentrate on three main microscopic processes, beginning from the electronic band formation, and the subsequent photoexcitation dynamics, finally to critical issues of the degradation pathways.

Electronic Structures: Although the congener of $\mathrm{Pb}^{2+}$ and $\mathrm{Sn}^{2+}$ shared similar coordination environment, the Sn-PVSKs display notable differences in electronic structures. For instance, it was found that the ion size would significantly affect the lattice distortion and result in different $E_{\mathrm{g}}$ evolution trends. ${ }^{94}$ In general, the ionization energy and electronegativity of Pb-PVSKs are larger than Sn PVSKs. As a result, the atomic levels elevate when replacing $\mathrm{Pb}$ with $\mathrm{Sn}$ in the PVSKs with an up-shift of VBM. In addition, the splitting between $s$ and $p$ orbits in a $\mathrm{Sn}$ atom is smaller than in a $\mathrm{Pb}$ atom, which leads to the larger variation of VBM and CBM in Sn-PVSKs. The schematic energy level diagram of various Sn-based metal halide PVSKs are summarized in Figure 5A. ${ }^{95}$ Note the large Lewis acidity of $\mathrm{Sn}^{2+}$ is also the origin of fast and uncontrollable crystallization in Sn-PVSKs. ${ }^{96}$

When the $\mathrm{Pb}$ atoms are partially replaced with $\mathrm{Sn}$ in the PVSKs, the nonlinear correlation also known as the bowing relationship between $\mathrm{Sn} / \mathrm{Pb}$ ratio and the $E_{\mathrm{g}} \mathrm{s}$ can be observed. ${ }^{97-99}$ Early studies by Freeman and coworkers on $\mathrm{MASn}_{1-\mathrm{x}} \mathrm{Pb}_{\mathrm{x}} \mathrm{I}_{3}$ attributed it to the competition between the linear $E_{\mathrm{g}}$ reduction with spin-orbit coupling (SOC) and non-linear bandwidth increase caused by the lattice distortion as shown in Figure 5B. ${ }^{99}$ Since the off-centering of $\mathrm{Sn}^{2+}$ in Sn-rich phases are more pronounced than in $\mathrm{Pb}$-rich phases, the ferroelectric polarization diminishes the tilting of Sn- 
$\mathrm{I}$-Sn, resulting in negligible rise of $E_{\mathrm{g}} \mathrm{S}$ compared to the $\mathrm{SOC}$ induced decrease. Upon entering $\mathrm{Pb}$ rich regions, the non-SOC effect dominates. The widening of $E_{\mathrm{g}} \mathrm{s}$ caused by intense octahedral tilting and valence bandwidth reduction collectively compensates and/or outstrips the impact of SOC and leads to a rapid and non-linear increase of $E_{\mathrm{g}} \mathrm{s}$. On the contrary, later DFT research by Stevanović et al. excluded the impact of phase transition and stressed the main role of energy mismatch between $\mathrm{s}$ and $\mathrm{p}$ atomic orbitals of $\mathrm{Pb}$ and $\mathrm{Sn}$ on the nonlinearity. ${ }^{100}$ The bowing effect can also be observed in FA-based PVSKs. However, large FA cation would lead to the maintained band gap of $1.85 \mathrm{eV}$ till $85 \% \mathrm{Sn}$ in $\mathrm{FAPb}_{1-\mathrm{x}} \mathrm{Sn}_{\mathrm{x}} \mathrm{Br}_{3}$ with decoupled $\mathrm{SnBr}_{3}$ units. ${ }^{101}$ In addition, whereas the phase transition temperature is dominated by the $\mathrm{Sn}$ fraction in $\mathrm{Sn}-\mathrm{Pb}$ alloy PVSKs, their anomalous $E_{\mathrm{g}}$ evolution is temperature independent for regions in which all compositions do not exhibit structural disparities. ${ }^{102}$ Interestingly, when Sn is integrated with other element to form alloyed PVSKs, such anomalous $E_{\mathrm{g}} \mathrm{s}$ evolution may disappear. Nagane and colleagues reported the monotonic increased band gap with the Ge content in $\mathrm{CH}_{3} \mathrm{NH}_{3} \mathrm{Sn}_{1-\mathrm{x}} \mathrm{Ge}_{\mathrm{x}} \mathrm{I}_{3}$ PVSK, where the major contributing orbitals to the conduction band change from $\mathrm{Sn} 5 p$ orbitals to Ge $4 p$ orbitals. ${ }^{103}$ In fact, the $E_{\mathrm{gs}}$ bowing in $\mathrm{Sn}-\mathrm{Pb}$ alloyed PVSKs is not surprising among materials such as the prototypical $\mathrm{ABO}_{3}$ oxide $\mathrm{PVSKs}^{104}$ and lead chalcogenides ${ }^{105}$, which may provide model reference. In the former compounds, it was demonstrated that high-temperature phase equilibrium, compositional/structural defects and miscibility gap exhibited a remarkable influence on the bowing behavior. ${ }^{104}$ While the nonlinearity in lead chalcogenides depended largely on the solubility variation and electronegativity differences among $\mathrm{S}$, Se and Te. ${ }^{105}$ Given the complete solution and phase transition across the entire $\mathrm{Sn}-\mathrm{Pb}$ ratio range. Herein we attempt to assign $\mathrm{ASn}_{1-\mathrm{X}} \mathrm{Pb}_{\mathrm{x}} \mathrm{X}_{3}$ to the similar case of $\mathrm{BaTiO}_{3}-\mathrm{BaZrO}_{3}$ in which the bowing effect is predominately affected by the different reactivity of B-site component in the end-members as expressed: ${ }^{104}$ 


$$
E_{\mathrm{g}}(\text { excess }) \approx \mathrm{W}_{\mathrm{ASnX}_{3}} \mathrm{y}^{2}(1-\mathrm{y})+\mathrm{W}_{\mathrm{APbX}_{3} \mathrm{y}}(1-\mathrm{y})^{2}
$$

where $\mathrm{W}_{\mathrm{ASnX} 3}$ and $\mathrm{W}_{\mathrm{APbX} 3}$ represents the weighting parameters for each end-member. Mathematically, if both parameters are negative, the $E_{\mathrm{g}}$ evolution will exhibit a concave parabolic trend. Though further investigations between them remain to be explored, we suppose SOC contributes more to the $\mathrm{W}_{\mathrm{ASnX} 3}$ for its greater influence on Sn-rich phases while the non-SOC may determine $\mathrm{W}_{\mathrm{APbX} 3}$ because the steric effect takes control in $\mathrm{Pb}$-rich region based on the discussions above.

Among Sn-PVSKs, the composition of A-site cations also affects the electronic structures. In the system of $\mathrm{FA}_{1-\mathrm{x}} \mathrm{MA}_{\mathrm{x}} \mathrm{SnBr}_{3}$, the $E_{\mathrm{g}}$ value exhibited a much larger variation for $\mathrm{A}$ cation replacement (i.e., $0.5 \mathrm{eV}$ ) than $\mathrm{Pb}$ based counterparts (e.g., $0.1 \mathrm{eV}$ in $\mathrm{FA}_{1-\mathrm{x}} \mathrm{MA}_{\mathrm{x}} \mathrm{PbI}_{3} \mathrm{PVSKs}$ ). One possible origin of this new phenomenon is the contribution of MA and/or FA, especially the $p$ electrons of $\mathrm{N}$ and $\mathrm{C}$ atoms, to the density of states and in turn to the valence band characteristics. ${ }^{106}$ Besides, the influence of A cations in the spin-orbit coupling via the variation on the octahedron distortion was reported in $\mathrm{Rb}_{\mathrm{x}} \mathrm{Cs}_{1-\mathrm{x}} \mathrm{SnI}_{3}$ system. ${ }^{58}$ Surface termination of $\mathrm{Sn}$ PVSKs is as another factor modulating the electronic structures as both the ionization potentials and electron affinity are highly dependent on it. ${ }^{58}$

In particular, $\mathrm{Yu}$ and coworkers revealed the flatter band in the $\mathrm{CB}$ edge of $\mathrm{CsSnI}_{3}$ with $\mathrm{SnI}_{2}$ termination than CsI-terminations resulting in much larger electron effective masses as shown in Figure $5 \mathrm{C}$. On the other hand, the $E_{\mathrm{g}}$ itself is independent of the surface termination. ${ }^{59}$ Such a conclusion also establishes the structural difference of boundary and volume of the grains in SnPVSKs where various terminations are exposed at the GBs. Due to the homogenous $E_{\mathrm{g}} \mathrm{s}$, the energy band bending at the interface among grains could be negligible; however, the differences in electron effective masses would introduce a spatial gradient in charge carrier mobilities from the 
inside to the GBs. Besides engineering surface termination, applying an external pressure would also drastically modify the electronic structures of Sn-PVSKs. ${ }^{107,108}$ Wang and coworkers reported that the pressure induced $\left[\mathrm{SnCl}_{6}\right]^{4-}$ octahedra in $\mathrm{MASnCl}_{3} \mathrm{PVSKs}$ results in the decreasing $E_{\mathrm{g}}$ as well as strengthened interaction between $\mathrm{A}$ cations $\left[\mathrm{SnCl}_{6}\right]^{4-}$ octahedra. ${ }^{108}$ Such pressure-induced lattice distortion can trigger in situ high-pressure photoluminescence in one-dimensional Sb-based PVSK $\mathrm{C}_{4} \mathrm{~N}_{2} \mathrm{H}_{14} \mathrm{SnBr}_{4}$, which is facilitated by the lifting the activation energy of de-trapping from self-trapped states as shown in Figure 5D. ${ }^{109}$ We also noticed that some intrinsic electronic structures of 2D Sn-PVSKs have been reported. ${ }^{110,111}$ Similar to the 3D samples, Sn substitution induces a smaller bandgap and more dispersed band structure in 2D structures. ${ }^{111}$ However, whether stabilized 2D framework can diminish the oxidation of $\mathrm{Sn}^{2+}$ to $\mathrm{Sn}^{4+}$ for Sn-PVSK has yet to be rationalized. 

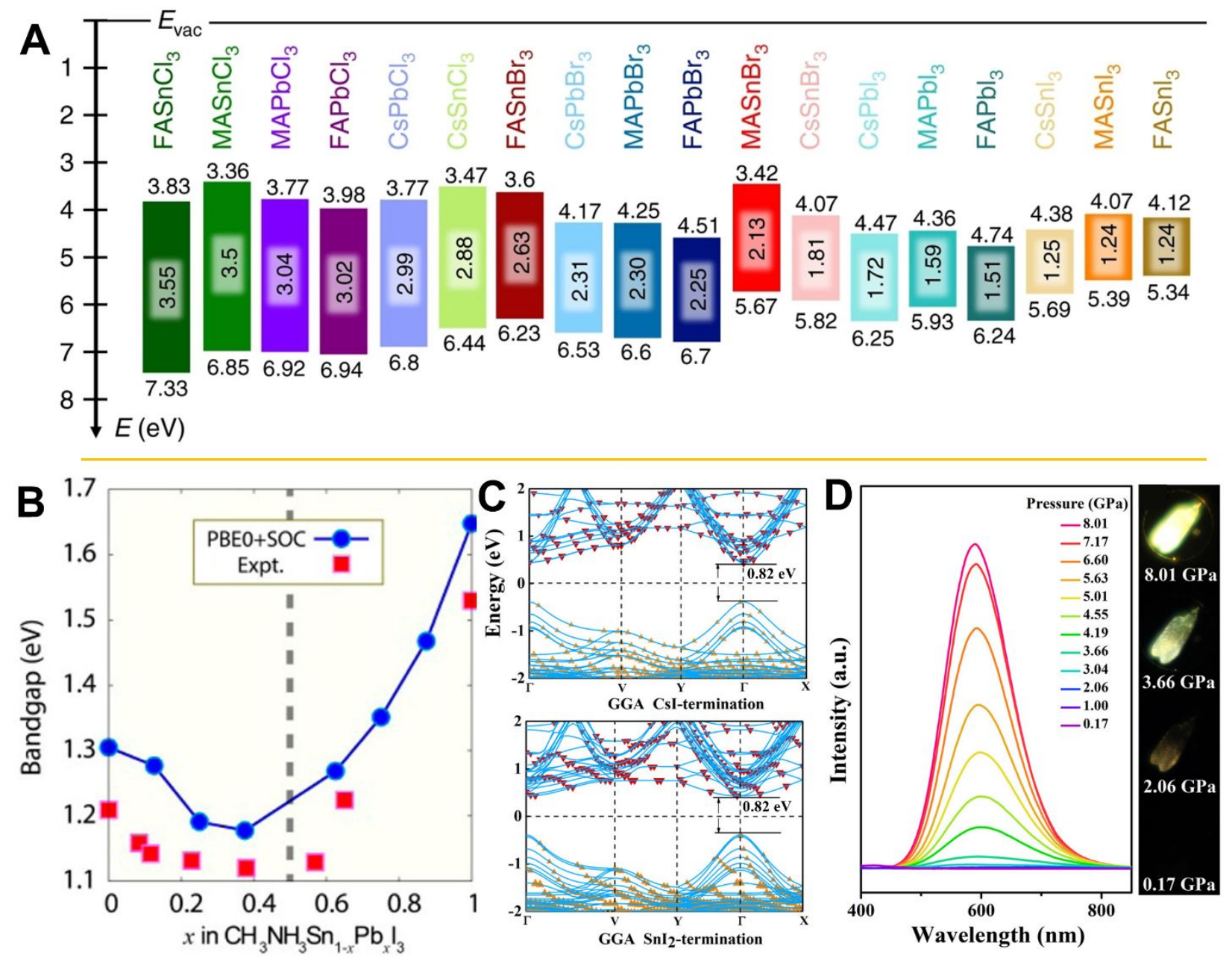

Figure 5. (A) Schematic energy level diagram of the 18 metal halide PVSKs. Reprinted with permission from Ref. 95. Copyright 2019, Springer Nature. (B) Experimental and calculated band gap evolution of $\mathrm{MASn}_{1_{-}} \mathrm{Pb}_{\mathrm{x}} \mathrm{I}_{3}$ solid solution as a function of composition $\mathrm{x}$. Reprinted with permission from Ref. 99. Copyright 2019, American Chemical Society. (C) The band structure of two different surfaces calculated by GGA-PBE. Reprinted with permission from Ref. 59. Copyright 2019, American Chemical Society. (D) Pressure-dependence of PL spectra of 2D $\mathrm{C}_{4} \mathrm{~N}_{2} \mathrm{H}_{14} \mathrm{SnBr}_{4}$. Reprinted with permission from Ref. 109. Copyright 2019, American Chemical Society.

Photophysics: In general, Sn based PVSKs exhibit high intrinsic charge carrier mobilities than $\mathrm{Pb}$ PVSKs due to larger bandwidth for both the CB and VB of the Sn-PVSK leading to the lower effective masses. ${ }^{38,112,113}$ Such an argument has been applied to different forms of Sn based PVSKs including single crystals, micro-crystals as well as bulk films. ${ }^{113}$ However, the low formation energy of Sn vacancies would inevitably result in excessive p-doping. Such a phenomenon results 
in strong monomolecular charge-carrier decay components from electron recombination with the large background hole density, which may limit the carrier diffusion and extraction as shown in Figure 6A. ${ }^{114}$ The short carrier lifetime is problematic for planar device architectures requiring a long diffusion length for carrier extraction.

The Herz team systematically investigated the influence of p-doping on the photophysics of Sn based PVSKs. ${ }^{115}$ Apart from enhanced monomolecular charge carrier recombination, a strong Burstein-Moss effect would increase absorption onset energies beyond the bandgap energy at high doping densities. In addition, the effective charge-carrier mobility would also be suppressed through scattering with ionized dopants. The large background hole density can be diminished either by engineering the A cation or employing additives specially $\mathrm{SnF}_{2}$ in the PVSKs as shown in Figure $6 \mathrm{~B}-\mathrm{D}$. The $\mathrm{SnF}_{2}$ addition would increases the $\mathrm{Sn}$ chemical potential and consequently increases the formation energy of Sn vacancy. ${ }^{38}$ In addition, $\mathrm{SnF}_{2}$ addition leads to less detrimental band alignment at interfaces and increase stability. ${ }^{116}$ Replacing $\mathrm{MA}^{+}$cations with $\mathrm{FA}^{+}$would reduce the antibonding coupling between Sn- $5 s$ and I- $5 p$ due to the larger ionic size of FA ${ }^{+}$, which is also an effective approach to the formation energies of Sn vacancies. ${ }^{43}$ The addition of Ge into the $\mathrm{FA}_{0.75} \mathrm{MA}_{0.25} \mathrm{Sn}_{1-\mathrm{x}} \mathrm{Ge}_{\mathrm{x}} \mathrm{I}_{3}$ framework was further reported by Hayase and coworkers to strikingly suppresses the trap density by order of magnitudes. ${ }^{117}$

Another critical photo-physical process determining the final performance of the PSCs is the charge transfer at the interface between PVSKs and electron transporting layer (ETL) or hole transporting layer (HTL). Earlier DFT calculation by the Zeng group revealed the potential wells between PVSK and $\mathrm{TiO}_{2}$ that could be introduced by the $\mathrm{Sn}$ addition. Such potential well is induced by the enhanced charge accumulation in $\mathrm{TiO}_{2}$ side for $\mathrm{Sn}$ rich samples due to more polar surface confirmed from the calculation as shown in Figure 6E. ${ }^{118}$ Despite the interfacial potential 
well, ultrafast spectroscopic characterization still verifies the rapid interfacial charge transfer between Sn-PVSKs and conventional ETL such as $\mathrm{TiO}_{2}(1 \mathrm{ps}) .{ }^{119}$ More importantly, a drastic deterred interfacial backwards charge carrier recombination can be observed ( $\sim 880 \mu \mathrm{s})$, which ensured the efficient interfacial charge carrier separation in Sn-PSCs.

Sn-PVSKs also exhibit unique hot carrier dynamics which is the crucial processes for the application of hot carrier solar cells. In general, the relaxation of photoexcited high energy charge carriers above the bandgap of a semiconductor downhill to the band-edge (i.e., hot carrier cooling) includes three stages, that is, i) carrier-carrier scattering leading to a quasi-equilibrium distribution of hot carrier, ii) carrier-optical phonon scattering to relax the hot carrier to the band extrema by dissipating their excess energy as heat to the lattice via phonon emission, and iii) decay of an optical phonon into acoustic phonons. The slow hot carrier cooling dynamics has long been observed in lead halides PVSKs attributed to the hot-phonon bottleneck as well as the effect of Auger re-heating effect. ${ }^{120,121}$ Very early study reveal the similarly slow hot carrier decay in lead free $\mathrm{MASnI}_{3}$ PVSKs at a timescale of $0.5 \mathrm{ps}^{38}$ Extremely long-lived hot carriers (up to a few ns) with the possibility for hot PL emission has been observed in $\mathrm{FASnI}_{3}$ later by the Loi group as demonstrated in Figure 6F. ${ }^{122}$ Such slow hot carrier cooling in Sn based PVSKs has been rationalized as i) smaller valence electronic density of states compared with $\mathrm{Pb}$ based counterparts leading to a reduction of the relaxation paths and ii) pronounced Rashba-Dresselhaus effect that reinforce the hot-phonon bottleneck. In the Pb-Sn alloyed PVSKs, a slower cooling process can be observed with the increasing of the Sn content revealed by Verma and coworkers. ${ }^{123}$ The believed the Sn addition would influence all the three stages of the cooling process. First, increasing Sn content would increase carrier density along with stronger carrier-carrier scattering. Second, Sn addition should also lead to an increased effective dielectric constant, giving rise to 
stronger screening, resulting in a suppressed rate of LO phonon emission. Finally, Sn addition should also lead to the phonon band gap opening and consequent a suppressed Klemens decay mechanism for the conversion from LO phonons to acoustic photons. ${ }^{123,124}$

There are other photo-physical behaviors in Sn-PVSKs due to the deviation in electronic structures. For example, spin-dependent photocurrent becomes more prominent upon replacing the $\mathrm{Pb}$ with $\mathrm{Sn}$ in the PVSKs when the spin states are optically operated by switching the photoexcitation from linear to circular polarization due to the weakened spin-orbital coupling in Sn-PVSKs. ${ }^{125}$ Clear ferroelectricity can be visualized in FA-based Sn-PVSKs which is strongly debated to occur in traditional $\mathrm{MAPbI}_{3}$ PVSKs. It exists, and as postulated by many authors, ferroelectric charge discontinuities can affect the domain walls and GBs. ${ }^{126}$

Photophysics of nanostructured Sn based PVSKs such as nanoplatetes or nanoparticles have been investigated in the literature mostly focused on all inorganic $\mathrm{CsSnX}_{3} \cdot{ }^{83,86}$ The strong quantum confinement of those nanostructure induced clear increased optical band gap analogous to other semiconductors. ${ }^{83}$ However, the dominant intrinsic defects in $\mathrm{CsSnX}_{3}$ tended to form shallow trap states which serves as radiative recombination centers instead of quenchers for the photoluminescence. 

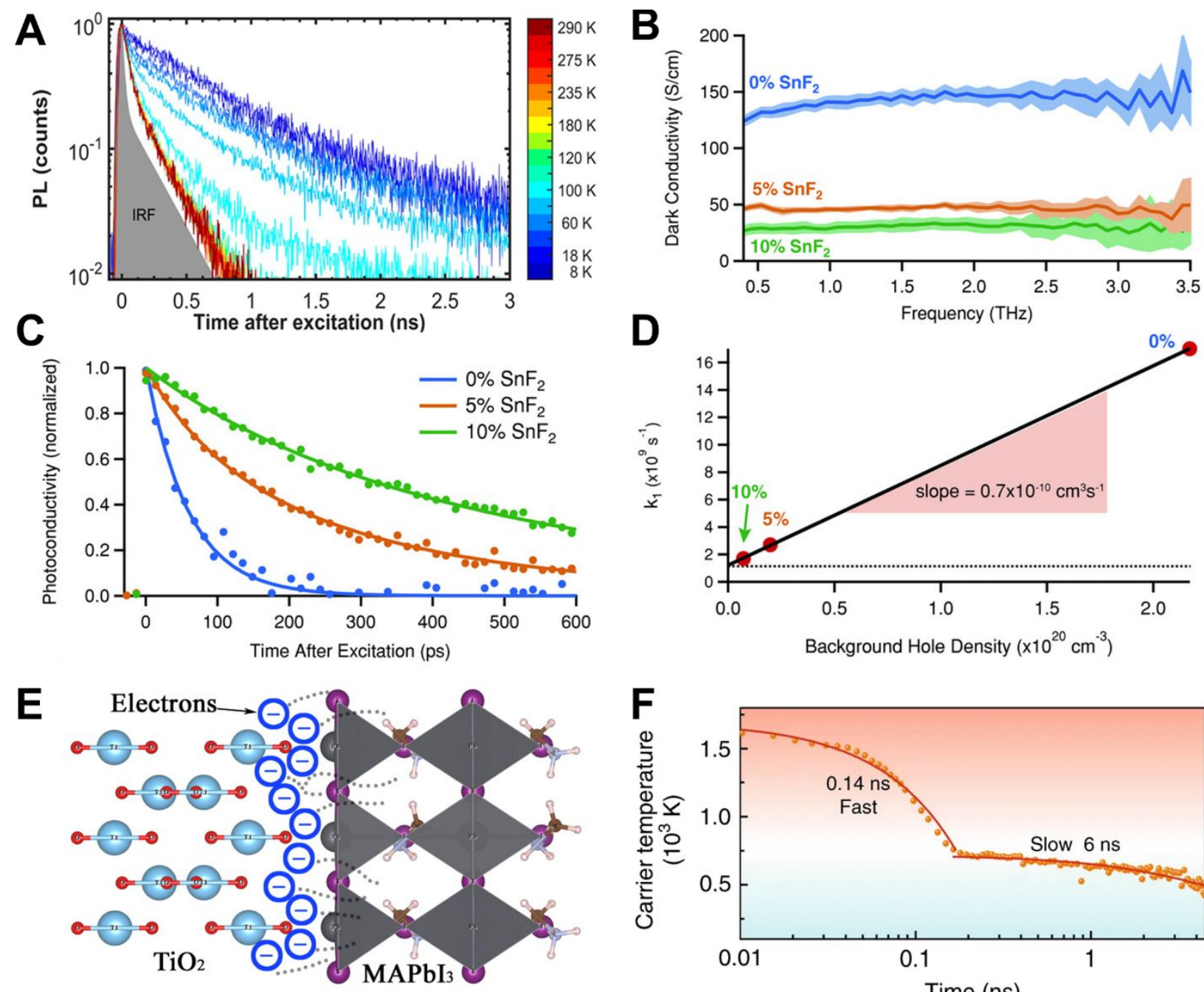

D
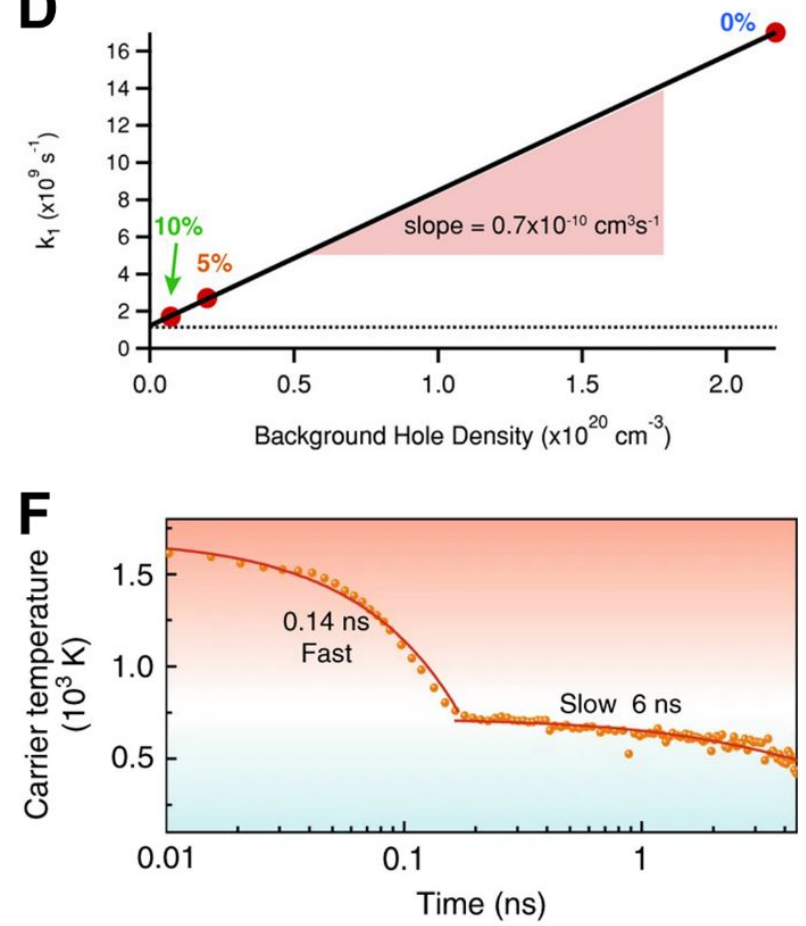

Figure 6. (A) TRPL kinetics of $\mathrm{MASnI}_{3}$ film at various temperature. Reprinted with permission from Ref. 114. https://pubs.acs.org/doi/10.1021/acs.jpclett.6b00322. Copyright 2019, American Chemical Society. (B) Diminished dark conductivity with $\mathrm{SnF}_{2}$ addition in $\mathrm{FASnI}_{3}$ film extracted from time-resolved THz (THz-TDS) spectroscopy, (C) Optical pump THz probe spectroscopy (OPTPS) measurements of the charge-carrier recombination dynamics of $\mathrm{FASnI}_{3}$ films with different concentrations of added $\mathrm{SnF}_{2}$. (D) Relationship between doping density extracted from dark conductivity spectra shown in (B), and the monomolecular charge-carrier recombination rate, $\mathrm{k}_{1}$. The red markers are experimental data, and the solid black line is a linear fit. The dashed line indicates the y-intercept, and the shaded region marks the slope of the line. (E) Schematics to show the accumulation of charges at the polar interface between PVSKs and $\mathrm{TiO}_{2}$. Reprinted with permission from Ref. 118. Copyright 2019, American Chemical Society. (F) ultra-slow hot carrier cooling dynamics with slow carrier temperature decay in $\mathrm{FASnI}_{3}$ films.

Among the applications of emitters such as LEDs or lasing devices, the basic photophysical requirements for Sn-PVSKs is identical to those of Pb-PVSKs where the non-radiative 
recombination and the trapping process should be minimized. Nonetheless, in order to obtain the high emission quantum yield, a localization of the photo-generated charge carriers in the active layer is highly preferable. Therefore, low-dimensional quantum confined systems-e.g., 2D Ruddlesden-Popper (RP) structures or quantum dots—seem to be more popular than conventional 3D PVSKs for Sn based LEDs. ${ }^{80,127-129}$ In those structures, the excitonic recombination should be dominated after charge carrier injection which is different than solar cell devices. As a result, the radiative recombination time is much shorter than in 3D Sn-PSVKs. ${ }^{127}$ Moreover, the Sn addition in the PVSK lattice was reported to enhance the above-mentioned STE formation. Such STE formation is initiated by the hole trapping at the $\mathrm{Sn}$ sites followed by the electron localization by Columbic interaction. This would induce ultra-board band PL emission for white-light LED application. ${ }^{128}$ Furthermore, the enhanced radiative monomolecular charge recombination pathway deriving from the doping can drastically facilitate the amplified spontaneous emission (ASE), and thus opening up a path for ultralow light-emission thresholds. ${ }^{47}$ The ASE chargecarrier density threshold can be found as low as $8 \times 10^{17} \mathrm{~cm}^{-3}$ in the benchmark $\mathrm{FASnI}_{3} \cdot{ }^{47}$ Besides, Sn-PVSKs exhibited 1-2 orders of magnitude larger free-electron-hole bimolecular recombination rates compared with conventional $\mathrm{MAPbI}_{3}$ after trap passivation by $\mathrm{SnF}_{2}$. This renders it promising optical gain in the laser device applications. ${ }^{128}$ However, the origins of the above superior charge carrier dynamics parameters in Sn-PVSKs have yet to be explored when comparing with $\mathrm{Pb}$ counterparts.

Atmospheric Degradation: The atmospheric instability of Sn based PVSKs has long been considered as one fatal obstacle restricting their commercialized application. Given the analogous structures between $\mathrm{Pb}$ and $\mathrm{Sn}$ based PVSKs, the degradation pathways of $\mathrm{Pb}$-PVSKs such as water and oxygen-light induced decomposition may also exist in Sn-PVSKs. In addition, Sn-PVSKs 
suffer from unwanted oxidation of $\mathrm{Sn}^{2+}$, which causes severe self-doping and destroys their semiconducting nature. For water ingression, it was demonstrated that the vacancy-type defects at the GBs played a critical role in the degradation circle of $\mathrm{MAPbI}_{3}{ }^{130}$ while the $\mathrm{PbI}_{2}$-terminated interface shows a stronger resistance to moisture thanks to the higher activation energy of water diffusion compared to MAI termination. ${ }^{131}$ It was further revealed that the $\mathrm{Pb}$ vacancies at the GBs accelerated this process. ${ }^{132}$ In addition, the light-oxygen induced decomposition is also an major degradation path in $\mathrm{MAPbI}_{3}$ by following the procedures as shown below: ${ }^{133}$

$$
\begin{gathered}
\mathrm{MAPbI}_{3} \stackrel{\text { Light }}{\longrightarrow} \mathrm{MAPbI}_{3}{ }^{*}(1) \\
\mathrm{O}_{2} \stackrel{\mathrm{MAPbI}_{3}{ }^{*}}{\longrightarrow} \mathrm{O}_{2}-(2) \\
\mathrm{MAPbI}_{3}{ }^{*}+\mathrm{O}_{2}-\stackrel{\text { deprotonation }}{\longrightarrow} \mathrm{PbI}_{2}+\frac{1}{2} \mathrm{I}_{2}+\mathrm{H}_{2} \mathrm{O}+\mathrm{CH}_{3} \mathrm{NH}_{2}
\end{gathered}
$$

Such photolysis is originated from the deprotonation (3) between the superoxide $\left(\mathrm{O}_{2}^{-}\right)$ generated upon photoexcitation ((1) and (2)) and the excited $\mathrm{MAPbI}_{3}{ }^{*}$, of which the reaction rate exhibits strong correlation with I vacancies at surfaces and GBs. When it comes to Sn PVSKs, the interfacial passivation of $\mathrm{B}$ and $\mathrm{X}$ sites related defects with tin halide additives has demonstrated effective in improving long-term ambient stability Sn-based devices, ${ }^{134-136}$ which corroborates the similar degradation processes as $\mathrm{Pb}$. For the oxidation sensitivity of $\mathrm{Sn}^{2+}$, it is an inevitable process in Sn-PVSKs regardless of light/dark conditions for the thermodynamically inferior stability of $\mathrm{Sn}^{2+}$ to $\mathrm{Sn}^{4+}$ as discussed above. Analogous to Pb-PVSKs, such a process has also been found to correlate with $\mathrm{Sn}-\mathrm{I}$ bonding. Recently, both the Hu and Nakamura groups theoretically simulated the detailed degradation processes of Sn based PVSKs and confirmed the critical role of surface Sn-I bonding on the degradation process, which would be significantly weakened by $\mathrm{H}_{2} \mathrm{O}$ or $\mathrm{O}_{2}$ invasion along with the depletion of a $\mathrm{CH}_{3}-\mathrm{NH}_{3}{ }^{+}$cation in the surface region. ${ }^{39,137}$ Such instability 
originating from the fragile surface Sn-I bonding can be well visualize from the ab initio molecular dynamics calculation as shown in Figure 7A. ${ }^{137}$ More detailed $\mathrm{Sn}^{2+}$ oxidation pathways associated with the Sn-I bond breaking in full $\mathrm{ASnI}_{3}$ PVSKs is later clarified by McGehee et al. using in-situ thermogravimetric analysis (TGA) as follows: ${ }^{138}$

$$
2 \mathrm{ASnI}_{3}+\mathrm{O}_{2} \rightarrow 2 \mathrm{AI}+\mathrm{SnO}_{2}+\mathrm{SnI}_{4}
$$

Similar degradation by-products in various Sn-PVSK systems were also validated by Stranks et al. by using the ${ }^{119} \mathrm{Sn}$ solid-state NMR spectra. ${ }^{139}$ Nishikubo and coworkers revealed the influence of such degradation on the electronic structures and charge carrier dynamics of the $\mathrm{MASnI}_{3}$ and $\mathrm{FASnI}_{3} \mathrm{PVSKs}^{140}{ }^{14}$ The degradation process down-shifted the VBM of the samples as well as declined the photoconductivity of the samples observed from time-resolved microwave conductivity measurement (TRMC) as depicted in Figure 7 B. ${ }^{140}$ They also confirmed the degradation process accompany with the formation of $\mathrm{ASnI}_{6}$ low dimensional PVSKs. Integrating $\mathrm{Pb}$ element in Sn based PVSK or shrinking the cubic lattice by incorporation of smaller A-site cations such as $\mathrm{Cs}$ has been proved to effectively diminish the $\mathrm{Sn}^{2+}$ oxidation. ${ }^{138}$ In the former case, the $\mathrm{SnI}_{4}$ formation is apparently prevented by alloying $\mathrm{Sn}$ and $\mathrm{Pb}$, forcing the oxidation reaction to proceed through the less favorable pathway, requiring more $\mathrm{Sn}-\mathrm{I}$ and $\mathrm{Pb}-\mathrm{I}$ bonds to break to form $\mathrm{I}_{2}$. In addition, either $\mathrm{SnF}_{4}$ or $\mathrm{SnCl}_{4}$ doping is another widely-accepted method to enhance the stability of Sn based PVSKs. ${ }^{140}$ Finally, the stability of the Sn-PVSKs is strongly related to the charge transport layer utilized in the device configuration as the oxidation process has been proved to be modulated by the density of microscopic pinholes and GBs among the crystallites. ${ }^{242} \mathrm{~A}$ judicious selection of hole transport layers would therefore greatly influence the overall stability of the devices. 

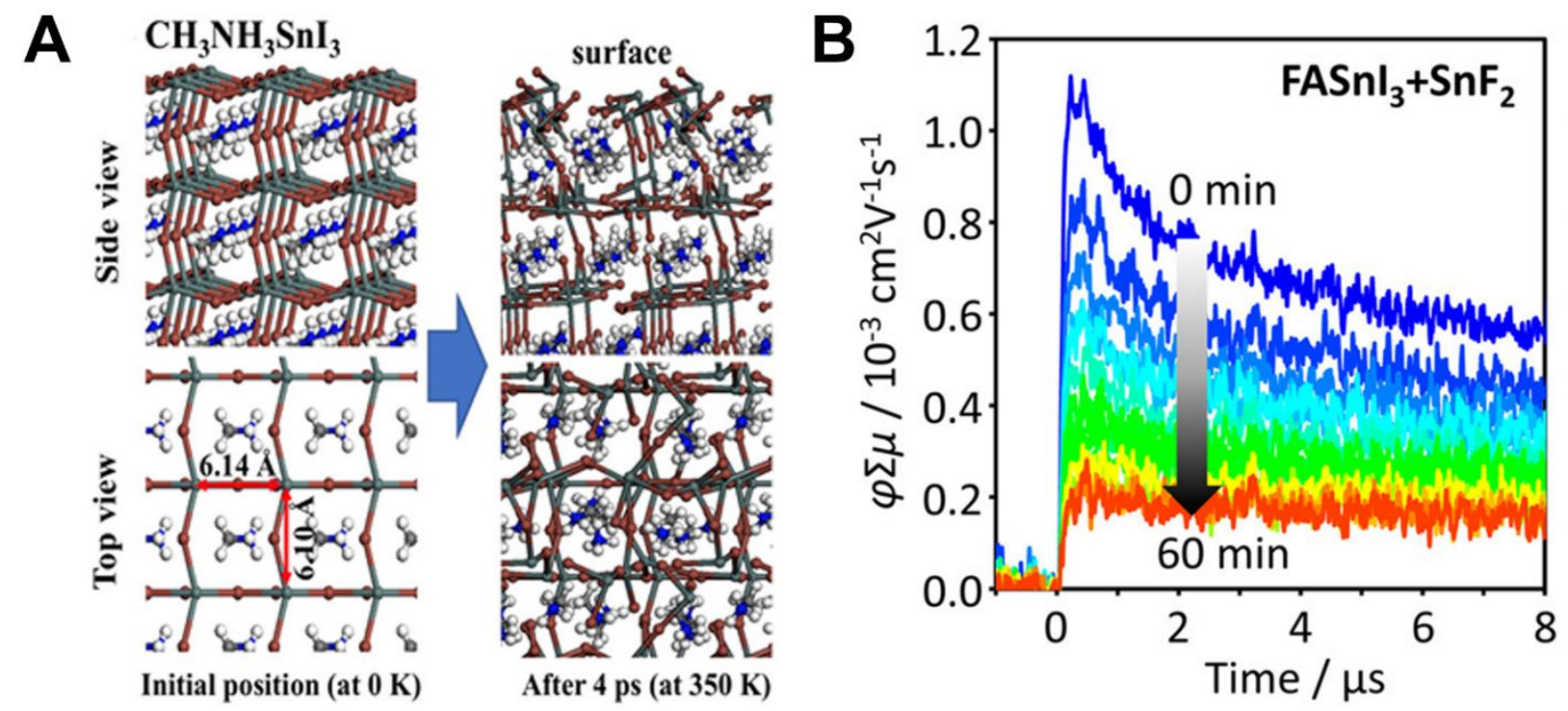

Figure 7. (A) Results of ab initio molecular dynamics calculation of $\mathrm{CH}_{3} \mathrm{NH}_{3} \mathrm{SnI}_{3}$ showing the breaking of surface Sn-I bonding. Reprinted with permission from Ref. 137. Copyright 2019, American Chemical Society. (B) TRMC transients of $\mathrm{FASnI}_{3}$ over air exposure illustrating the decreased photoconductivity with degradation. Reprinted with permission from Ref. 140. Copyright 2019, American Chemical Society.

When delving into the fundamentals of electronic structure, photophysics, and degradation pathways as elucidated above, the intrinsic issues in Sn-PVSKs emerge as 1) easy oxidation of $\mathrm{Sn}^{2+}$, which impairs long-term structural stability; 2) oxidation induced p-doping, which causes severe monomolecular recombination and $E_{\mathrm{g}}$ widening; 3) mismatched band alignment between the active layer and the transporting layers, which hinders the collection of free carriers; 4) rapid and uncontrollable crystallization, which present a critical technical challenge towards highquality preparation of Sn-PVSK thin films. However, the anomalous band bowing effect upon $\mathrm{Sn}-\mathrm{Pb}$ alloying, tunable band structure with $\mathrm{A} / \mathrm{B} / \mathrm{X}$ site ions and similar degradation pathways as $\mathrm{Pb}$ analogues may inspire enormous strategies for further enhancement in Sn-PVSKs based optoelectronics. In the next section, we will dive into detailed optimizing strategies for different Sn-PVSKs based optoelectronic devices from the aspects of additives/solvent selection, preparation improvement, compositional modulation, interfacial engineering and phase regulation. 
Photovoltaic Cells. Additives: The commonly applied additives were focused on tailoring thin-film forming process, stabilizing the Sn-PVSK structure and suppressing the oxidation of $\mathrm{Sn}^{2+}$. Based on separate functions, we divide the commonly-used additives into four categories: i) tin halide (e.g., $\mathrm{SnF}_{2}$ ), ii) Lewis base with active lone pair electrons such as thionyl or thiocyanate group), iii) antioxidant and iv) other additives. The detailed device performance and stability parameters are summarized in Table 1.

Table 1. Summary of device performance and stability of Sn-PSCs by the additive strategy

\begin{tabular}{|c|c|c|c|c|c|c|c|}
\hline Active layer & Strategy & $\begin{array}{l}V_{\text {OC }} \\
(\mathrm{V})\end{array}$ & $\begin{array}{c}J_{\mathrm{SC}} \\
\left(\mathbf{m A} / \mathbf{c m}^{2}\right)\end{array}$ & $\begin{array}{l}\text { FF } \\
(\%)\end{array}$ & $\begin{array}{l}\text { PCE } \\
(\%)\end{array}$ & $\begin{array}{l}\text { Stability test } \\
\text { (r.t.) }\end{array}$ & Ref. \\
\hline $\mathrm{FASnI}_{3}$ & $\mathrm{SnF}_{2}$ & 0.24 & 24.4 & 36 & 2.1 & N/A & 44 \\
\hline $\mathrm{CsSnBr}_{3}$ & $\mathrm{SnF}_{2}$ & 0.41 & 9.1 & 57 & 2.2 & $\begin{array}{c}\text { MPPT, } 65 \% \text { RH, } 15 \\
\min (0 \%)\end{array}$ & 116 \\
\hline $\mathrm{CsSnI}_{3}$ & $\mathrm{SnCl}_{2}$ & 0.50 & 9.9 & 68 & 3.6 & $\begin{array}{l}\text { Continuous operation, } \\
25 \% \mathrm{RH}, 16 \mathrm{~h}(70 \%)\end{array}$ & 134 \\
\hline $\mathrm{CsSnI}_{3}$ & $\mathrm{SnBr}_{2}$ & 0.44 & 18.5 & 53 & 4.3 & Shelf life, $\mathrm{N}_{2}, 100 \mathrm{~h}$ & 135 \\
\hline $\mathrm{CsSnI}_{3}$ & $\mathrm{SnF}_{2}$ & 0.24 & 22.7 & 37 & 2.0 & $\begin{array}{c}\text { Shelf life, } \mathrm{N}_{2}, 250 \mathrm{~h} \\
(100 \%)\end{array}$ & 142 \\
\hline $\mathrm{CsSnI}_{3}$ & $\mathrm{SnI}_{2}$ & 0.43 & 12.3 & 40 & 2.8 & $\begin{array}{c}\text { Shelf life, } \mathrm{N}_{2}, 100 \mathrm{~d} \\
(60 \%)\end{array}$ & 213 \\
\hline $\mathrm{FASnI}_{3}$ & $\mathrm{EDAI}_{2}$ & 0.58 & 21.3 & 72 & 8.9 & $\begin{array}{c}\text { Shelf life, } \mathrm{N}_{2}, \\
1500-2000 \mathrm{~h}(100 \%)\end{array}$ & 46 \\
\hline $\mathrm{CsSnI}_{3}$ & $\begin{array}{l}\text { Piperazin } \\
\text { e } \\
\& \mathrm{SnCl}_{2}\end{array}$ & 0.34 & 20.6 & 54 & 3.8 & $\begin{array}{c}\text { Continuous operation, } \\
\text { 40-50\% RH, } 5 \mathrm{~min} \\
(80 \%)\end{array}$ & 143 \\
\hline $\mathrm{FASnI}_{3}$ & Pyrazine & 0.32 & 23.7 & 63 & 4.8 & $\begin{array}{c}\text { Shelf life, } \\
\text { encapsulated, } 100 \mathrm{~d} \\
(98 \%)\end{array}$ & 147 \\
\hline $\begin{array}{c}\mathrm{FA}_{0.7} \mathrm{Cs}_{0.3} \mathrm{~Pb}_{0 .} \\
{ }_{7} \mathrm{Sn}_{0.3} \mathrm{I}_{3}\end{array}$ & $\begin{array}{l}\mathrm{FACl} \& \\
\mathrm{SnF}_{2}\end{array}$ & 0.74 & 25.9 & 81 & 15.8 & $\begin{array}{c}\text { Shelf life, } 20 \% \text { RH, } \\
288 \text { h }(90 \%)\end{array}$ & 149 \\
\hline
\end{tabular}


Note: a) Abbreviation: MPPT = max power point tracking, r.t. $=$ room temperature, $\mathrm{RH}=$ relative humidity, Ref. = reference, $\mathrm{KHQSA}=$ hydroquinone sulfonic acid, $\mathrm{EDAI}_{2}=$ ethylene diammonium diiodide, TPPi $=$ triphenyl phosphite, $\mathrm{HZ}=$ hydrazine, AA $=$ ascorbic acid, 5-AVAI = 5ammonium valeric acid iodide; b) Terms for stability test, 'Test condition, Storage condition, Duration (Remnant PCE percentage)', for instance, the 'MPPT, 65\% RH, 15 min (0\%)' means the device was measured under air ambient with $65 \% \mathrm{RH}$ under room temperature. The test condition is MPPT and the device kept $0 \%$ of the initial PCE after 15 min of measurements.

For tin halide additives, $\mathrm{SnF}_{2}$ was first introduced by the Mathew group into $\mathrm{CsSnI}_{3}$ system, which rendered it lower intrinsic conductivity owing to the increase in the formation energy of Sn vacancy as shown in Figure $8 \mathrm{~A}$ and resulted in higher carrier densities as shown in Figure $8 \mathrm{~B} .{ }^{142}$ The same effect can be found for $\mathrm{FASnI}_{3}$ with an encouraging PCE of $2.10 \%$ in a normal device structure, triggering the subsequent enormous development of tin halide PVSKs assisted by $\mathrm{SnX}_{2}$ ( $\mathrm{X}=\mathrm{F}, \mathrm{Cl}, \mathrm{Br}, \mathrm{I})$, which is now regarded as paradigm additives for synthesis of lead-free halide 
PVSKs for optoelectronics. ${ }^{44,45,116,134-136,143-146}$ Intriguingly, $\mathrm{SnX}_{2}$ additives were reported to take into effect in different ways depending on the halogen atoms. For example, slightly excessive $\mathrm{SnI}_{2}$ addition was reported to stabilize the PVSKs lattices by compensating the $\mathrm{Sn}^{2+}$ loss upon oxidation as displayed in Figure 8C. ${ }^{146}$ While $\mathrm{SnCl}_{2}$ was found to serve as desiccants and slow down the oxidation process. ${ }^{134,143}$ Additionally, Im et al. demonstrated $\mathrm{SnBr}_{2}$ as both effective surface passivator and stabilizer of the B- $\gamma$ black phase of $\mathrm{CsSnI}_{3}$ because of its lowest adsorption energy and strongest binding on the surface among the three kinds of tin halide additives. ${ }^{135}$ In short, $\operatorname{SnX} 2$ is believed to not only ensure the $\mathrm{Sn}^{2+}$-rich environment, increase the formation energy of $\mathrm{Sn}$ vacancies and thus suppresses the self-doping effect, but also improve its phase stability. Besides, $\mathrm{SnX}_{2}$ may still be utilized to optimize the interfacial band alignment for separation of photoinduced excitons, ${ }^{116,134}$ which is of great significance in reducing the $V_{\mathrm{OC}}$ loss and improving the FF in device.

Second, some Lewis acids and bases theory can effectively stabilize the frame structure of $\mathrm{Pb}-$ PVSKs through the interaction between the lone pair electrons of Lewis base and $\mathrm{Pb}^{2+}$. Likewise, the strategy of $\mathrm{SnX}_{2}$ addtive Lewis-adduct had also proved valid for Sn-PVSKs. ${ }^{46,143,147-154}$ For example, the sulfonate group were reported to interact with $\mathrm{Sn}^{2+}$ in both $\mathrm{Sn}-\mathrm{PVSK}$ s and the excessive $\mathrm{SnCl}_{2}$ additives (Figure 8D), which not only regulate the crystallization of precursor solution, but also protect the polycrystals through in-situ encapsulation by $\mathrm{SnCl}_{-} \mathrm{SO}_{3}{ }^{-}$adducts at GBs. ${ }^{150}$ Later, pseudo-halide additives have been confirmed to coordinate with $\mathrm{Sn}^{2+}$ and passivate the interfacial trap states. ${ }^{19,88,149-153}$ The Oh group initiated the application of the FASCN into FA based PVSKs, which was confirmed by XPS to interact with $\mathrm{Sn}^{2+}$ to prevent the oxidation during crystallization and enhance the crystallinity of the films. ${ }^{151}$ Such SCN based Lewis base was used by Ning's group to separate the nucleation and growth processes in 2D Sn-PVSKs with aid of the 
removable $\mathrm{NH}_{4} \mathrm{SCN}$ and delivered a record PCE of $9.41 \%{ }^{88}$ Aside from direct addition to precursor, these additives were recently combined with anti-solvents by several research teams to modulate film formation through Lewis acid-base interaction, ${ }^{155-157}$ which holds promising prospects. 

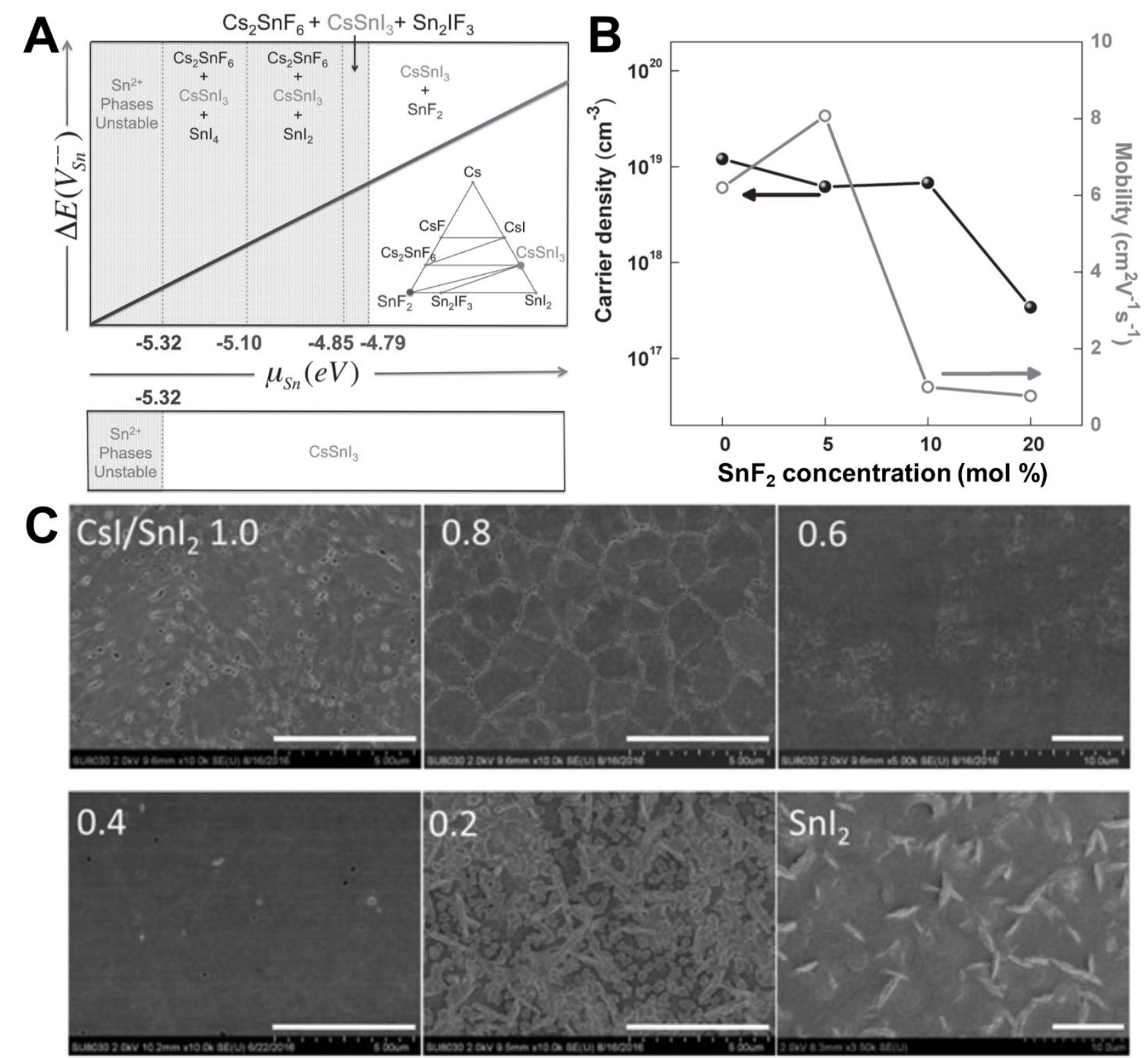

D
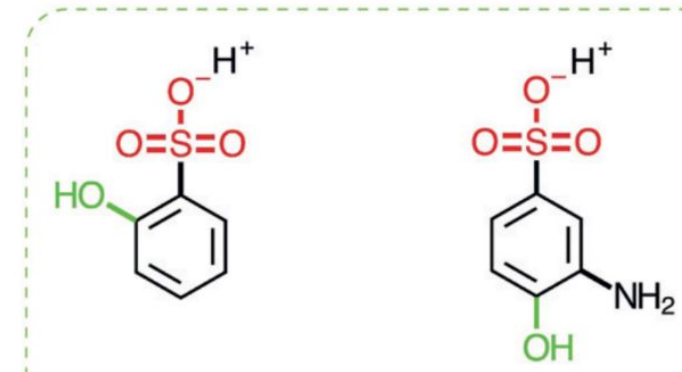

PSA

APSA
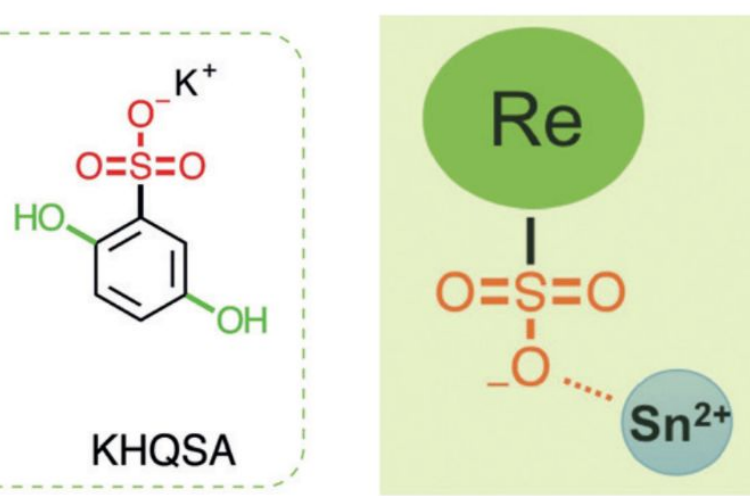

Figure 8. (A) Theoretical evolution between the Sn vacancy formation energy $\left(\Delta \mathrm{E}\left(\mathrm{V}_{\mathrm{Sn}}\right)\right)$ and $\mathrm{Sn}$ chemical potential $\left(\mu_{\mathrm{Sn}}\right)$. (B) Carrier concentration and mobility of $\mathrm{CsSnI}_{3}$ PVSK films with 
various $\mathrm{SnF}_{2}$ addition. (A, B) Reprinted with permission from Ref. 142. Copyright 2019, WileyVCH. (C) SEM images of the $\mathrm{CsSnI}_{3}$ PVSK films grown with different $\mathrm{CsI} / \mathrm{SnI}_{2}$ molar ratios. Reprinted with permission from Ref. 136. Copyright 2019, American Chemical Society. (D) Molecular structures of phenol sulfonic acid (PSA), 2-aminophenol-4-sulfonic acid (APSA) and hydroquinone sulfonic acid (KHQSA) and proposed interaction between the additive and $\mathrm{Sn}^{2+}$. Reprinted with permission from Ref. 150. Copyright 2019, Wiley-VCH.

Third, the antioxidant additives have been applied to hinder the oxidation in Sn-PVSKs. ${ }^{80,81,89,}$ 136,158-165 Although we should emphasize the removability of additives, which may introduce impurities and defects in as-prepared Sn-PVSK thin films, the residual additives also ensure the long-time inhibition of oxidation. Generally, the difficulty of removing supplements increases from gas, liquid to solid state, depending on which we categorize the present antioxidants into three types. The gaseous antioxidants seem limited, in which the hydrazine vapor (HZ) was mostly exploited to create a reducing atmosphere during preparation of $\mathrm{MASnI}_{3}$ as shown in Figure 9A. ${ }^{158}$ On the other hand, The solid-state antioxidants comprise both elemental substance. ${ }^{159,160,163}$ and compounds. ${ }^{164,165}$ The metal powder of Sn was employed to directly react with the Sn-PVSK precursor solution, thus maintaining $\mathrm{Sn}^{2+}$-rich environment by comproportionating process between $\mathrm{Sn}$ and $\mathrm{Sn}^{4+}$. Figure 9B shows the direct solid-liquid com-proportionation between $\mathrm{Sn}$ powder and $\mathrm{MASnI}_{3}$ solution under continuous stirring. Using this method, Tan and coworkers achieved an impressive certified PCE of $19.5 \%$ and the resulting all-PVSK tandem solar cells delivered an outstanding stability of $463 \mathrm{~h}$ under maximum power point tracking (MPPT) in inert atmosphere. For the aqueous antioxidants, it was found that triphenyl phosphine (TPPi) addition effectively enhanced device durability of $\mathrm{CsSnI}_{3}$ QD PSCs (Figure 9C), which resulted in stable operation for 25 days in an inert atmosphere. ${ }^{89}$

Besides, other kinds of additives such as $\mathrm{C}_{60},{ }^{166,169}$ 5-ammonium valeric acid iodide (5AVAI) ${ }^{167}$ and poly(vinyl alcohol) (PVA) ${ }^{168}$ have manifested to interact with Sn-PVSK lattices via other forces such as hydrogen bonds, intermolecular forces and halide bonding. For example, the 
Islam group succeeded to passivate GBs of $\mathrm{FASnI}_{3}$ by 5-AVAI, which formed hydrogen bonds with $\mathrm{I}^{-}$with the carboxylic acid and the ammonium end-groups. Thus, the devices exhibited enhanced durability than pristine $\mathrm{FASnI}_{3}$ based devices by maintaining $75 \%$ of the initial PCE after maximum power point tracking for $100 \mathrm{~h}$ in ambient atmosphere. ${ }^{167}$ Moreover, the fullerene and its derivatives were reported to passivate GBs. ${ }^{169}$ After spin-coating, $\mathrm{C}_{60}$ will disperse uniformly at the GBs and efficiently prevented the intrusion of oxygen and moisture into the lattices. ${ }^{166}$ 

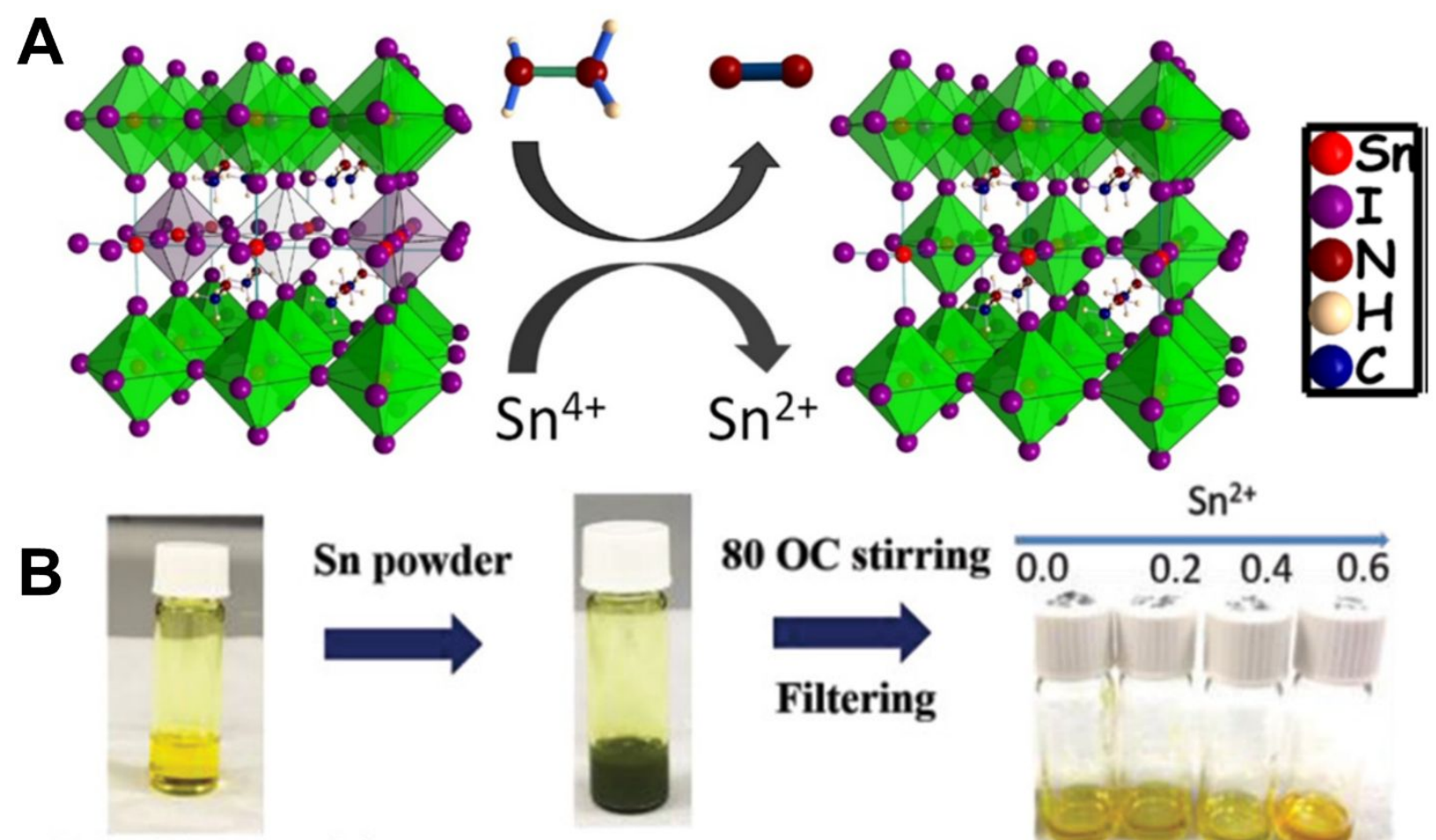

Filtering

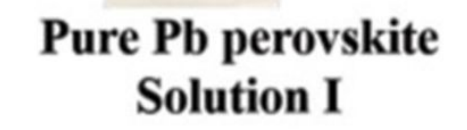

Solution II

$\mathrm{Pb}^{2+}$
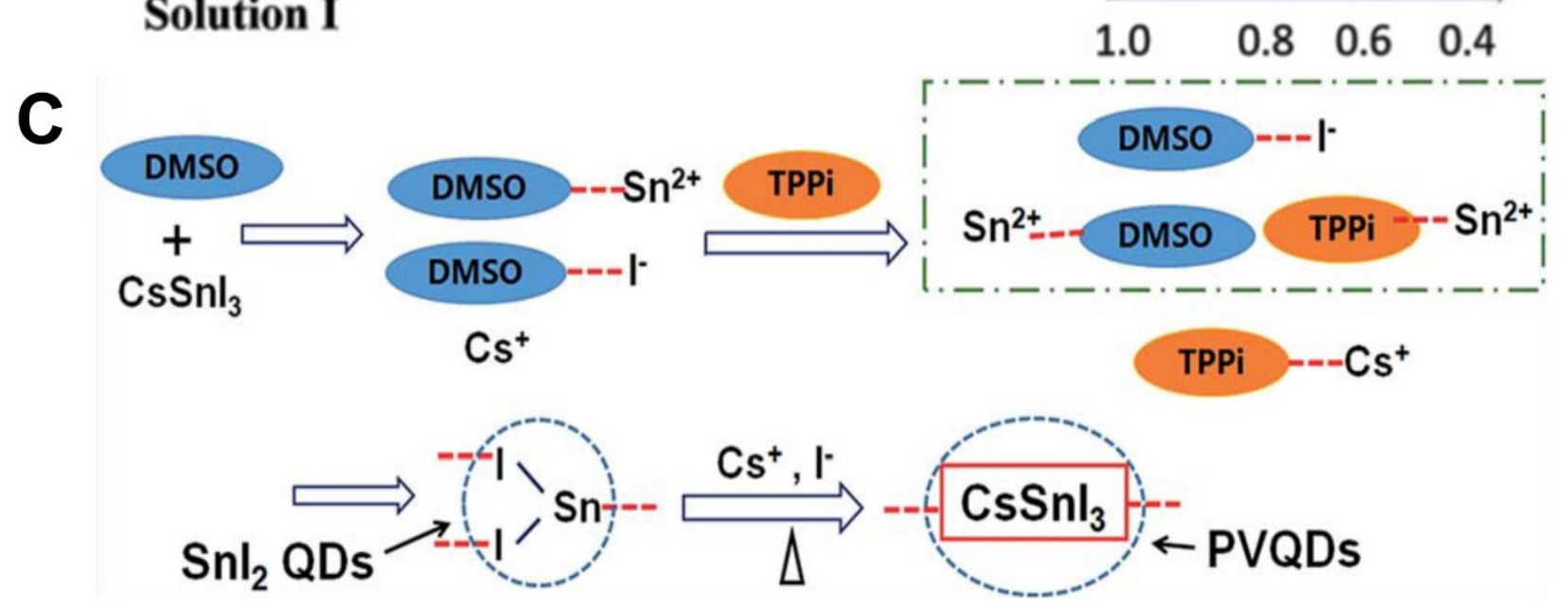

Figure 9. (A) Possible reaction mechanism between hydrazine vapor and Sn-PVSK lattices. Reprinted with permission from Ref. 158. Copyright 2019, American Chemical Society. (B) Preparation process of $\mathrm{MAPb}_{\mathrm{x}} \mathrm{Sn}_{1-\mathrm{x}} \mathrm{I}_{3}$ solution via galvanic displacement reaction between $\mathrm{MAPbI}_{3}$ solution and Sn powders. Reprinted with permission from Ref. 160. Copyright 2019, Wiley-VCH. (C) Schematic procedures of $\mathrm{CsSnI}_{3}$ QDs prepared from TPPi aqueous solution. Reproduced from Ref. 89 with permission from The Royal Society of Chemistry.

Solvent: As the precursor solution of Sn-PVSK is spin-casted onto substrate, the uncontrollable film formation results in two main issues. Firstly, the randomly-oriented small 
crystallites with high densities of GBs severely restrict the charge carriers transport and lower the tolerance for moisture and oxygen as discussed above. Second, the dynamics-dominated process gives rise to inhomogeneous nucleation/growth and discontinuity in thin films, which causes both electrical leakage and device failure. Since the solvent is direct medium of crystallization, the solvent engineering seems the most effective avenue to regulate the film formation by controlling the basic properties such as viscosity, solubility, boiling point, coordination and polarity. The adopted solvent can be classified into good solvents $79,96,142,170-172$ and anti-solvents ${ }^{173-175}$. Generally, the former function to dissolve precursors and regulate the reaction rates among constituents, while the latter are added to lower the solubility during film preparation and aid in homogeneous nucleation and crystal growth. The detailed device performance and stability parameters are summarized in Table 2.

Table 2. Summary of device performance and stability of Sn-PSCs by the solvent strategy

\begin{tabular}{|c|c|c|c|c|c|c|c|}
\hline Active layer & Solvent & $\begin{array}{l}V_{\text {OC }} \\
(\mathrm{V})\end{array}$ & $\begin{array}{c}J_{\mathrm{SC}} \\
\left(\mathrm{mA} / \mathrm{cm}^{2}\right)\end{array}$ & $\begin{array}{l}\text { FF } \\
(\%)\end{array}$ & $\begin{array}{l}\text { PCE } \\
(\%)\end{array}$ & $\begin{array}{c}\text { Stability test } \\
\text { (r.t.) }\end{array}$ & Ref \\
\hline $\mathrm{BA}_{2} \mathrm{MA}_{3} \mathrm{Sn}_{4} \mathrm{I}_{13}$ & $\begin{array}{l}\text { DMSO \& } \\
\text { MAAc }\end{array}$ & 0.38 & 21.9 & 48.3 & 4.0 & $\begin{array}{c}\text { Shelf life, } N_{2}, \\
94 \text { d }(100 \%)\end{array}$ & 79 \\
\hline $\mathrm{MASnI}_{3}$ & $\begin{array}{c}\text { DMSO \& } \\
\text { DMF }\end{array}$ & 0.32 & 21.4 & 46 & 3.2 & N/A & 96 \\
\hline $\mathrm{MAPb}_{0.75} \mathrm{Sn}_{0.25} \mathrm{I}_{3}$ & $\begin{array}{c}\text { DMSO \& } \\
\text { DMF }\end{array}$ & 0.96 & 20.6 & 74 & 14.1 & N/A & 170 \\
\hline $\mathrm{MASn}_{0.25} \mathrm{~Pb}_{0.75} \mathrm{I}_{3}$ & $\begin{array}{c}\text { DMSO \& } \\
\text { DMF }\end{array}$ & 0.81 & 26.4 & 74 & 15.2 & N/A & 171 \\
\hline $\mathrm{FASnI}_{3}$ & $\mathrm{DE}$ & 0.46 & 22.1 & 60.7 & 6.2 & $\begin{array}{c}\text { Shelf life, } \mathrm{N}_{2}, \\
30 \mathrm{~d}(85 \%)\end{array}$ & 45 \\
\hline $\mathrm{FA}_{0.75} \mathrm{MA}_{0.25} \mathrm{SnI}_{3}$ & $\begin{array}{c}\text { Hot CB \& } \\
\text { DMSO vapor }\end{array}$ & 0.55 & 19.4 & 67 & 7.2 & $\begin{array}{c}\text { Shelf life, } \mathrm{N}_{2}, \\
70 \mathrm{~d}(100 \%)\end{array}$ & 172 \\
\hline $\mathrm{FA}_{0.75} \mathrm{MA}_{0.25} \mathrm{SnI}_{3}$ & $\mathrm{CB}$ & 0.55 & 24.3 & 67 & 9.1 & $\begin{array}{l}\text { Shelf life, } \\
\text { encapsulated, } \\
30 \mathrm{~d}(75 \%)\end{array}$ & 173 \\
\hline
\end{tabular}


Note: $\mathrm{MAAc}=$ methylammonium acetate, $\mathrm{DE}=$ diethyl ether, $\mathrm{CB}=$ chlorobenzene.

As for good solvents, the frequently used DMF in Pb-PVSKs is unfavorable in Sn-PVSKs because the greater Lewis acidity of $\mathrm{Sn}^{2+}$ than $\mathrm{Pb}^{2+}$ results in rapid and uncontrollable reaction between $\mathrm{SnX}_{2}$ and AI units. ${ }^{96}$ On the other hand, although DMSO with strong $\mathrm{Sn}^{2+}$ coordination affinity demonstrated effective in modulating the crystallization, its high boiling point and low saturated vapor pressure present a challenge on the complete removal of solvent. Furthermore, recent work by Sargent and coworkers found DMSO and $\mathrm{Sn}^{2+}$ underwent an irreversible redox reaction, thus forming dimethylsulfide and $\mathrm{Sn}^{4+}$ above $100^{\circ} \mathrm{C}$ even under inert atmosphere, which constrains the annealing temperature and makes more difficult to remove the residual DMSO. ${ }^{176}$ The Mathew group reported an initial attempt to replace DMF with DMSO or 2-methoxyethanol as solvent for efficient light harvesting in $\mathrm{CsSnI}_{3}$ based PSCs. ${ }^{142}$ Then the solvent effects on the crystallization was systematically investigated by the Kanatzidis team. ${ }^{96}$ Their results unveiled that the self-assembled $\mathrm{SnI}_{2}$-3DMSO intermediates showed better coordination ability than their $\mathrm{Pb}$ analogues in form of $\mathrm{PbI}_{2}-2 \mathrm{DMSO}$, which explained the origin the uniform, pinhole-free morphology when DMSO were applied as displayed in Figure 10A and B. Impressively, the Choy group revealed the colloidal growth mechanism of precursors in the above mixed solvent, which established the state-of-art protocol of DMF:DMSO $=4: 1$ vol\% in Sn-PVSK optoelectronics. ${ }^{171}$

In comparison to good solvents which serve as a growth rate regulator, anti-solvents such as diethyl ether (DE), toluene (TL), and chlorobenzene (CB) facilitate the homogeneous nucleation. ${ }^{45,172,173}$ It was claimed by Yan et al. that antisolvent dripping of DE is more desirable for synthesizing highly uniform and pinhole-free compact $\mathrm{FASnI}_{3}$ films compared to $\mathrm{CB}$ and TL. ${ }^{45}$ Conversely, Huang and coworkers also compared the morphological effect of these anti-solvents and found that $\mathrm{CB}$ dripping led to better film morphology in $\mathrm{FA}_{0.75} \mathrm{MA}_{0.25} \mathrm{SnI}_{3}$ relative to $\mathrm{DE}$ and 
TL. Despite that the XRD spectra of DE and CB treated samples exhibit similar crystallinity, the CB dripping films show higher PL intensity than those of TL and DE (Figure 10C), indicative of suppressed nonradiative recombination, which is further supported by the slightly longer lifetime extracted from TRPL profiles (Figure 10D). Furthermore, the remarkably larger recombination resistance $\left(\mathrm{R}_{\mathrm{rec}}\right)$ in $\mathrm{CB}$ treated sample confirms the lower trap densities and recombination rates as revealed from the electrochemical impedance spectroscopy (EIS) in Figure 10E and F. The benevolent effect of $\mathrm{CB}$ was interpreted as its high boiling point and the penetration of CB into DMSO-perovskites intermediates during spin-coating. ${ }^{173}$ Such seemingly controversial results may be attributed to either different response to the anti-solvent upon various composition of precursor or the different dripping times of antisolvent. With regards to the work of Yan et al., though no specific dripping time was found, a similar method was used by Park et al. ${ }^{177}$ that DE was dropped slowly in $10 \mathrm{~s}$ before the film became hazy whereas Huang et al. applied CB at the $12^{\text {th }} \mathrm{s}$ of a spin-coating process at $4000 \mathrm{rpm}$ for $60 \mathrm{~s}$. Since $\mathrm{CB}$ possesses both much higher viscosity and boiling point than $\mathrm{DE}, \mathrm{CB}$ tends to reside longer on substrate than $\mathrm{DE}$ during spincoating, which may result in different film formation processes. The shorter residence time of DE also explains why DE necessitates slow and continuous dripping in both Yan and Park's work. 
A

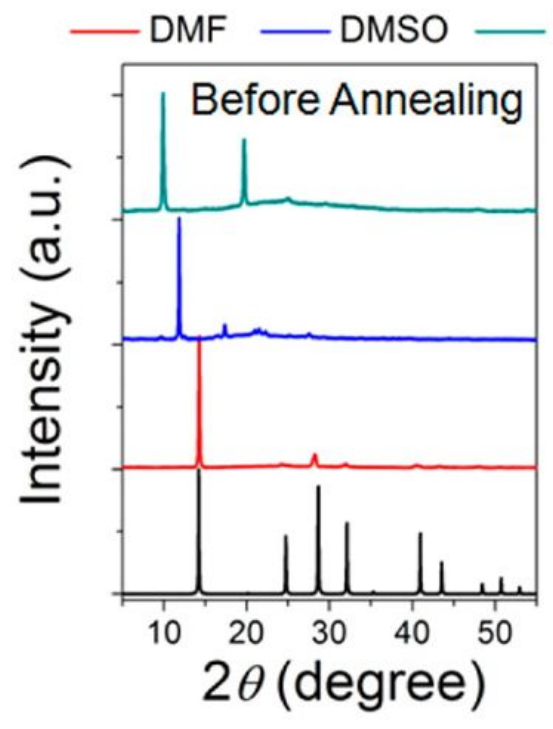

NMP — calc. $\mathrm{CH}_{3} \mathrm{NH}_{3} \mathrm{Snl}_{3}$

B

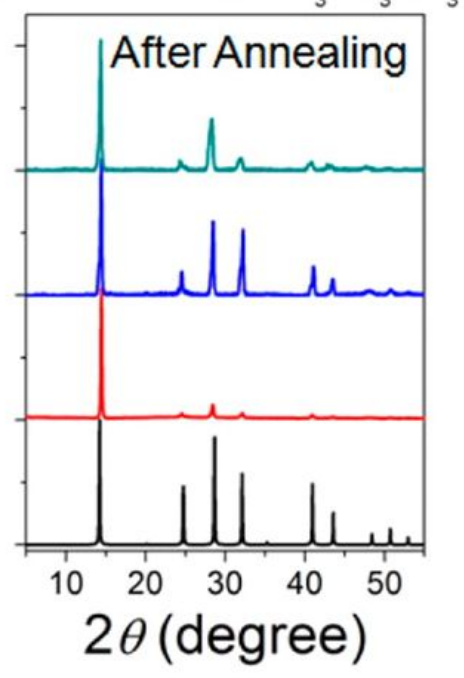

तiforatoge
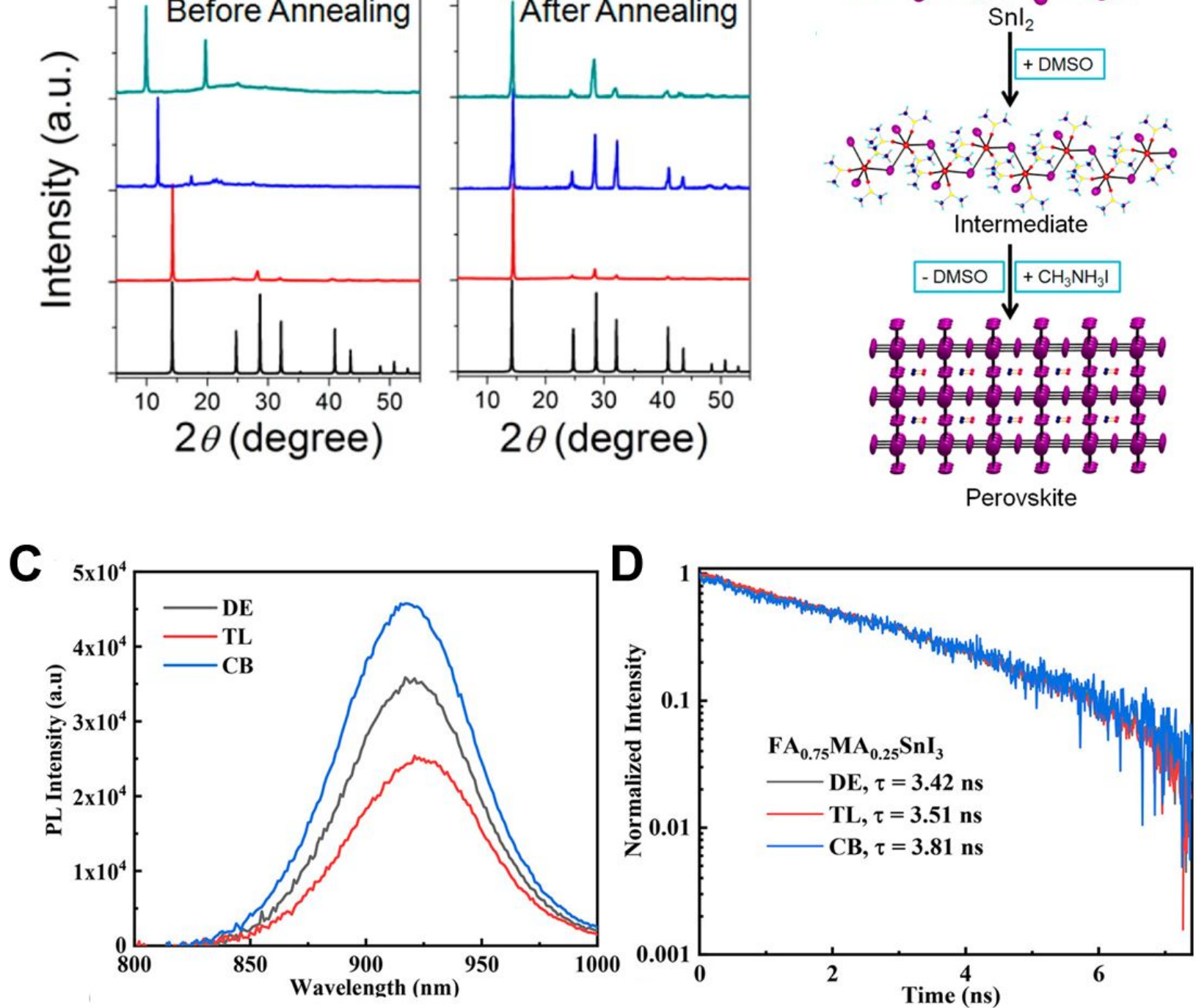

E
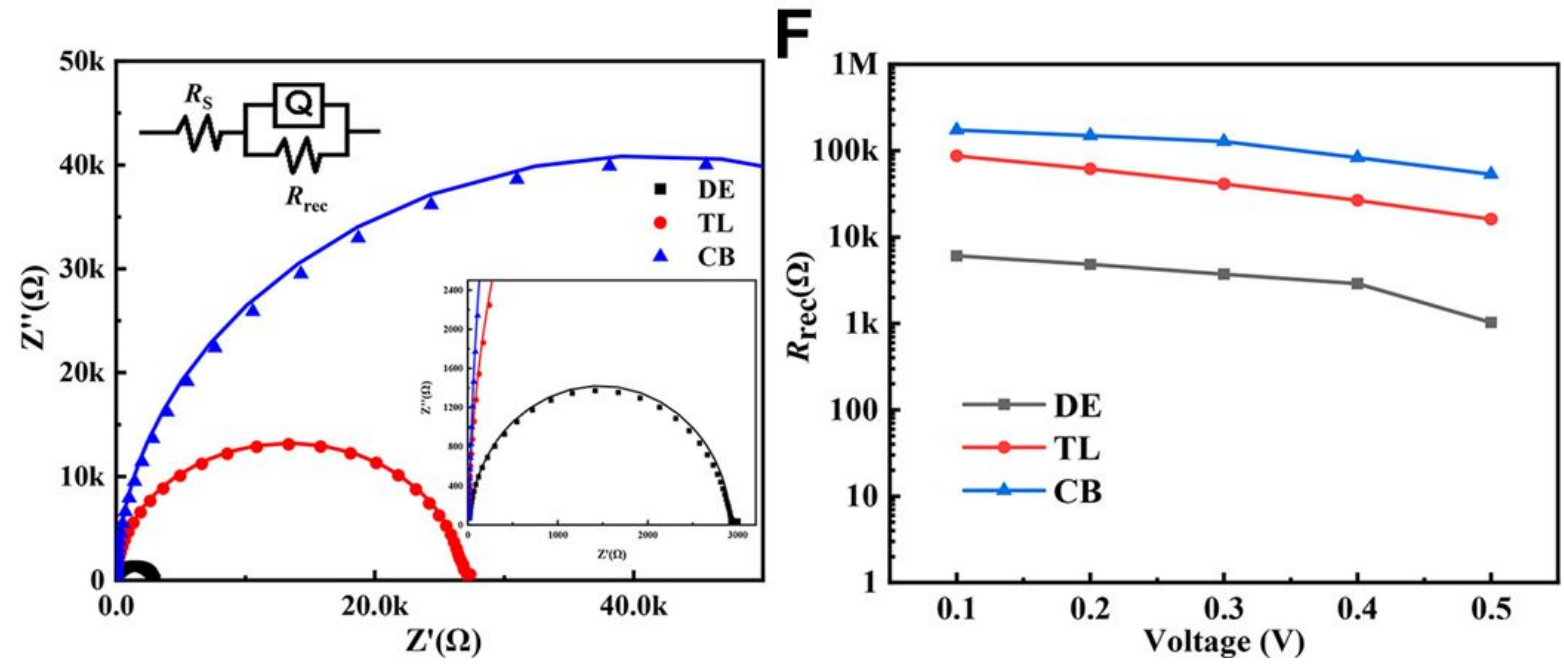
Figure 10. (A) XRD patterns of the $\mathrm{CH}_{3} \mathrm{NH}_{3} \mathrm{SnI}_{3}$ layer with different solvents before (left) and after (right) thermal annealing process at $100^{\circ} \mathrm{C}$ for $15 \mathrm{~min}$. (B) Schematic film formation process starting from $\mathrm{SnI}_{2}$ through the $\mathrm{SnI}_{2}$-3DMSO intermediate. (A, B) Reprinted with permission from Ref. 96. Copyright 2019, American Chemical Society. (C) Steady-state PL spectra and (D) TRPL spectra of the encapsulated $\mathrm{FA}_{0.75} \mathrm{MA}_{0.25} \mathrm{SnI}_{3}$ films with different antisolvents on quartz substrates. (E) Nyquist plots of $\mathrm{FA}_{0.75} \mathrm{MA}_{0.25} \mathrm{SnI}_{3}$-based PSCs with different antisolvents dripping measured at $0.4 \mathrm{~V}$ under dark conditions. Inset: Zoomed-in patterns and the equivalent circuit model for fitting the plots. (F) Fitted $\mathrm{R}_{\mathrm{rec}}$ at different applied voltages obtained from the EIS analysis. (C-F) Reprinted with permission from Ref. 173. Copyright 2019, American Chemical Society.

Preparation Methodology: In addition to the additive and solvent engineering, alternate methodologies have been developed to enrich the pathways of crystallization control and oxidation inhabitation for high-quality Sn-PVSK thin films. The conventional one-step spin-coating method fails to separate the nucleation and crystal growth processes, which lead to both random crystal orientation and poor film morphology in Sn-PVSKs if external intervention is not applied. In contrast, multi-step sequential deposition by either spin-coating, ${ }^{174}$ thermal evaporation ${ }^{178-181}$ or post-ion exchange ${ }^{40}$ artificially fixes the spatial location of reaction sites and manipulates the reaction steps among precursors. For instance, the Kanatzidis group proposed a multichannel interdiffusion protocol by spin-coating of FAI/PEDOT:PSS polymer layer followed by thermal evaporation of $\mathrm{SnI}_{2}$ to improve both the film coverage and crystallinity as shown in Figure $11 \mathrm{~A} .{ }^{178}$ Most recently, Jiang et al. presented a novel two-step cation exchange method (Figure 11B) where reducing hydrazinium $\left(\mathrm{HA}^{+}\right)$based PVSKs was firstly deposited to prevent the oxidation of $\mathrm{Sn}^{2+} .40$ Then, the film underwent cation exchange from $\mathrm{HA}^{+}$to $\mathrm{MA}^{+}$to generate highly uniform $\mathrm{MASnI}_{3}$ thin films. The resulting devices delivered an excellent PCE of 7.13\%. In compared to spin-coating, the vapor-phase sequential deposition features better stoichiometric accuracy and higher crystallinity. Fan and coworkers applied chemical vapor deposition to fabricate $\mathrm{Sn}-\mathrm{Pb}$ alloyed PVSKs with large grain sizes up to $5 \mu \mathrm{m} .{ }^{180}$ In another work, the Lunt group realized all thermal vapor-deposited $\mathrm{CsSnBr}_{3}$ PSCs, which showed an outstanding stability against air and under light 
illumination. ${ }^{179}$ The improved durability of device prepared by a two-step vapor deposition method may arise from both decreased defects and GBs (enlarged crystals) owing to the thermodynamic dominated reaction among precursors. Most recently, Tao and coworkers employed a novel recrystallization process to prepare $\mathrm{FASnI}_{3}$ single crystals, which showed an outstanding airstability resulting from suppressed GBs. Such a strategy is helpful to improve both long-term storage stability and reproducibility. ${ }^{182}$
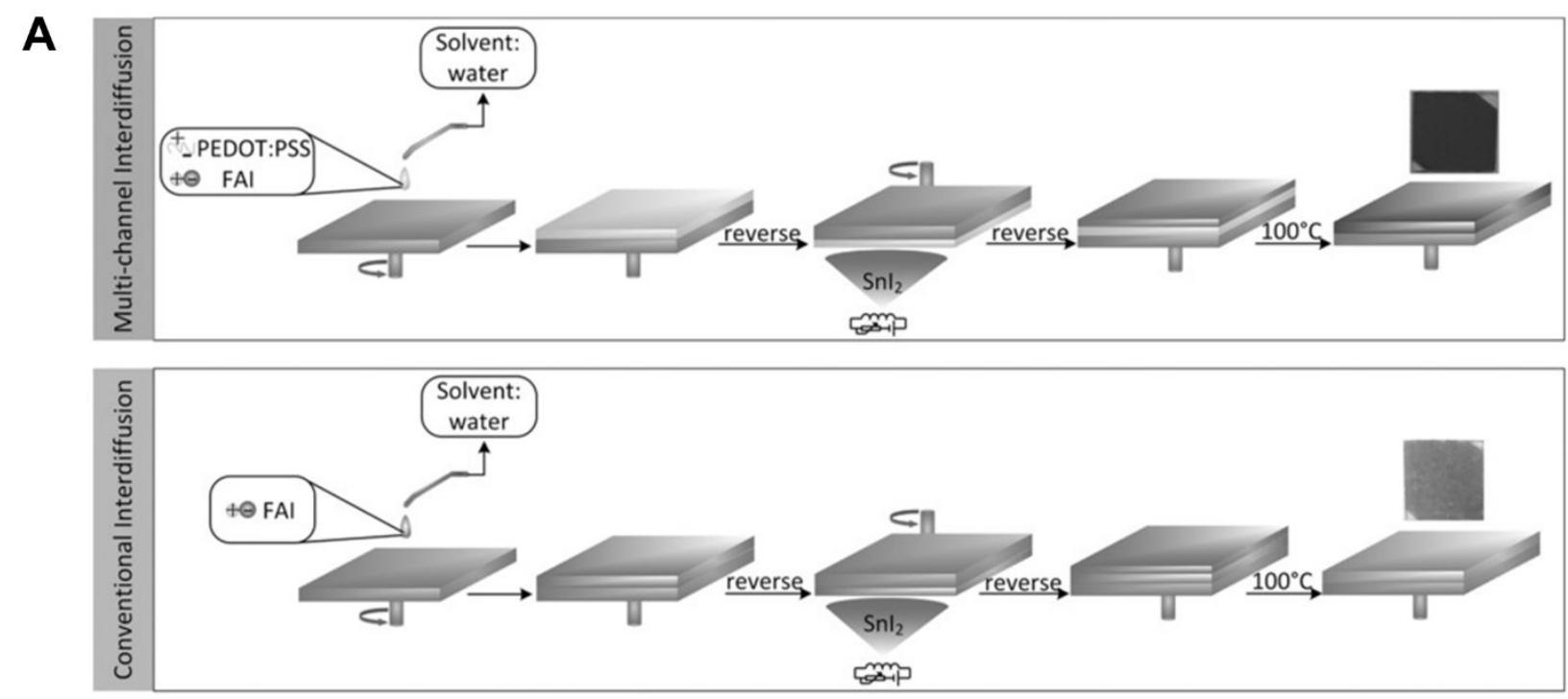

B

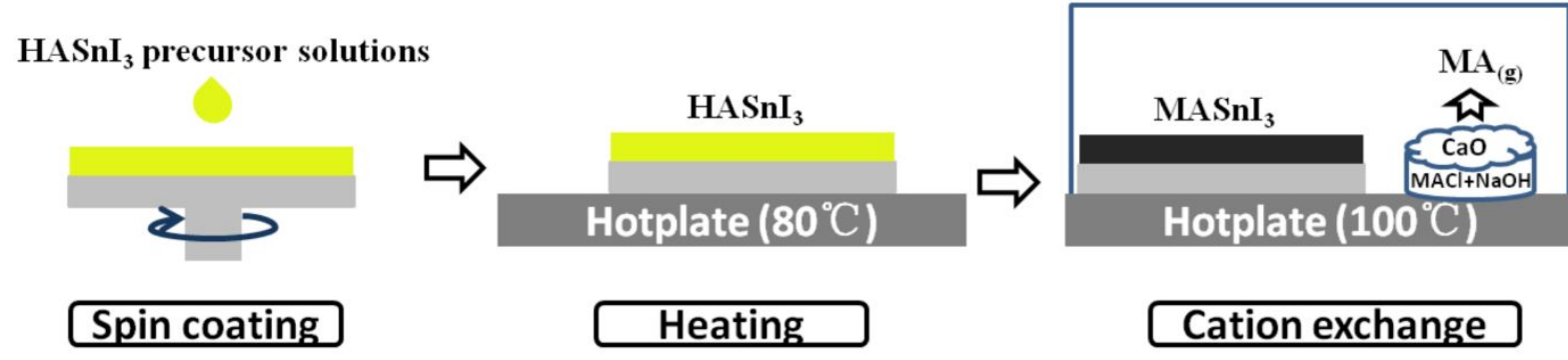

Figure 11. Schematic illustration of (A) multichannel interdiffusion process $\left(60 \mathrm{mg} \mathrm{mL}^{-1}\right.$ FAI dissolved in 20\% PEDOT:PSS aqueous solution. Reprinted with permission from Ref. 178. Copyright 2019, Wiley-VCH. Copyright 2019, Wiley-VCH. (B) Cation exchange process from $\mathrm{MA}^{+}$to $\mathrm{HA}^{+}$. Reprinted with permission from Ref. 40. Copyright 2019, Wiley-VCH.

Compositional Modulation: As aforementioned, the A/B/X site cations significantly affect the electronic structure, thermal and phase stability of a-prepared Sn-PVSKs. In this section, we will 
discuss the compositional engineering on $\mathrm{A}, \mathrm{B}$ and $\mathrm{X}$ site, respectively. The detailed device performance and stability parameters are summarized in Table 3.

Table 3. Summary of device performance and stability of Sn-PSCs by the composition strategy

\begin{tabular}{|c|c|c|c|c|c|c|c|}
\hline Active layer & Strategy & $\begin{array}{l}V_{\mathrm{OC}} \\
(\mathrm{V})\end{array}$ & $\begin{array}{c}J_{\mathrm{SC}} \\
\left(\mathrm{mA} / \mathrm{cm}^{2}\right)\end{array}$ & $\begin{array}{l}\text { FF } \\
(\%)\end{array}$ & $\begin{array}{c}\text { PCE } \\
(\%)\end{array}$ & $\begin{array}{l}\text { Stability } \\
\text { test (r.t.) }\end{array}$ & Ref. \\
\hline $\mathrm{HA}_{0.2} \mathrm{MA}_{0.8} \mathrm{SnI}_{3}$ & & 0.35 & 11.8 & 50 & 2.6 & $\begin{array}{c}\text { Shelf life, } \\
\mathrm{N}_{2}, 5 \mathrm{~d} \\
(90 \%)\end{array}$ & 68 \\
\hline $\mathrm{Cs}_{0.08} \mathrm{FA}_{0.92} \mathrm{SnI}_{3}$ & $\begin{array}{l}\text { Mixed } \\
\text { A-Site }\end{array}$ & 0.44 & 20.7 & 67 & 6.1 & $\begin{array}{l}\text { Shelf life: } \\
\mathrm{N}_{2}, 2000 \mathrm{~h} \\
(90 \%)\end{array}$ & 186 \\
\hline $\mathrm{FA}_{0.75} \mathrm{MA}_{0.25} \mathrm{SnI}_{3}$ & & 0.61 & 21.2 & 63 & 8.1 & $\begin{array}{l}\text { Shelf life, } \\
\mathrm{N}_{2}, 400 \mathrm{~h} \\
(80 \%)\end{array}$ & 190 \\
\hline $\mathrm{CsSn}_{0.5} \mathrm{Ge}_{0.5} \mathrm{I}_{3}$ & & 0.63 & 18.6 & 61 & 7.1 & $\begin{array}{l}\text { Continuous } \\
\text { operation, } \\
\mathrm{N}_{2}\left(45^{\circ} \mathrm{C}\right), \\
500 \mathrm{~h}(92 \%)\end{array}$ & 64 \\
\hline $\mathrm{MASn}_{0.15} \mathrm{~Pb}_{0.85} \mathrm{I}_{3}$ & & 1.02 & 20.8 & 59 & 12.5 & $\mathrm{~N} / \mathrm{A}$ & 159 \\
\hline $\mathrm{MAPb}_{0.38} \mathrm{Sn}_{0.62} \mathrm{I}_{3}$ & $\begin{array}{l}\text { Mixed } \\
\text { B-Site }\end{array}$ & 0.80 & 25.5 & 69 & 14.0 & $\begin{array}{l}\text { Continuous } \\
\text { operation, } \\
\text { encapsulate } \\
\text { d, } 100 \mathrm{~h} \\
(90 \%)\end{array}$ & 180 \\
\hline $\mathrm{MASn}_{0.5} \mathrm{~Pb}_{0.5} \mathrm{I}_{3}$ & & 0.58 & 20.6 & 60 & 7.3 & $\mathrm{~N} / \mathrm{A}$ & 97 \\
\hline $\mathrm{MASn}_{0.5} \mathrm{~Pb}_{0.5} \mathrm{I}_{3}$ & & 0.42 & 20.0 & 50 & 4.2 & $\mathrm{~N} / \mathrm{A}$ & 191 \\
\hline $\mathrm{FASn}_{0.5} \mathrm{~Pb}_{0.5} \mathrm{I}_{3}$ & & 0.70 & 28.4 & 55 & 10.8 & $\begin{array}{c}\text { Shelf life, } \\
\mathrm{N}_{2}, 100 \mathrm{~h} \\
(85 \%)\end{array}$ & 195 \\
\hline $\mathrm{MASnIBr}_{2}$ & $\begin{array}{l}\text { Mixed } \\
\text { X-Site }\end{array}$ & 0.82 & 12.3 & 57 & 5.7 & $\begin{array}{l}\text { Continuous } \\
\text { operation, } \\
\text { encapsulate } \\
\text { d, } 12 \mathrm{~h} \\
(80 \%)\end{array}$ & 16 \\
\hline $\mathrm{CsSnI}_{2} \mathrm{Br}$ & & 0.29 & 15.1 & 38 & 1.7 & $\mathrm{~N} / \mathrm{A}$ & 201 \\
\hline \multicolumn{8}{|c|}{41} \\
\hline & & rag & Plus Enviror & & & & \\
\hline
\end{tabular}




\begin{tabular}{|c|c|c|c|c|c|c|c|}
\hline $\mathrm{MASnIBr}_{1.8} \mathrm{Cl}_{0.2}$ & & 0.41 & 14.0 & 67 & 5.5 & $\begin{array}{c}\text { Shelf life, } \\
\text { encapsulate } \\
\text { d, maintain } \\
2000 \mathrm{~h} \\
(90 \%) \\
\text { Continuous } \\
\text { operation, } \\
\text { encapsulate } \\
\text { d, } 1000 \mathrm{~h} \\
(83 \%)\end{array}$ & 202 \\
\hline $\begin{array}{l}\mathrm{FA}_{0.75} \mathrm{MA}_{0.25} \\
\mathrm{Sn}_{0.95} \mathrm{Ge}_{0.05} \mathrm{I}_{3}\end{array}$ & \multirow{5}{*}{$\begin{array}{c}\text { Mixed } \\
\text { A/B-Site }\end{array}$} & 0.45 & 21.9 & 70 & 6.0 & $\begin{array}{l}\text { Shelf life, } \\
\text { Air, } 60 \text { min } \\
\quad(80 \%)\end{array}$ & 52 \\
\hline $\mathrm{MA}_{0.4} \mathrm{FA}_{0.6} \mathrm{~Pb}_{0.4} \mathrm{Sn}_{0.6} \mathrm{I}_{3}$ & & 0.80 & 26.9 & 71 & 15.1 & N/A & 187 \\
\hline $\mathrm{FA}_{0.7} \mathrm{Cs}_{0.3} \mathrm{~Pb}_{0.7} \mathrm{Sn}_{0.3} \mathrm{I}_{3}$ & & 0.77 & 26.4 & 72 & 14.6 & N/A & 188 \\
\hline $\mathrm{MA}_{0.4} \mathrm{FA}_{0.6} \mathrm{Sn}_{0.6} \mathrm{~Pb}_{0.4} \mathrm{I}_{3}$ & & 0.83 & 27.3 & 80 & 17.8 & $\mathrm{~N} / \mathrm{A}$ & 189 \\
\hline $\mathrm{MA}_{0.4} \mathrm{FA}_{0.6} \mathrm{~Pb}_{0.4} \mathrm{Sn}_{0.6} \mathrm{I}_{3}$ & & 0.85 & 28.5 & 73 & 17.6 & $\begin{array}{c}\text { Shelf life, } \\
\text { encapsulate } \\
\text { d, 33d } \\
(94 \%)\end{array}$ & 198 \\
\hline $\begin{array}{l}\mathrm{MAPb}_{0.9} \mathrm{Sn}_{0.05} \\
\mathrm{Cu}_{0.05} \mathrm{I}_{2.9} \mathrm{Br}_{0.1}\end{array}$ & & 1.09 & 23.9 & 81 & 21.1 & $\begin{array}{c}\text { Shelf life, } \\
\mathrm{N}_{2}, 70 \mathrm{~h} \\
(60 \%)\end{array}$ & 13 \\
\hline $\begin{array}{c}\mathrm{MAPb}_{0.5} \mathrm{Sn}_{0.5} \\
\mathrm{I}_{2.4} \mathrm{Br}_{0.6}\end{array}$ & & 0.90 & 25.9 & 76 & 17.6 & $\begin{array}{c}\text { Shelf life, } \\
30-50 \% \\
\text { RH, 14d } \\
(44 \%)\end{array}$ & 193 \\
\hline $\mathrm{CsPb}_{0.9} \mathrm{Sn}_{0.1} \mathrm{IBr}_{2}$ & $\begin{array}{c}\text { Mixed } \\
\text { B/X-Site }\end{array}$ & 1.26 & 14.3 & 63 & 11.3 & $\begin{array}{l}\text { Shelf life, } \\
\text { encapsulate } \\
\text { d, } 3 \text { months } \\
(100 \%)\end{array}$ & 194 \\
\hline $\mathrm{MAPb}_{0.75} \mathrm{Sn}_{0.25} \mathrm{I}_{1.2} \mathrm{Br}_{1.8}$ & & 1.04 & 15.5 & 78 & 12.6 & $\begin{array}{c}\text { Shelf life, } \\
\mathrm{N}_{2}, 30 \mathrm{~d} \\
(95 \%)\end{array}$ & 196 \\
\hline $\mathrm{MAPb}_{0.85} \mathrm{Sn}_{0.15} \mathrm{I}_{1.3} \mathrm{Cl}_{1.7}$ & & 0.76 & 19.1 & 66 & 10.1 & $\mathrm{~N} / \mathrm{A}$ & 197 \\
\hline $\begin{array}{c}\mathrm{MA}_{0.15} \mathrm{FA}_{0.85} \mathrm{~Pb}_{0.6} \mathrm{Sn}_{0.4} \\
\mathrm{I}_{2.55} \mathrm{Br}_{0.45}\end{array}$ & $\begin{array}{l}\text { Mixed } \\
\text { A/B/X- }\end{array}$ & 0.87 & 26.4 & 79 & 18.2 & $\begin{array}{l}\text { Shelf life, } \\
50 \% \mathrm{RH},\end{array}$ & 160 \\
\hline
\end{tabular}


A-Site: In comparison to B-site cation and $\mathrm{X}$-site halide anion, which jointly determine the electronic structure, the A-site cation seems to impact less on the electronic structure of Sn$\mathrm{PVSKs}^{95}$ although A-site cations may affect $E_{\mathrm{g}}$ to some extent because of their contribution to the density of states ${ }^{106}$ or spin-orbit coupling via distorting octahedra. ${ }^{58}$ Importantly, the ambient structural stabilities of PVSKs were found to strongly correlated with the A-site cations. $^{41,42,58-60,183}$ As mentioned in Section 2, the incorporation of A-site cation with different ion radius results in variations of tolerance factor and remarkable changes in both crystal symmetry and phase stability of $\mathrm{ABX}_{3}$. Moreover, the practical applications in Sn-PSCs put forward various requirements for the intrinsic properties such as $E_{\mathrm{g}}$ and thermal stability, which cannot be fulfilled with single A-site cation. Therefore, the mixed A-site cation strategy emerges an effective method to combine the advantages with good miscibility of multicomponent precursors. ${ }^{21,184-190}$ For instance, Cs doping is adopted in $\mathrm{FASnI}_{3}$ system to adjust the tolerance factor back to unity and improve phase stability along with structural symmetry. ${ }^{186}$ Such doping process is energetically favorable since theoretical results showed decreased free energy upon Cs introduction. Moreover, McGehee at al. identified that the incorporation of $\mathrm{Cs}^{+}$will not change the cubic symmetry of $\mathrm{FASnI}_{3}$ and investigated the influences of two competing A/B-site cations related mechanisms — octahedral tilting and lattice contraction—on the $E_{\mathrm{g}}$ of PVSKs as shown in Figure $12 \mathrm{~A}-\mathrm{C}$, which widens and narrows the $E_{\mathrm{g}}$, respectively, owing to the different orbital overlaps between Sn-5s and I-5p. ${ }^{189}$ Besides Cs incorporation, $\mathrm{FA}^{+}$was also found to increase the thermal stability $^{190}$ and oxidation resistance ${ }^{43}$ in MA-based Sn-PVSKs. Interestingly, the hydrazinium (HA), which was outlined above as either antioxidants ${ }^{165}$ or intermediate components ${ }^{40}$ in $\mathrm{Sn}$ PVSK thin films, was also explored to partially substitute MA for better photo-stability in inert 
atmosphere when compared to the reference $\mathrm{MASnI}_{3}$ films. ${ }^{68}$ To sum, the mixed cation strategy is a viable avenue to encompass all the merits of single A-site cation at one strike. Various functional groups such as reducing double bonds or coordinating endings may be worth to incorporate into their molecule structures to tailor the optoelectronic properties and stability of $\mathrm{ASnX}_{3}$ provided that the tolerance factor is satisfied.

B-Site: One of the fundamental aspects towards higher performance of PSCs is to broaden the absorption range of the active layer to the near-infrared region. Since the conduction band minimum $(\mathrm{CBM})$ and the valence band maximum $(\mathrm{VBM})$ are determined by the anti-bonding states of B-p/X-s and B-s/X-p electrons, respectively, it is feasible to engineer the optical $E_{\mathrm{g}}$ by adjusting the B-site cation such as alloying $\mathrm{Pb}$ into Sn-PVSKS to make the full use of solar light absorption. Despite that the Sn-PVSKs exhibit significantly narrower $E_{\mathrm{g}} \mathrm{s}$ than $\mathrm{Pb}$ analogues, it was found that that the $E_{\mathrm{g}} \mathrm{s}$ of $\mathrm{Pb} / \mathrm{Sn}$ alloys deviated from the Vegard's law with a minimum falling between the neat $\mathrm{Sn}$ and $\mathrm{Pb}$ phases as discussed in the previous section, which paves up a smooth way to the feasibility of high-Sn content or even pure Sn-PSCs. In this part, we seek to summarize recent advances in the $\mathrm{Pb} / \mathrm{Sn}$ alloyed PVSKs, ${ }^{146,191-198}$ and then present the emerging $\mathrm{Sn} / \mathrm{Ge}$ binary systems. ${ }^{64,117,199,}$

The Hayase group firstly reported the preparation of $\mathrm{MASn}_{0.5} \mathrm{~Pb}_{0.5} \mathrm{I}_{3} \mathrm{PVSK}$ with an extended absorption edge up to $1060 \mathrm{~nm}$, which red-shifted $260 \mathrm{~nm}$ than benchmark $\mathrm{MAPbI}_{3}$, verifying the beneficial influence of Sn addition on $E_{\mathrm{g}} \mathrm{s} .{ }^{191}$ Later, the Kanatzidis team unveiled the abnormal $E_{\mathrm{g}}$ evolution of alloyed $\mathrm{Pb} / \mathrm{Sn} \mathrm{PVSKs}$, which was presumably attributed to a systematic change in the atomic orbital composition of the conduction and valence bands. ${ }^{97}$ For detailed discussions on such anomalous $E_{\mathrm{g}}$ evolution trend, please refer to Section 3. Although the origin of the nonlinearity is still under debate, the experimental method to realize B-site alloying has been demonstrated by 
both the Ginger ${ }^{146}$ and Fan ${ }^{180}$ via low-temperature ion exchange and chemical vapor deposition, respectively, which opens up a new means towards iso-valent and hetero-valent ion substitution in Sn-PVSKs. Since then, the device performance of Sn-PSCs began to surge by-tailoring the B-site composition. ${ }^{192-197}$ For instance, the Jen group gradually increased the $\mathrm{Sn}$ content in the $\mathrm{Pb} / \mathrm{Sn}$ binary PVSKs and boosted the PCE from $10.1 \%$ (15 mol\% Sn), ${ }^{197} 12.59 \%(25 \mathrm{~mol} \% \mathrm{Sn}){ }^{196}$ up to $17.63 \%$ (50 mol\% Sn). ${ }^{192}$ The Yan group further increased the Sn ratio to $60 \mathrm{~mol} \%$ and utilized the formed $\mathrm{Pb} / \mathrm{Sn}$ alloyed PVSK as the bottom cell in all-PVSK tandem solar cells. ${ }^{198}$ Consequently, the stacked 4-terminal-all-perovskite tandem solar cells show a steady-state efficiency of $21.0 \%$. 


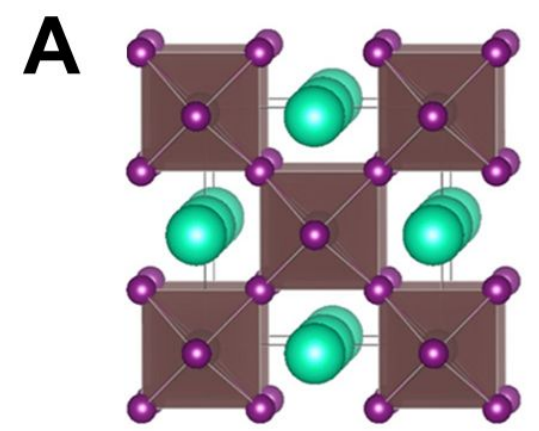

Lattice Contraction

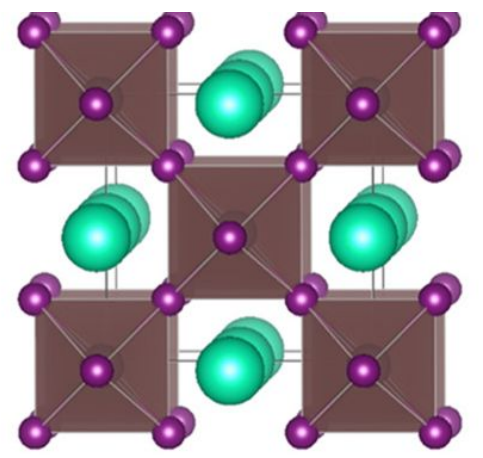

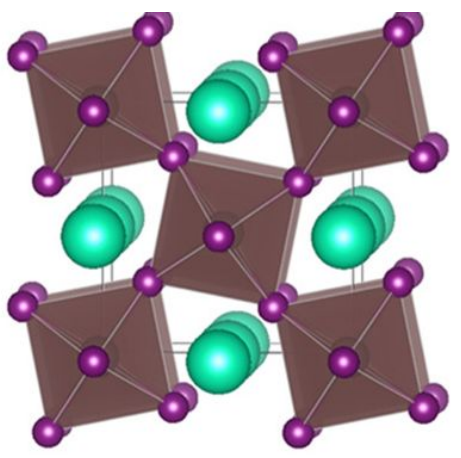

Octahedral Tilting 
further increased up to $6.90 \%$ after aging for $72 \mathrm{~h}$, which outperformed the pure Sn-based reference device (3.31\%). Afterwards, thermally stimulated current (TSC) was exploited by Hayase et al. to unravel that Ge addition efficiently reduced the trap states from $10^{15}-10^{17} \mathrm{~cm}^{-3}$ to $10^{8}-10^{14} \mathrm{~cm}^{-3}$, thereby affording longer charge diffusion length $(\sim 1 \mu \mathrm{m})$ and lifetime $(5.04 \mathrm{~ns})$ coupled with excellent charge mobility $\left(98.27 \mathrm{~cm}^{2} \mathrm{~V}^{-1} \mathrm{~s}^{-1}\right) \cdot{ }^{117}$ Besides $E_{\mathrm{g}}$ tuning and defects passivation, Padture and coworkers further demonstrated the in-situ encapsulation effect of Sn/Ge binary system. ${ }^{64}$ It was found the $\mathrm{Ge}^{2+}$ may drift to the surface during operation and spontaneously generated nativeoxide, i.e., Sn-doped $\mathrm{GeO}_{2}$ layer, which protected the devices from oxygen incursion as indicated from the depth-dependent XPS and the ambient aging profile in Figure 13A and B. Consequently, the optimal $\mathrm{CsSn}_{0.5} \mathrm{Ge}_{0.5} \mathrm{I}_{3}$ film showed longer ambient stability than reference $\mathrm{CsSnI}_{3}$ or even $\mathrm{Pb}$ analogues (Figure 13C) and the resulting PSCs exhibited a promising PCE of 7.11\% and outstanding stability which maintained $92 \%$ of the initial PCE after continuous operation in $\mathrm{N}_{2}$ for $500 \mathrm{~h}$.

$X$-Site: Since the $\mathrm{np}^{2}$ electrons of halide anions contribute to the electronic structure of SnPVSKs, the $E_{\mathrm{g}}$ tuning may be realized by mixed X-site strategies. For instance, it was reported the $E_{\mathrm{g}}$ of $\mathrm{MASnX}_{3}$ can be tailored over a large range of the visible spectrum with different $\mathrm{Br}^{-} / \mathrm{Cl}^{-} / \mathrm{I}^{-}$ ratio. ${ }^{200}$ Figure 13D and E show the energy diagrams and absorption spectra of MA based SnPVSKs depending on various halide combinations. Similar phenomenon was also observed $\mathrm{Br}^{-}$ doped $\mathrm{CsSnI}_{3}$. However, structural evolution from orthorhombic to cubic lattices was observed as the $\mathrm{Br}^{-}$content increased, ${ }^{201}$ which may cause unexpected results during operation. Additionally, the octahedral factor is directly related to X-site halogen ions and hence a rational combination of different X-site halogen anion might significantly improve phase stability of Sn-PVSKs. The effect of mixing halides on the phase stability was investigated Diau team. ${ }^{200}$ The results showed that 
large radius difference between $\mathrm{Cl}^{-}$and $\mathrm{I}^{-}$induced incomplete solid solutions in $\mathrm{MASnI}_{3-\mathrm{x}} \mathrm{Cl}_{\mathrm{x}}$, which could be mediated by incorporation of middle-sized $\mathrm{Br}^{-}$to form stable tri-halide Sn-PVSKs.
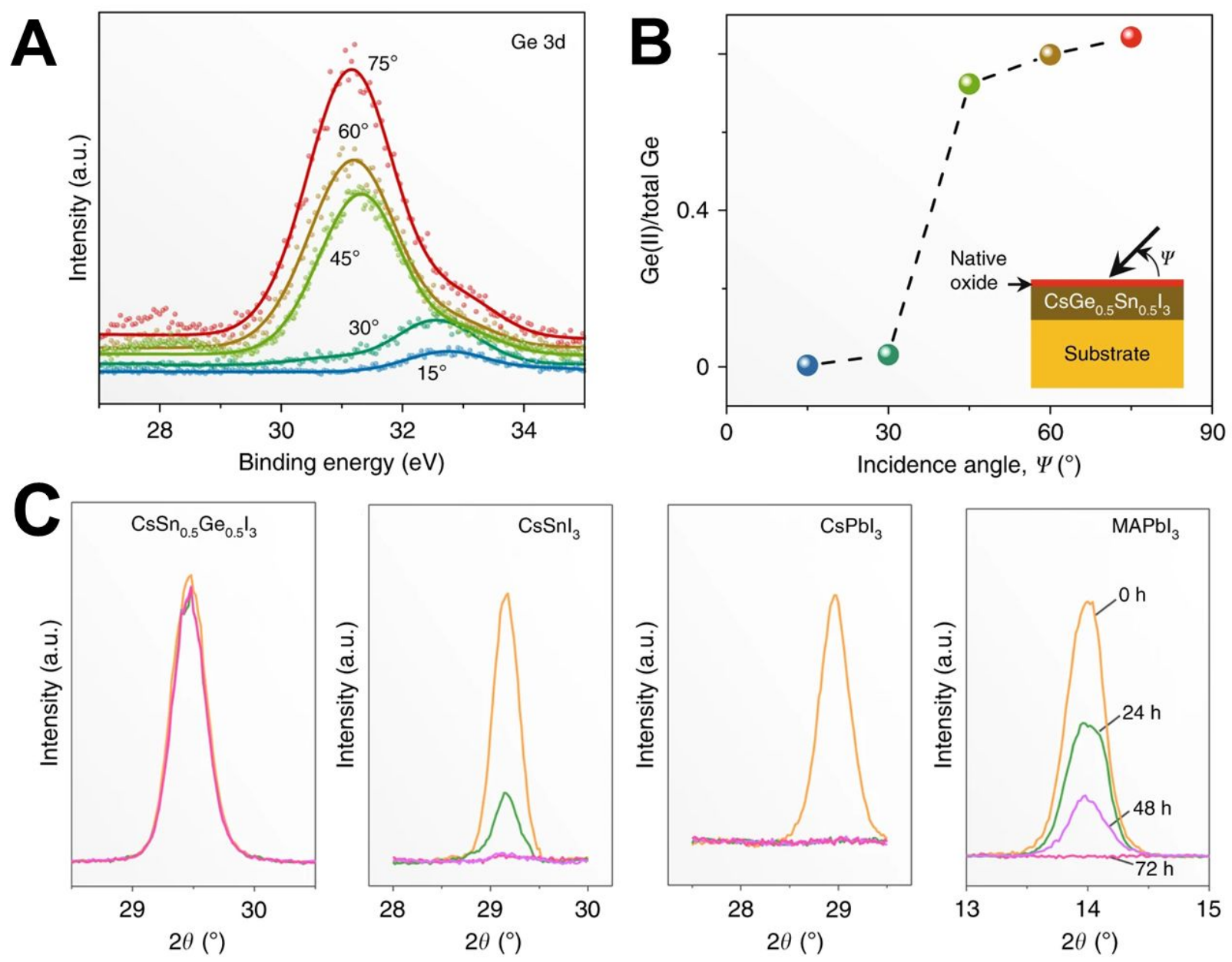

D
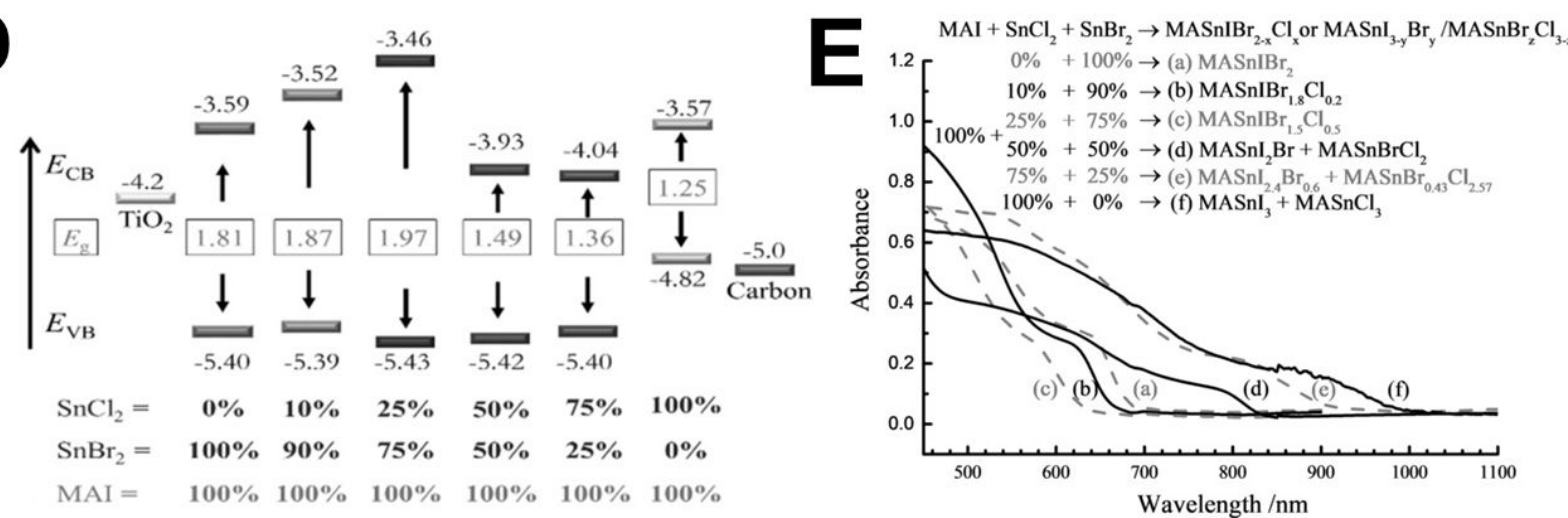

Figure 13. (A) XPS characterization of Ge $3 \mathrm{~d}$ XPS spectra of $\mathrm{CsSn}_{0.5} \mathrm{Ge}_{0.5} \mathrm{I}_{3}$ PVSK thin film at different incidence angles and (B) corresponding plot of the $\mathrm{Ge}^{2+}$ content vs. the incidence angle. (C) XRD patterns of PVSK thin films before and after exposure for 24, 48, and $72 \mathrm{~h}$ to lightsoaking (1 sun) at approximately $45{ }^{\circ} \mathrm{C}$ and $80 \% \mathrm{RH}$. From left to right: $\mathrm{CsSn}_{0.5} \mathrm{Ge}_{0.5} \mathrm{I}_{3}, \mathrm{CsSnI}_{3}$, $\mathrm{CsPb}_{3}$, and $\mathrm{MAPbI}_{3}$. (D) Energy diagrams of mixed-halide Sn-PVSK films with various MAI and 
$\mathrm{SnCl}_{2} / \mathrm{SnBr}_{2}$ ratios. (E) The absorption spectra of mixed halide $\mathrm{Sn}$ based PVSKs on glass substrates. (D, E) Reprinted with permission from Ref. 200. Copyright 2019, Wiley-VCH.

Interfacial Band Alignment: The afore-mentioned strategies focused on preparing ambient stable and high-quality Sn-PVSK. However, the larger variation of VBM and CBM in Sn-PVSK from those of $\mathrm{Pb}$ will cause band alignment mismatch between the charge-extraction and the active layers, ${ }^{64}$ which results in severe $V_{\mathrm{OC}}$ loss in photovoltaic devices. Therefore, the optimization of band alignment is crucial to enhance device performance. In this section, we will summarize recent advances in this field in order to compare the pros/cons of different HTLs and ETLs. The detailed device performance and stability parameters are summarized in Table 4.

Table 4. Summary of device performance and stability of Sn-PSCs by the interlayer strategy

\begin{tabular}{|c|c|c|c|c|c|c|c|}
\hline Active Layer & $\begin{array}{c}\text { Interfacial } \\
\text { layer }\end{array}$ & $\begin{array}{l}V_{\text {OC }} \\
(V)\end{array}$ & $\begin{array}{c}J_{\mathrm{SC}} \\
\left(\mathrm{mA} / \mathbf{c m}^{2}\right)\end{array}$ & $\begin{array}{l}\text { FF } \\
(\%)\end{array}$ & $\begin{array}{l}\text { PCE } \\
(\%)\end{array}$ & $\begin{array}{l}\text { Stability } \\
\text { Test (r.t.) }\end{array}$ & Ref. \\
\hline $\mathrm{FAPb}_{0.75} \mathrm{Sn}_{0.25} \mathrm{I}_{3}$ & $\mathrm{NiO}_{\mathrm{x}}$ & 0.81 & 28.2 & 75 & 17.3 & $\begin{array}{c}\text { Shelf life, } N_{2}, \\
46 \text { d }(92 \%)\end{array}$ & 204 \\
\hline $\mathrm{FASn}_{0.5} \mathrm{~Pb}_{0.5} \mathrm{I}_{3}$ & PCP-Na & 0.78 & 28.5 & 73 & 16.3 & $\mathrm{~N} / \mathrm{A}$ & 205 \\
\hline $\mathrm{FASnI}_{3}$ & $\begin{array}{c}\text { PEG- } \\
\text { PEDOT:PSS }\end{array}$ & 0.37 & 22.1 & 63 & 5.1 & $\begin{array}{c}\text { Shelf life, } N_{2}, \\
30 \text { d }(95 \%)\end{array}$ & 208 \\
\hline $\mathrm{FA}_{0.6} \mathrm{Sn}_{0.6} \mathrm{~Pb}_{0.4} \mathrm{I}_{3}$ & $\begin{array}{c}\text { PBDB- } \\
\text { T:ITIC/ } \\
\text { PEDOT:PSS }\end{array}$ & 0.86 & 27.9 & 75 & 18.0 & $\begin{array}{c}\text { Shelf life, } N_{2}, \\
600 \text { h }(80 \%)\end{array}$ & 209 \\
\hline $\mathrm{FASnI}_{3}$ & LDP & 0.45 & 24.9 & 63 & 7.0 & $\mathrm{~N} / \mathrm{A}$ & 210 \\
\hline $\mathrm{PEA}_{2} \mathrm{FA}_{\mathrm{n}-1} \mathrm{Sn}_{\mathrm{n}} \mathrm{I}_{3 \mathrm{n}+1}$ & $\mathrm{LiF}$ & 0.47 & 20.1 & 74 & 7.0 & $\begin{array}{c}\text { Shelf life, } \mathrm{N}_{2}, \\
5 \mathrm{~d}(90 \%)\end{array}$ & 211 \\
\hline $\mathrm{MASnI}_{3}$ & $\mathrm{~m}-\mathrm{TiO}_{2}$ & 0.88 & 16.8 & 42 & 6.4 & $\mathrm{~N} / \mathrm{A}$ & 17 \\
\hline $\mathrm{PEA}_{0.15} \mathrm{FA}_{0.85} \mathrm{SnI}_{3}$ & indene- $\mathrm{C}_{60}$ & 0.94 & 17.4 & 75 & 12.4 & $\begin{array}{l}\text { Shelf life, } N_{2}, \\
3800 \text { h }(90 \%)\end{array}$ & 21 \\
\hline $\mathrm{FASnI}_{2} \mathrm{Br}$ & $\mathrm{C}_{60}$ & 0.47 & 6.8 & 54 & 1.7 & $\begin{array}{c}\text { Shelf life, } \mathrm{N}_{2}, \\
\text { Degraded in } \\
\text { few hours }\end{array}$ & 169 \\
\hline
\end{tabular}




\begin{tabular}{cccccccc}
$\mathrm{FASnI}_{3}$ & $\mathrm{TiO}_{2}-\mathrm{ZnS}$ & 0.38 & 23.1 & 60 & 5.3 & $\mathrm{~N} / \mathrm{A}$ & 201 \\
$\mathrm{MASn}_{0.5} \mathrm{~Pb}_{0.5} \mathrm{I}_{3}$ & $\begin{array}{c}\text { Fullerene } \\
\text { Derivatives }\end{array}$ & 0.69 & 22.8 & 65 & 10.2 & $\mathrm{~N} / \mathrm{A}$ & 212 \\
$\mathrm{FA}_{0.5} \mathrm{MA}_{0.5}$ & $\mathrm{PC}_{61} \mathrm{BM}$ & 0.75 & 30.6 & 76 & 17.6 & $\mathrm{~N} / \mathrm{A}$ & 214 \\
$\mathrm{Sn}_{0.5} \mathrm{~Pb}_{0.5} \mathrm{I}_{3}$ & & & & & & & \\
\hline
\end{tabular}

Note: $\mathrm{LDP}=$ low dimensional perovskites, $\mathrm{PEG}=$ polyethylene glycol.

Hole-Transporting Layer (HTL): Despite the compatible band alignment of Spiro-OMeTAD with Sn-PVSKs, an additional oxidation step is needed to obtain high electrical conductivity, which place the underlying Sn-PVSK active layer at a risk of oxidation. Although alternate holetransporting material such as poly[bis(4-phenyl) (2,4,6-trimethylphenyl)amine] (PTAA) was implemented, ${ }^{40,71,73,202}$ the normal device configuration may suffer electron-deficient environment at the utmost surface of devices under operation if the holes are not effectively extracted, which makes the $\mathrm{Sn}^{2+}$ adjacent to HTL easy to oxidize. Therefore, most of the highly-efficient Sn-PSCs were fabricated with the inverted structure. However, the commonly used PEDOT:PSS HTL exhibits problematic issues such as hygroscopic nature, strong acidity, irreversible reactivity and imperfect match with Sn-PVSKs, which lead to deteriorated device performance and stability in the devices.

To circumvent these issues, some research groups managed to find alternatives for the PEDOT:PSS layer. ${ }^{203-207}$ For instance, Sun and coworkers applied p-type $\mathrm{NiO}_{\mathrm{x}}$ with a suitable energy level for efficient hole extraction from $\mathrm{CsSnI}_{3}$, which delivered a promising PCE of 3.31\% in the absence of any additive. ${ }^{203}$ In another work, PCP-Na (Figure 14A) was introduced between PEDOT:PSS and $\mathrm{FASn}_{0.5} \mathrm{~Pb}_{0.5} \mathrm{I}_{3}$ layers to facilitate hole extraction for its high $\mathrm{HOMO}$ energy level as shown in Figure 14B, leading to hysteresis-free $J-V$ characteristics as shown in Figure 14C. 205

Other groups sought to overcome the abovementioned issues in PEDOT:PSS by chemical modification $^{208,209}$ or inserting buffer layer. ${ }^{210,211}$ For example, the Han's group intercalated 
PEDOT:PSS with PEG molecule to minimize the mismatch of band alignment between $\mathrm{FASnI}_{3}$ and HTL, which increased the PCE from $2.01 \%$ to $5.12 \%$ as shown in Figure 14D-F. ${ }^{208}$ The Li team improved the humidity tolerance of devices by blending PEDOT:PSS with hydrophobic PBDB-T:ITIC, which further passivated the trap states and dramatically decreased the device $\mathrm{E}_{\text {loss }}$ below $0.4 \mathrm{eV} .{ }^{209}$ As for buffer layer, both the $\mathrm{Zhu}$ and $\mathrm{Wu}$ groups introduced a thin 2D layer between the Sn-PVSKs and HTL, which reduced both crystal lattice and energy level mismatch and delivered outstanding PCEs of $7.05 \%$ and $6.98 \%$, respectively. ${ }^{210,211}$

Electron-Transporting Layer (ETL): Besides the endeavor to improve the compatibility between HTL and active layer, there were also many researches on tuning the properties of ETL. aiming at minimizing energy level mismatches and suppressing the ion diffusion. ${ }^{202,212-217}$

Inherited directly from normal structure of $\mathrm{Pb}-\mathrm{PSCs}$, the utilization of c-TiO $2 / \mathrm{m}-\mathrm{TiO}_{2} \mathrm{ETL}$ was firstly used in $\mathrm{MASnI}_{3}$ by the Snaith group and realized a promising PCE over $6 \%$ for PVSK solar cell. ${ }^{17}$ In order to minimize the energy level mismatch, the Kanatzidis group modified $\mathrm{TiO}_{2}$ with $\mathrm{ZnS}$ to form a cascade energy level landscape, which suppressed interfacial recombination and promoted electron transfer. As a result, the devices exhibited a decent PCE of 5.27\% with an improved $V_{\mathrm{OC}}$ of $0.380 \mathrm{~V} \cdot{ }^{202}$ On the other hand, fullerene and its derivatives are commonly used in the inverted device configuration. Guo and coworkers systematically compared different kinds of fullerene derivatives as ETL for MASn ${ }_{0.5} \mathrm{~Pb}_{0.5} \mathrm{I}_{3}$ based PSCs (Figure $14 \mathrm{G}-\mathrm{I}$ ) and then found the lowest unoccupied molecular orbit (LUMO) energy level of ETL critically affect to acquire high $V_{\mathrm{OC}} \cdot{ }^{212}$ Consistently, Hatton et al. also reported that the $V_{\mathrm{OC}}$ loss in $\mathrm{CsSnI}_{3}$ based PSCs decreased with increasing LUMO level, which reduced the electron energy loss upon its extraction into the ETL. ${ }^{213}$ Inspired by highly efficient $\mathrm{Cu}(\mathrm{In}, \mathrm{Ga}) \mathrm{Se}_{2}$ solar cells, Kapil and coworkers introduced a thin buffer layer of $\mathrm{PC}_{61} \mathrm{BM}$ between the $\mathrm{C}_{60}$ and active layer to realize a spike structure which 
efficiently suppresses interfacial recombination and maximized the $V_{\mathrm{OC}}$ up to $0.5 \mathrm{~V}$. Consequently, the champion devices achieved a superior PCE of $17.6 \% .^{214}$
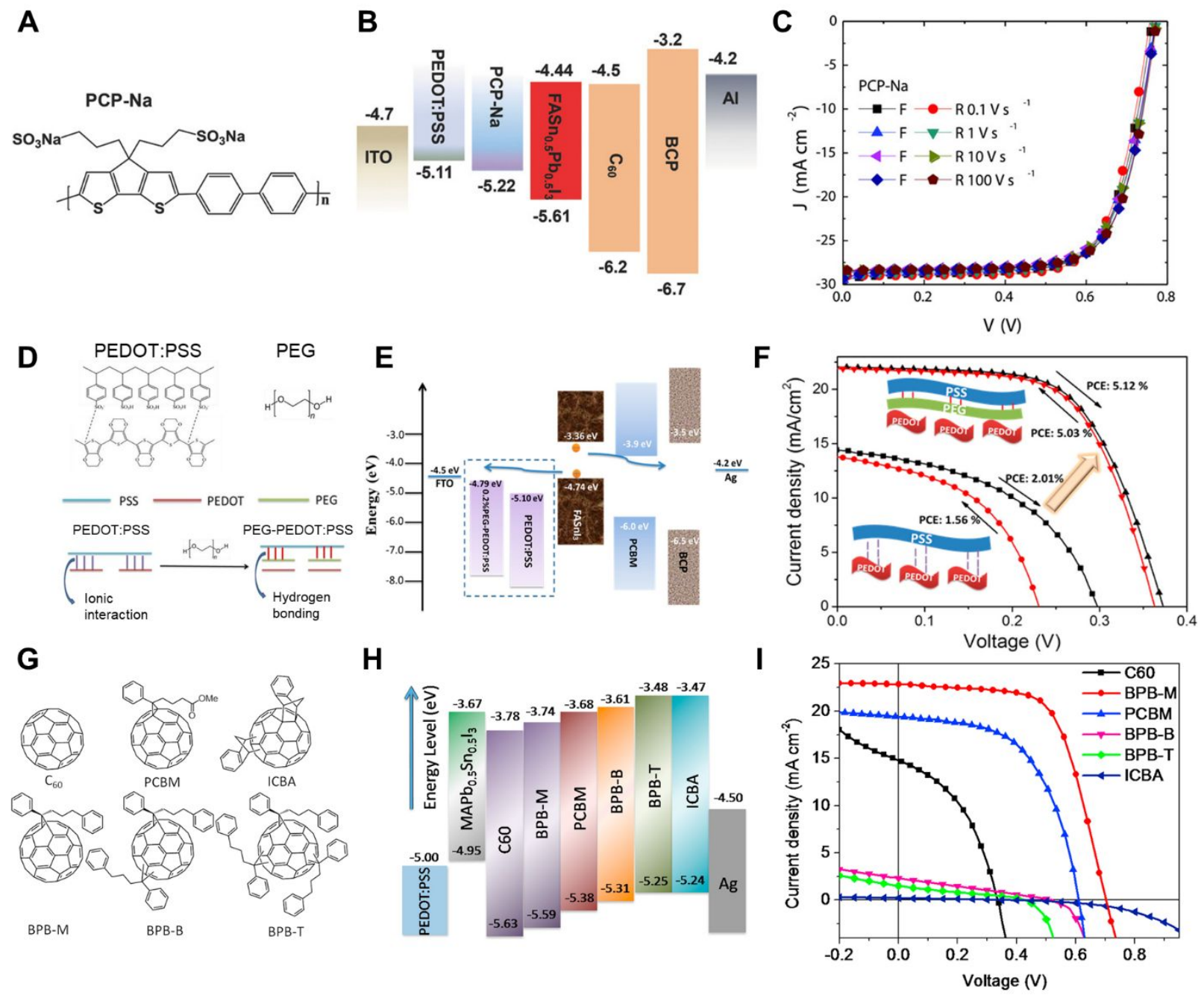

Figure 14. (A) Chemical structure of PCP-Na. (B) Schematic diagram of the energy levels of the various layers used in the device structure. (C) J-V curves of the champion device fabricated with PCP-Na as HEL tested with different sweep rates. (A-C) Reprinted with permission from Ref. 205. Copyright 2019, Wiley-VCH. (D) Molecular structures of PEDOT:PSS and PEG together with schematic illustration of a possible mechanism for VBM changes induced by intercalation of PEG into PEDOT:PSS. (E) energy diagram for devices with this configuration. (F) $\mathrm{J}-\mathrm{V}$ curves and for $\mathrm{FASnI}_{3}$ PSCs based on PEDOT:PSS and PEG-PEDOT:PSS. (D-F) Reprinted with permission from Ref. 208. Copyright 2019, American Chemical Society. (G) Chemical structures of the electron transport materials $\left(\mathrm{C}_{60}\right.$ and fullerene derivatives and $\mathbf{( H )}$ energy diagram in real device. (I) $\mathrm{J}-\mathrm{V}$ curves of $\mathrm{MASn}_{0.5} \mathrm{~Pb}_{0.5} \mathrm{I}_{3}$ PSCs with the scan-rate of $50 \mathrm{mV} \mathrm{s}^{-1}$. (G-I) Reprinted with permission from Ref. 212. Copyright 2019, Elsevier.

Phase/Structure Tailoring: In order to enhance structural stability along with charge transport 
in Sn-PVSKs. Researchers sought to adopt strategies such as lattice structure tailoring (e.g., 3D hollow structure and LD-PVSKs) and phase component regulation (i.e., heterojunctions), both of which have demonstrated feasible to mitigate the controversy between device reliability and performance. In this part, we will focus on three approaches towards modulating the phase/structure of Sn-PVSKs. The detailed device performance and stability parameters are summarized in Table 5.

Table 5. Summary of device performance and stability of Sn-PSCs by the phase and structure regulation strategies

\begin{tabular}{|c|c|c|c|c|c|c|c|}
\hline Active Layer & Strategy & $\begin{array}{l}V_{\text {OC }} \\
(\mathrm{V}) \\
\end{array}$ & $\begin{array}{c}J_{\mathrm{SC}} \\
\left(\mathrm{mA} / \mathrm{cm}^{2}\right) \\
\end{array}$ & $\begin{array}{l}\text { FF } \\
(\%)\end{array}$ & $\begin{array}{l}\text { PCE } \\
(\%)\end{array}$ & $\begin{array}{l}\text { Stability } \\
\text { Test (r.t.) }\end{array}$ & Ref. \\
\hline$\{\mathrm{en}\} \mathrm{MASnI}_{3}$ & & 0.43 & 24.3 & 63.7 & 6.6 & $\begin{array}{l}\text { Continuous } \\
\text { operation, } \\
\text { Air, } 10 \mathrm{~min} \\
\quad(60 \%)\end{array}$ & 71 \\
\hline$\{$ en $\} \mathrm{FASnI}_{3}$ & Hollow & 0.48 & 22.5 & 66.0 & 7.1 & $\begin{array}{c}\text { Shelf life, } \\
\text { encapsulated, } \\
1000 \text { h }(96 \%)\end{array}$ & 72 \\
\hline $\mathrm{PN}_{-F A S n I}$ & 3D PVSKs & 0.43 & 22.2 & 60.7 & 5.8 & N/A & 73 \\
\hline $\mathrm{HEA}_{0.4} \mathrm{FA}_{0.6} \mathrm{SnI}_{3}$ & & 0.37 & 18.5 & 56.0 & 3.9 & $\begin{array}{c}\text { Shelf life, } \mathrm{N}_{2}, \\
1000 \mathrm{~h} \\
(100 \%)\end{array}$ & 74 \\
\hline $\begin{array}{c}\{\mathrm{en}\} \mathrm{FA}_{0.5} \mathrm{MA}_{0.5} \\
\mathrm{Sn}_{0.5} \mathrm{~Pb}_{0.5} \mathrm{I}_{3}\end{array}$ & & 0.82 & 27.2 & 76.1 & 17.0 & $\begin{array}{c}\text { Shelf life, 10- } \\
30 \% \text { RH, } 2 \text { h } \\
(92 \%)\end{array}$ & 218 \\
\hline $\mathrm{PEA}_{0.15} \mathrm{FA}_{0.85} \mathrm{SnI}_{3}$ & $2 \mathrm{D} / 3 \mathrm{D}$ & 0.61 & 22.0 & 70.1 & 9.4 & $\begin{array}{c}\text { Shelf life, } N_{2}, \\
600 \text { h }(90 \%)\end{array}$ & 88 \\
\hline $\mathrm{PEA}_{2} \mathrm{FA}_{\mathrm{n}-1} \mathrm{Sn}_{\mathrm{n}} \mathrm{I}_{3 \mathrm{n}+1}$ & $\begin{array}{l}\text { heterojunc } \\
\text { tions }\end{array}$ & 0.52 & 24.1 & 71.0 & 9.0 & $\begin{array}{c}\text { Shelf life, } \\
\text { 20\% RH, } 76 \\
\text { h }(59 \%)\end{array}$ & 230 \\
\hline $\mathrm{BA}_{2} \mathrm{MA}_{3} \mathrm{Sn}_{4} \mathrm{I}_{13}$ & $\begin{array}{l}\text { 2D RP } \\
\text { PVSKs }\end{array}$ & 0.38 & 21.87 & 48.3 & 4.0 & $\begin{array}{c}\text { Shelf life, } N_{2}, \\
94 \text { d }(100 \%)\end{array}$ & 79 \\
\hline
\end{tabular}


Note: $\{\mathrm{en}\}=$ ethylene diammonium, $\mathrm{PN}=$ propylene diammonium, $\mathrm{HEA}=2$ hydroxyethylammonium, t-BA = tert-butylammonium, $\mathrm{Bn}=$ benzimidazolium, 4AMP $=4$ (aminomethyl)piperidinium, $\mathrm{HA}=$ hydrazinium, $\mathrm{NCs}=$ nanocrystals, $\mathrm{RP}=$ Ruddlesden-Popper, DJ = Dion-Jacobson.

3D Hollow Structures: The 3D hollow-structured have demonstrated as promising lightabsorbing materials because of their flexible $E_{\mathrm{g}}$ tunability as well as the improved ambient tolerance. ${ }^{72}$ Typically, the hollow $3 \mathrm{D}$ structure derives from conventional $\mathrm{ABX}_{3}$ with neutral fragments of $\mathrm{SnI}_{2}$ vacancies substituted by moderate sized organic cations. The interconnected 3D $\left[\mathrm{BX}_{6}\right]^{4-}$ framework can be partially replaced by moderate sized diamine cations such as ethylene diammonium ( $\{\mathrm{en}\}$, also referred as EDA as mentioned in previous sections), ${ }^{71,72,218}$ propylene diammonium $(\mathrm{PN})$ and trimethylene diammonium $(\mathrm{TN})^{73}$ without disturbing the dimensionality as displayed in Figure 15A. The resultant structure features massive $\mathrm{B}$ and $\mathrm{X}$ site vacancies accompanied by a blue-shift of $E_{\mathrm{g}}$ from 1.25 to $1.51 \mathrm{eV}$ owing to adjustable orbital overlaps between $\mathrm{Sn} / \mathrm{I}{ }^{219}$ Besides, the air stability is dramatically enhanced in comparison to the FA and MA based reference devices, which originates from the downshift of VBM induced by $\mathrm{SnI}_{2}$ 
vacancies.

In 2017, the Kanatzidis group for the first time introduced $\{$ en $\}$ in replacement of $\mathrm{MA}^{+}$or $\mathrm{FA}^{+}$ to generate the so-called 3D hollow structure for Pb-PSCs. ${ }^{71,72}$ As mentioned above, the $\{\mathrm{en}\}$ manifests an efficient crystallization regulator by forming an adduct with the Sn-PVSK lattices, which significantly improves film morphology. Besides, an incorporation of $\{$ en $\}$ partially replaces the neutral $\mathrm{SnI}_{2}$ unit and generate vacancies in the 3D Sn-PVSK lattices, which reduces the average length of the remaining $\mathrm{Sn}-\mathrm{I}$ bonds and lowers the position of CBM and VBM, leading to a larger $E_{\mathrm{g}}$ and oxidation-resistant lattices in Sn-PVSKs. Afterwards, Diau et al. realized the same structure by introducing bifunctional 2-hydroxyethylammonium (HEA) into FASnI 3 . Such structural doping gradually increases the crystallographic symmetry from orthorhombic $(x=0)$, rhombohedral $(0.2 \leq \mathrm{x} \leq 0.4)$, tetragonal 3D-vacant structure $(0.6 \leq \mathrm{x}<1)$ (Figure $15 \mathrm{~B})$ to the kinetically preferable $\mathrm{HEASn}{ }_{0.67} \mathrm{I}_{2.33}$ and thermodynamically preferable $\mathrm{HEA}_{2} \mathrm{SnI}_{4}$ with complete $\mathrm{HEA}^{+}$replacement (Figure 15C). ${ }^{74}$ Moreover, they stressed that this 3D-vacant perovskite bridged 2D and 3D perovskites, which indicates its potential to achieve both high ambient stability and device performance. Later, the Kanatzidis group continued to enrich the diversity of 3D hollow PVSKs by discovering two new kinds of diammonium-PN and TN, followed by their incorporation into $\mathrm{FASnI}_{3}$, which exhibited reduced leakage current and lower trap-state density. As a result, a $10 \mathrm{~mol} \%$ addition of $\mathrm{PN}$ and $\mathrm{TN}$ enabled highly efficient devices with promising PCEs of $5.85 \%$ and $5.53 \%$, respectively. ${ }^{73}$ Most recently, they extended the universality of hollow structure to $\mathrm{Pb} / \mathrm{Sn}$ alloyed system by loading $5 \mathrm{~mol} \%$ en $\}$ into $\mathrm{FA}_{0.5} \mathrm{MA}_{0.5} \mathrm{Sn}_{0.5} \mathrm{~Pb}_{0.5} \mathrm{I}_{3}$, which obtained a better match between the HTL and the active layer and facilitated charge transport. The as-fabricated device exhibited enhanced ambient stability and an outstanding PCE of 17.04\%, which holds good promise of 3D hollow structure in achieving ideal- $E_{\mathrm{g}}$ materials with better 
chemical stability. ${ }^{218}$

Low-Dimensional Structures: The hollowed 3D structure can be viewed as the transition state between 2D and 3D PVSKs, which will transform to lower dimensionality if larger ligands are introduced. As previously discussed, the LD-PVSKs are symbolic of layered $\left[\mathrm{BX}_{6}\right]^{4-}$ octahedra sandwiched between or surrounded by hydrophobic organic spacers, which remarkably improve the ambient tolerance of PVSKs lattices..$^{89,220-222}$ However, the increasing quantum confinement effect with a decreasing dimensionality results in larger binding energy $\left(E_{\mathrm{b}}\right)$ and $E_{\mathrm{g}} \mathrm{s}$, which makes difficult exciton splitting and limits the absorption range. Therefore, most photovoltaic applications regarding Sn-based LD-PVSKs are based on 2D layered structure with moderate $E_{\mathrm{b}}$ and $E_{\mathrm{g}}$. On the other hand, the insulating nature of organic spacer significantly blocks charge transport in thin films. Therefore, various strategies of end-groups functionalization and structural tailoring in organic spacers were proposed and added great synthetic and structural versatility to the Sn-based 2D-PVSKs. ${ }^{89,220,223-229}$

As the initial attempt, the Ning group demonstrated the first photovoltaic application of 2D $(\mathrm{PEA})_{2}(\mathrm{FA})_{\mathrm{n}-1} \mathrm{Sn}_{\mathrm{n}} \mathrm{I}_{3 \mathrm{n}+1}$ and found the crystal orientation of $2 \mathrm{D}$ domains varied with the PEA content, which exhibited the highest orientation vertical to the substrate with an optimal loading of $20 \mathrm{~mol} \%$ PEA, thereby strikingly improving charge transport in device. ${ }^{225}$ As a result, the $(\mathrm{PEA})_{2}(\mathrm{FA})_{8} \mathrm{Sn}_{9} \mathrm{I}_{28}$ based devices achieved a PCE of 5.94\% with an enhanced stability over $100 \mathrm{~h}$ in inert glovebox compared to the $\mathrm{FASnI}_{3}$ reference device. In order to address the widened $E_{\mathrm{g}} \mathrm{s}$, the Nazeeruddin group presented the synthesis of benzimidazolium (Bn) and benzodiimidazolium (Bdi) based 2D Sn-PVSKs with narrower $E_{\mathrm{g}} \mathrm{s}$ than traditional amino groups, which resulted from the minor distortions caused by symmetrical hydrogen bonding of the imidazolium cations. ${ }^{220}$ As for the hindered charge transport, Padture and coworkers explored the probability of 2D Dion- 
Jacobson (DJ) structured PVSKs by applying a newly discovered 4-(aminomethyl)piperidinium cation $\left(4 \mathrm{AMP}^{+}\right)$as an organic spacer. The resultant layered PVSKs exhibited improved charge transport as a result of the reduced organic content compared to its RP analogues, yielding the best-performing device $(n=4)$ with a PCE of $4.22 \% .^{226}$

Heterojunction Construction: In addition to structure tailoring, introduction of extra phases to form heterojunctions also proved to facilitate exciton separation and charge transport. $88,211,230,231$ The heterojunction provides additional driving force for exciton splitting owing to the energy level differences between and heterogeneous phases. Besides, some components may act as defect passivator or in-situ encapsulation, which improve the ambient stability of Sn-PVSK matrix. In an early attempt, the Loi group introduced a trace amount of PEA based 2D Sn-PVSKs into $\mathrm{FASnI}_{3}$ to enable the homogeneous growth of $3 \mathrm{D}$ phases with high orientation at low temperature as indicated from the crystal structures and GIWAXS profiles in Figure $15 \mathrm{D}-\mathrm{F} .{ }^{230}$ The resulting 2D/3D bulk heterojunctions (BHJs) showed decreasing GBs along with Sn vacancies, which pushed the device performance from $6 \%$ to $9 \%$. Then, Ning et al. demonstrated a gradient $2 \mathrm{D}$ quasi-2D-3D structure in $\mathrm{PEA}_{0.15} \mathrm{FA}_{0.85} \mathrm{SnI}_{3}$. The overlaying 2D PVSKs effectively protected the underlying 3D phases from oxidation. The hierarchical alignment of phases formed cascade energy landscapes for efficient charge transport. Consequently, the device yielded an outstanding PCE up to $9.41 \%$ along with excellent stability, which retained $90 \%$ of its initial performance for almost $600 \mathrm{~h}$ in inert atmosphere. ${ }^{88}$ Besides, Chen and coworkers introduced PTN-Br into GBs of FASnI 3 to construct BHJs. The PTN-Br with compatible VBM not only facilitated hole transport from PVSK crystals to PTN-Br, but also passivated interfacial trap states via the formation of Lewis adduct between uncoordinated $\mathrm{Sn}$ atoms and the di-methylamino group of PTN-Br. As a result, 
the best-performing devices achieved a PCE of $7.94 \%$ with better UV stability, which maintained ca. $66 \%$ of its initial PCE under continuous working operation for 5 h. ${ }^{231}$

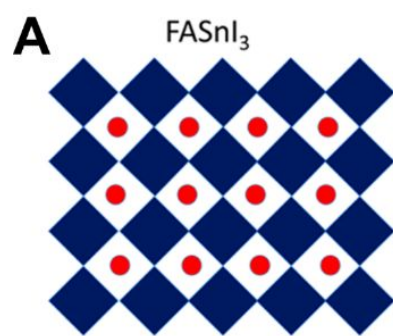

B

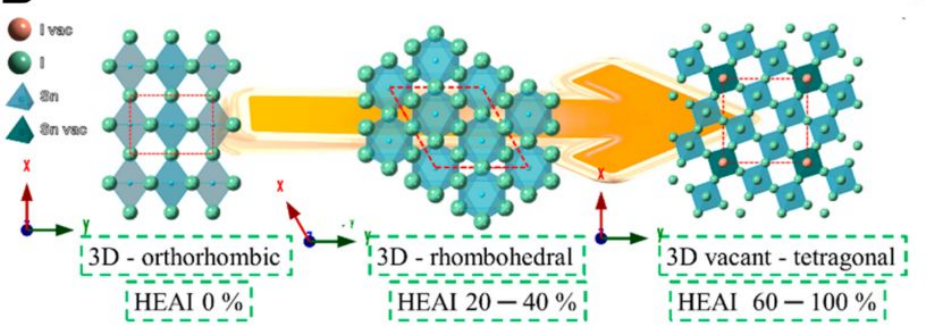

D
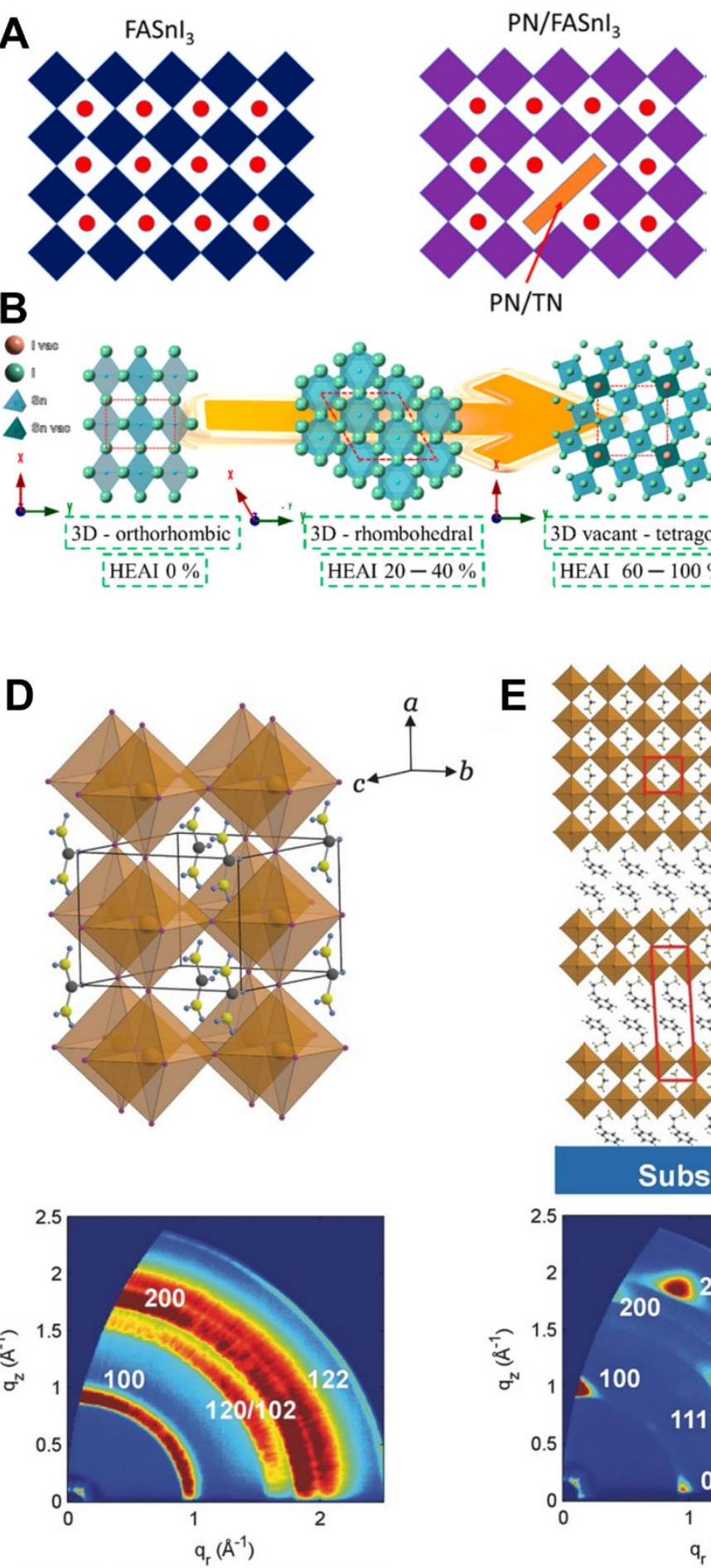

E
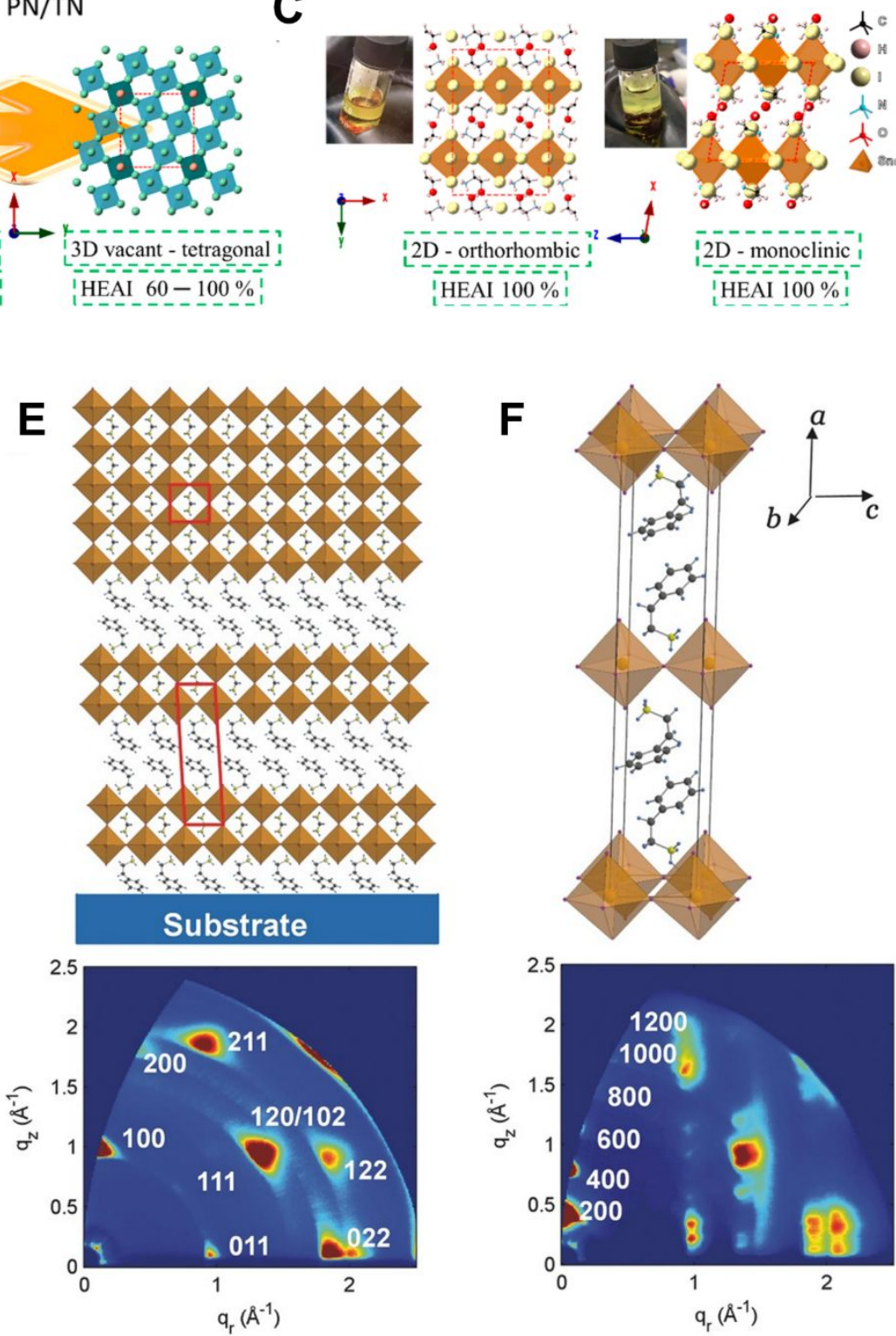

$\mathbf{F}$
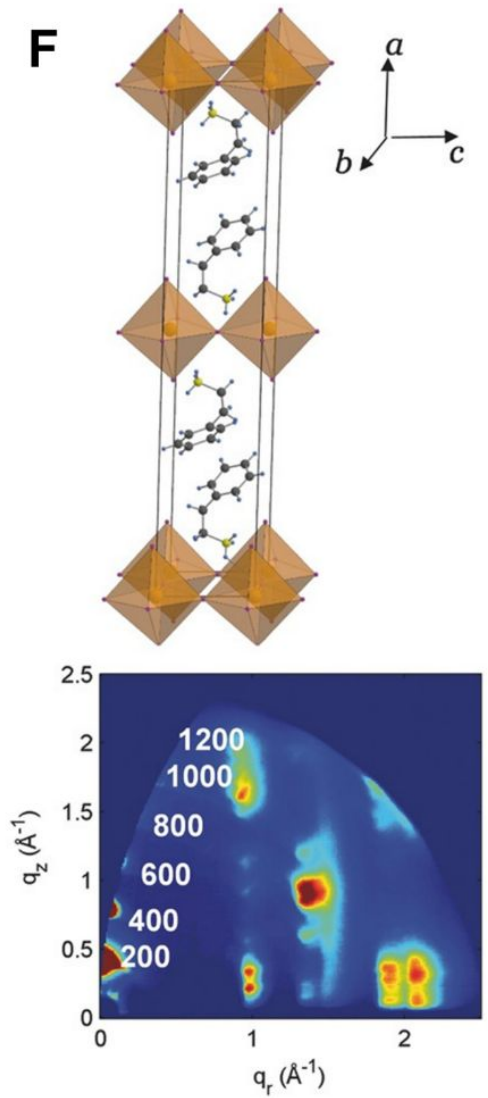

Figure 15. (A) Schematic illustration of the structure of the 3D pristine $\mathrm{FASnI}_{3} \mathrm{PVSK}$ and $3 \mathrm{D}$ hollow $\mathrm{FASnI}_{3}$ with PN or TN. Reprinted with permission from Ref. 73. Copyright 2019, 
American Chemical Society. (B) Kinetic preferred PVSK structures of single crystals with HEAI proportions 0,40 , and $80 \%$ represented from left to right. (C) Thermodynamically preferred crystal structures obtained from two HEAI $100 \%$ single crystals show the 2 D structural features. (B, C) Reprinted with permission from Ref. 74. Copyright 2019, American Chemical Society. Schematic crystal structure of (D) 3D reference $\mathrm{FASnI}_{3}$, (E) 2D/3D mixture and (F) 2D PEA $2 \mathrm{SnI}_{4}$. (D-F) Reprinted with permission from Ref. 230. Copyright 2019, Wiley-VCH.

Light-Emitting Diodes. In contrast to a multitude of efforts in Sn-PSCs, the other two prime optoelectronic functions_-LEDs and lasing seem to catch less attention, which might be ascribed to either the poor device stability or the unsatisfactory performance lagging behind the existing commercialized materials. However, the favorable optoelectronic properties of Sn-PVSKs such as

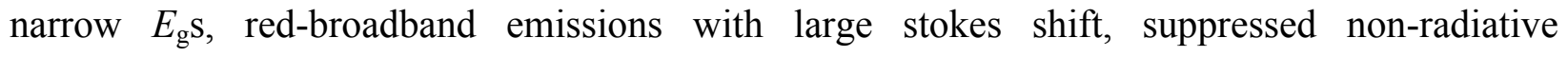
recombination rates along with room-temperature solution processability have shown brighter future than other semiconductor materials such as $\mathrm{GaAs}$, InGaN, organic polymers and colloidal quantum dots (QDs). In addition to the common issues in photovoltaic applications, the optimizing strategy for LED may focus on reducing non-radiative recombination. In this part, we will dive into the highly efficient Sn-based LED applications and discuss about the commonly adopted strategies to improve the device performance as well as stability issues.

As for the additive, The Seok group added elemental sulfur to introduce $\mathrm{S}^{2-}$ into the host lattice alloyed $\mathrm{MAPb}_{0.75} \mathrm{Sn}_{0.25} \mathrm{Br}_{3}$, which lowered the defect concentration by neutralizing the $\mathrm{Sn}^{4+}$, and suppressed defect-assisted non-radiative recombination processes. The S-modified QDs remained phase-stable for more than a month in air conditions. ${ }^{232}$ In another case, Stranks and coworkers employed $\mathrm{ZnI}_{2}$ to promote homogeneous mixing of $\mathrm{Sn}$ and $\mathrm{Pb}$ precursor in the alloyed systems, which decreased the Sn-rich regions that act as oxygen-induced degradation sites and thus improved its resistance to oxygen. ${ }^{233}$ Similar to photovoltaics, $\mathrm{SnF}_{2}$ was applied into $\mathrm{CsSnX}_{3}$ (X $=\mathrm{Br}$ or I) by Sum and coworker to reduce the full width half maximum (FWHM) as observed 
from Figure 16A. Mover, they utilized the wing texture of the butterfly (Figure 16B) to form natural photonic crystals and provided the cavity resonance close to the emission peak of $\mathrm{CsSnI}_{3}$, which realized a single-mode, low-threshold lasing $\left(\sim 15 \mu \mathrm{J} \mathrm{cm}{ }^{-2}\right){ }^{234}$

The B and X-site compositional engineering in Sn-PVSKs proved effective approaches to achieve flexible emission tunability. ${ }^{235-239}$ Liu and coworkers doped the $\mathrm{CsPb}_{1-\mathrm{x}} \mathrm{Sn}_{\mathrm{x}} \mathrm{Br}_{3} \operatorname{system}$ with air-stable $\mathrm{Sn}^{4+}$, which effectively suppressed the tr-ion generation and improved PLQY from 45\% to $83 \%$. The optimal $\mathrm{CsPb}_{0.67} \mathrm{Sn}_{0.33} \mathrm{Br}_{3}$ QDs based LED exhibited bright emission at $517 \mathrm{~nm}$, a luminance of $12,500 \mathrm{~cd} \mathrm{~m}^{-2}$, a current efficiency of $11.63 \mathrm{~cd} \mathrm{~A}^{-1}$, an external quantum efficiency (EQE) of $4.13 \%$, a power efficiency of $6.76 \mathrm{~lm} \mathrm{w}^{-1}$ and a low turn-on voltage of $3.6 \mathrm{~V}$, all of which are the best values among reported Sn-PVSK QDs based LEDs. ${ }^{236}$ Besides B-site modulation, Tan and coworkers demonstrated tunable NIR electroluminescence in $\mathrm{MASn}\left(\mathrm{Br}_{1-\mathrm{x}} \mathrm{I}_{\mathrm{x}}\right)_{3}$ by partial halide substitution, ${ }^{237}$ which led to continuous blue-shifted absorption with increasing Br content, which was also observed by the Haque group in $2 \mathrm{D} \mathrm{PEA}_{2} \mathrm{SnI}_{4-\mathrm{x}} \mathrm{Br}_{\mathrm{x}}$ crystals. ${ }^{239}$

As discussed above, the lattice distortion related STE state may cause broadband emission in Sn based LD-PVSKs, ${ }^{80,84,87,239-241}$ which shows potential in white-lighting LEDs. For example, the Haque group reported the fabrication of highly luminescent 2D PVSK crystals by incorporating PEA cations (Figure 16C). ${ }^{239}$ The emissive excitons in trap states resulted in wide-band emission (Figure 16D), large stokes-shifted emission (Figure 16E) and increasing lifetime with increasing iodine content (Figure 16F). Similarly, Ma and coworkers presented a series of OD structured $\left(\mathrm{C}_{4} \mathrm{~N}_{2} \mathrm{H}_{14} \mathrm{X}\right)_{4} \mathrm{SnX} \mathrm{X}_{6}(\mathrm{X}=\mathrm{Br}, \mathrm{I}),{ }^{240}\left(\mathrm{C}_{9} \mathrm{NH}_{20}\right)_{2} \mathrm{SbCl}_{5},{ }^{240}$ and $\left(\mathrm{C}_{9} \mathrm{NH}_{20}\right)_{2} \mathrm{SnBr}_{4},{ }^{87}$ where the individual metal halide octahedra $\left(\mathrm{SnX}_{6}{ }^{4-}\right)$, quadrangular pyramids $\left(\mathrm{SbX}_{5}{ }^{2-}\right)$ and seesaw-shaped $\left(\mathrm{SnBr}_{4}{ }^{2-}\right)$ were completely isolated. Since such structure underwent pronounced excited state structural deformation and reorganization (similar to FE-to-STE conversion), the 0D structured PVSKs also 
feathered strong stokes-shifted broadband emissions. Although STE emission in Sn-based LDPVSKs results in high photoluminescence quantum yield (PLQY), the broadband emission may not be desirable in those applications demanding high color purity. Based on the positive correlated PLQY and binding energy, Chiu and coworkers modulated quantum confinement in 2D layered PVSKs by varying the dielectric constant and managed to realize narrow band emission with a record-high PLQY of $21 \%{ }^{242}$ Recently, the Kanatzidis group reported a new family of allinorganic Sn-PVSKs and perovskitoids that can be stabilized by the heteroleptic coordination of $\mathrm{Cl}^{-}$and $\mathrm{I}^{-}-\mathrm{Cs}_{2} \mathrm{SnCl}_{2} \mathrm{I}_{2}$ and $\mathrm{Cs}_{2.38} \mathrm{Rb}_{1.62} \mathrm{Sn}_{3} \mathrm{Cl}_{8} \mathrm{I}_{2} \cdot{ }^{243}$ Further replacement of A-site $\mathrm{Cs}^{+}$with smaller-sized $\mathrm{Rb}^{+}$led to drastic phase evolution into to a mixture of low-dimensional compounds. The as-prepared PVSKs possessed wide and direct $E_{\mathrm{g}} \mathrm{s}$ and good oxidation resistivity, which paves a new path toward the realization of optoelectronic devices with heteroleptic-halide PVSKs. 

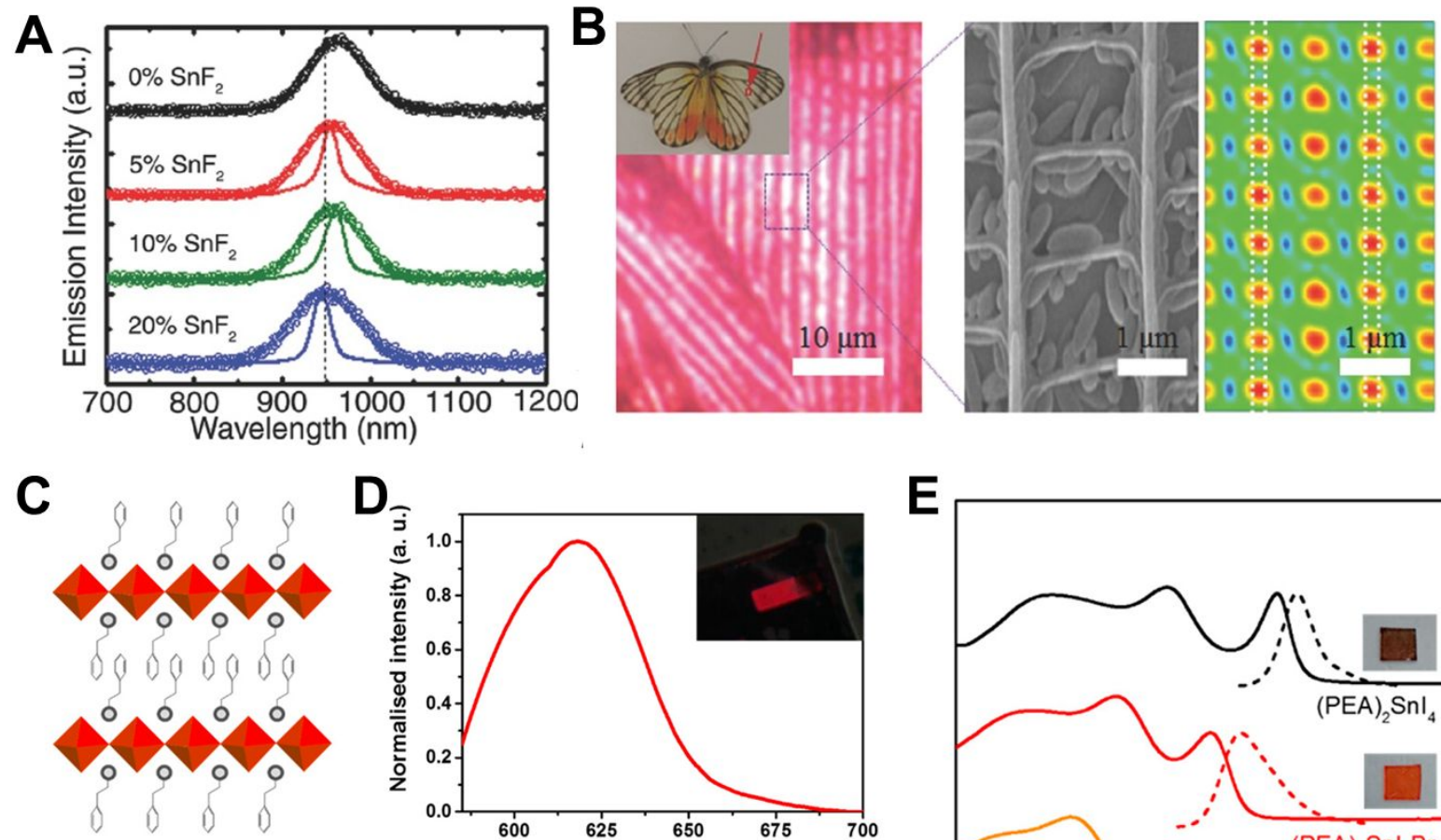

$D_{3}$

$\mathbf{F}$

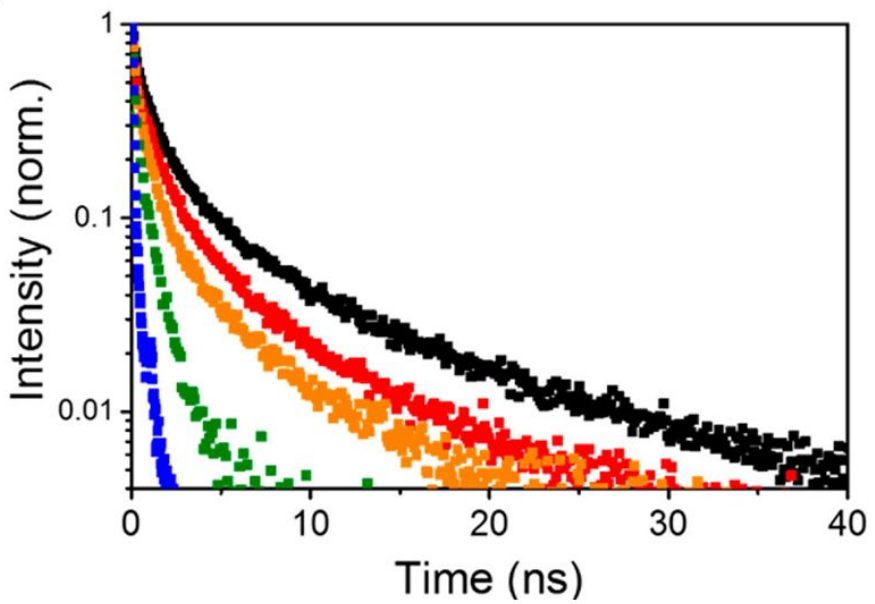

Figure 16. (A) Normalized emission at low pump fluence (open circles, $\approx 1 \mu \mathrm{J} \mathrm{cm}{ }^{-2}$ ) and high pump fluence (solid lines, $\approx 70 \mu \mathrm{J} \mathrm{cm}^{-2}$ ) for $\mathrm{CsSnI}_{3}$ with different molar concentrations of $\mathrm{SnF}_{2}$ treatment. (B) Optical image of a butterfly scale from the white part of the wing. Inset is a photograph of the butterfly. The right panel shows the SEM image of vertical lamellae structure in the scale. (A, B) Reprinted with permission from Ref. 234. Copyright 2019, Wiley-VCH. (C) General crystal schematic of a $(\mathrm{PEA})_{2} \mathrm{SnI}_{\mathrm{x}} \mathrm{Br}_{4-\mathrm{x}} \mathrm{PVSK}$ (light gray balls: $-\mathrm{NH}_{3}{ }^{+}$groups; red polyhedra: corner-sharing $\mathrm{SnX}_{6}{ }^{4-}$ octahedra, where $\mathrm{X}$ represents a halide) with alternating organic-inorganic layers. (D) Electroluminescence spectrum of a (PEA) $)_{2} \mathrm{SnI}_{4}$ device at a bias of 4 V. Inset: the photograph of the LED under operation. (E) Normalized absorbance and PL spectra together with (F) TRPL kinetics (excited at $404 \mathrm{~nm}$ ) of (PEA) ${ }_{2} \mathrm{SnI}_{4}$ (black), (PEA) $\mathrm{SnI}_{3} \mathrm{Br}$ (red), (PEA) ${ }_{2} \mathrm{SnI}_{2} \mathrm{Br}_{2}$ (orange), (PEA) $\mathrm{SnIBr}_{3}$ (green) and (PEA) ${ }_{2} \mathrm{SnBr}_{4}$ (blue) PVSK thin films processed on glass. (C-F) Reprinted with permission from Ref. 239. Copyright 2019, American Chemical Society. Further permissions related to the material excerpted should be directed to the 
ACS.

Photodetectors. Giving the promising optical and electrical properties of Sn-PVSKs, they are also potential building blocks for the application of photodetectors. However, the PD applications differ from photovoltaics in designing purpose and requirements. For example, solar cells are expected to absorb light as wide as possible whereas PDs require selective photo-sensitivity. On the other hand, a large active area is favored in photovoltaic cells while it is necessary to minimize the size of PDs to eliminate the junction capacitance. Nevertheless, the influences of charge carrier dynamics and photo-stability on both devices are similar, which provides analogous avenues for the optimization in PDs.

Recently, the Choy group realized low-temperature and solution-processed Sn-rich PVSK PDs by incorporation of $\mathrm{Rb}^{+}$into A-site cation, which enabled the tunable photodetection from UV to NIR range. ${ }^{244}$ The partial replacement of $\mathrm{MA}^{+}$with $\mathrm{Rb}^{+}$promoted the preferable growth of (110) plane with a lower surface energy, which remarkably ameliorated the film morphology. Consequently, the optimal $\mathrm{Rb}_{0.1} \mathrm{MA}_{0.9} \mathrm{Sn}_{0.65} \mathrm{~Pb}_{0.35} \mathrm{I}_{3}$-based PDs achieved both large photocurrent and low noise current along with an outstanding linear dynamic range of 110 and -3-dB cut-off frequency of $1 \mathrm{MHz}$. For the phase regulation strategies, ${ }^{245-249}$ the Fan group reported the first preparation of $\mathrm{MASnI}_{3}$ PVSK nanowire arrays in nano-engineering templates as shown in Figure $17 \mathrm{~A}-\mathrm{E}{ }^{248}$ The alumina template successfully blocked the incursion of water and oxygen molecules, which efficiently suppressed the aggregation of nanowire. As a result, the phase stability of the embedded nanowires was dramatically enhanced by 840 -fold in comparison to the reference films. To build up heterojunctions, Noh and coworkers coupled the $2 \mathrm{D}(\mathrm{PEA})_{2} \mathrm{SnI}_{4}$ with conjugated polymer wrapped semi-CNTs to form BHJs as shown in Figure $17 \mathrm{~F}-\mathrm{H}$, wherein the semi-CNTs provided extra pathways for efficient carrier transport. ${ }^{246}$ Consequently, the 
$(\mathrm{PEA})_{2} \mathrm{SnI}_{4} /$ semi-CNTs hybrid phototransistors exhibited an ultrahigh photoresponsivity of $6.3 \times$ $10^{4} \mathrm{~A} / \mathrm{W}$ with excellent operational stability compared to reference neat (PEA) $)_{2} \mathrm{SnI}_{4}$ based devices as shown in Figure 17I.
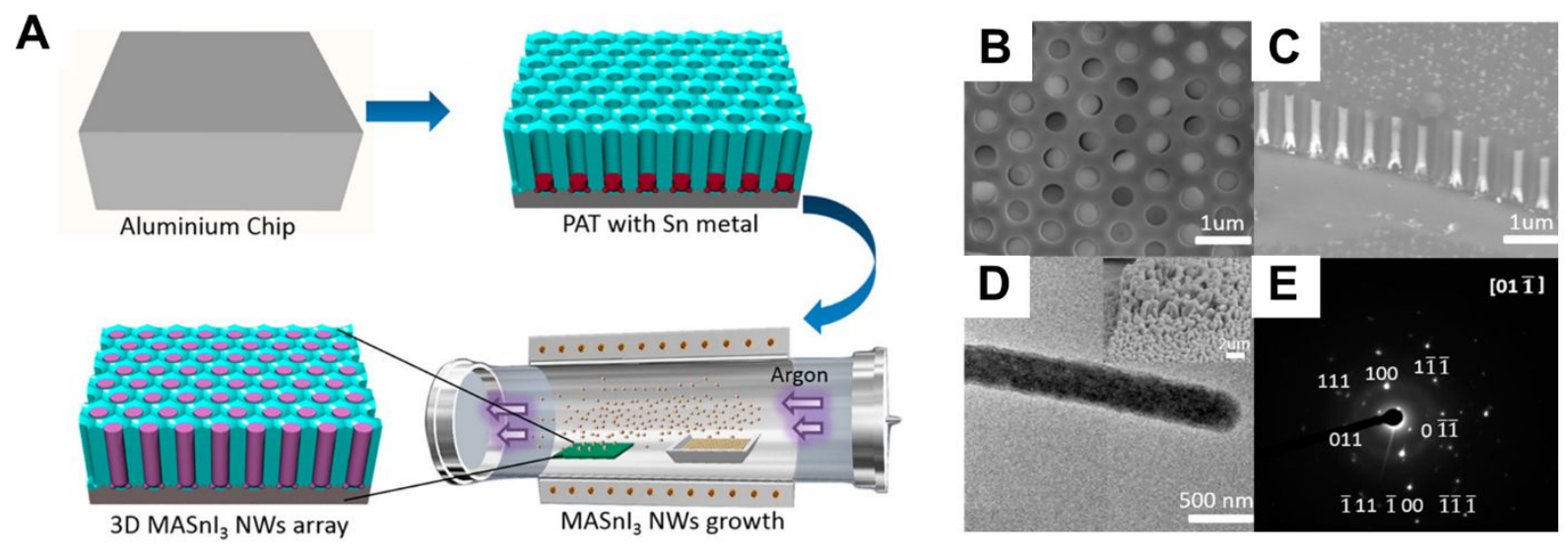

$\mathbf{F}$

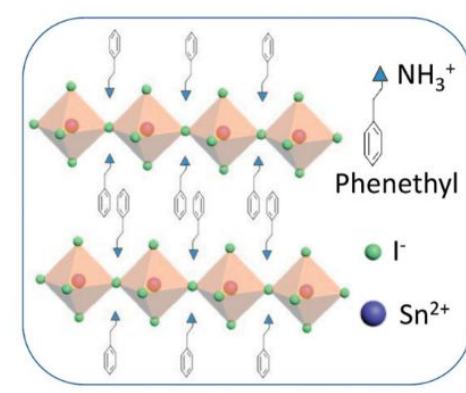

Semi-CNT

suspension
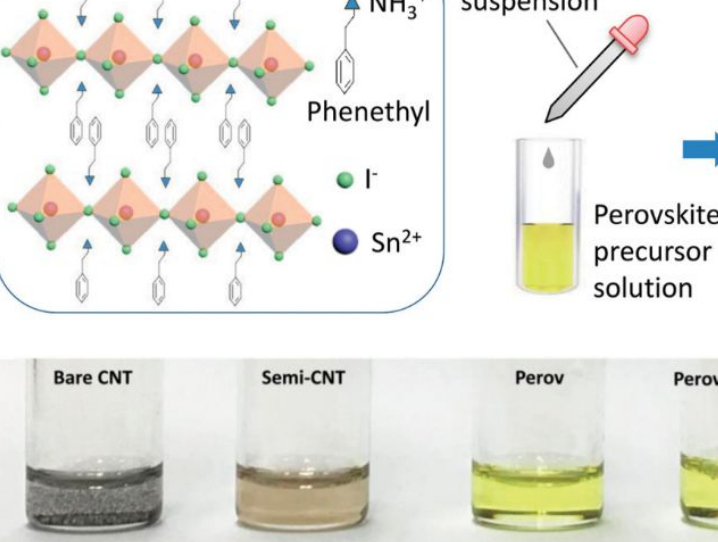

H

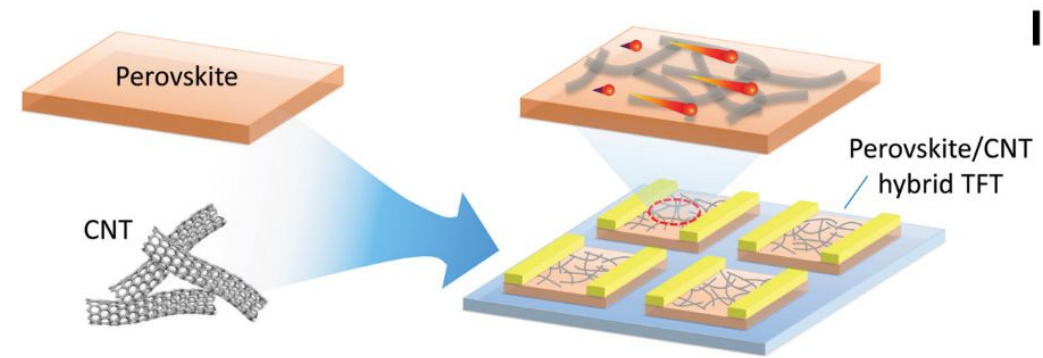

G

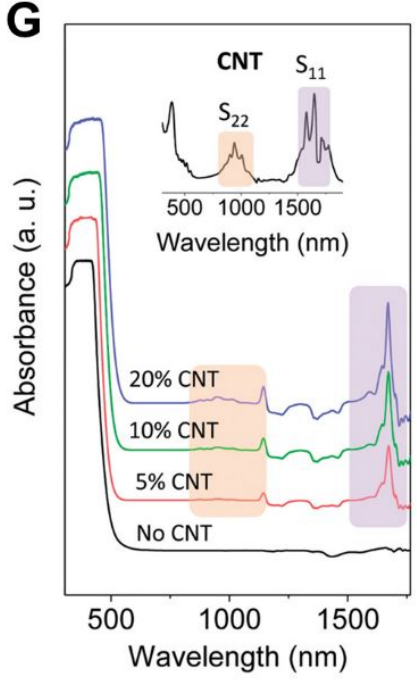

I

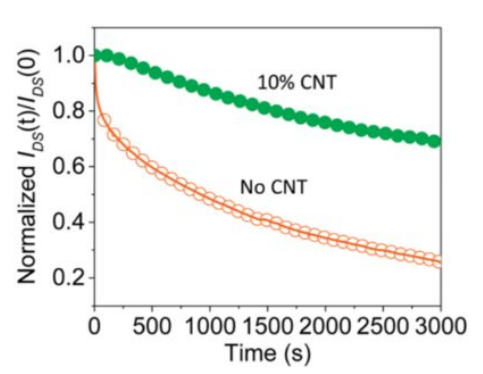

Figure 17. (A) The schematic illustration of the overall growth process for $\mathrm{MASnI}_{3} \mathrm{NWs}$ in a porous alumina template (PAT). (B, C) top and cross-sectional view of SEM image for Sn based PVSK NWs inside PAT. (D) TEM image of a single NW. Inset: SEM image of NWs grown out of nanopores of PAT. (E) Selective area electron diffraction (SAED) pattern for $\mathrm{MASnI}_{3} \mathrm{NWs}$ (A-E) Reprinted with permission from Ref. 248. Copyright 2019, American Chemical Society. 


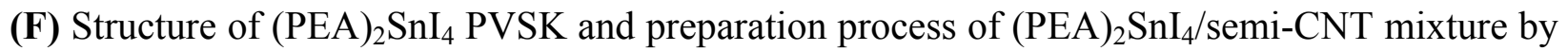
bath sonication. The photographs below show the suspensions/solutions of bare CNTs, polymer wrapped semi-CNTs, PVSKs, PVSKs and semi-CNTs along with PVSK and bare CNTs, respectively (from left to right). (G) Absorption spectra of (PEA) ${ }_{2} \mathrm{SnI}_{4}$ precursor $\mathrm{w}$ and w/o semiCNTs. Inset: Enlarged scale suggesting the absorption of semi-CNTs. (H) Schematic fabrication process and (I) Stability test of (PEA) $)_{2} \mathrm{SnI}_{4}$ based photo-transistor w/ and w/o semi-CNTs. (F-I) Reprinted with permission from Ref. 246. Copyright 2019, American Chemical Society.

Other Applications. The Sn-PVSKs have further fulfilled its potential in other fields such as lasering, ${ }^{250}$ neuromorphic computing, ${ }^{251,252}$ nanogenerator, ${ }^{253,254}$ hydrogen evolution reaction (HER), ${ }^{255}$ electronic logic gates ${ }^{256}$ and field effect transistor. ${ }^{257}$ For example, Ding and coworkers realized photoelectric synaptic plasticity on the basis of $(\mathrm{PEA})_{2} \mathrm{SnI}_{4}$, which was activated in response to a light stimulus in a neuron-like way. The device exhibited several essential synaptic functions such as short- or long-term plasticity and their mutual transmission based on spike frequency control. ${ }^{251}$ As for laser application, Lee and colleagues proposed a low-cost solvothermal method to dope the cholesteric liquid crystal (CLC) with efficient optical gain medium of $\mathrm{CsSnI}_{3}$ QDs, which combined the advantages of suppressed PL loss caused by the quantum confinement of QDs and amplified PL intensity originating from the band-edge effect of the cholesteric liquid crystal (CLC)-distributed feedback resonator. ${ }^{250}$ The resultant CLC lasing cavities doped with QDs presented low threshold (150 nJ/pulse) and narrow line width $(0.20 \mathrm{~nm})$ coupled with lasing-wavelength tunability and long-term durability under humid ambient atmosphere.

In nanogenerator applications, the Yoon group reported the room-temperature preparation of an air-stable $\mathrm{MASnI}_{3}$ piezoelectric nanogenerator, which delivered an output voltage/current of $\sim 3.8 \mathrm{~V}$ and $0.35 \mu \mathrm{A} \mathrm{cm}^{-2}$, respectively. Further incorporation with porous PVDF led to enhanced output voltage of $\sim 12.0 \mathrm{~V}$ and current density of $\sim 4.0 \mu \mathrm{A} \mathrm{cm}{ }^{-2} .{ }^{254}$ Similarly, Kabra and coworkers employed PVDF to prepare the $\mathrm{FASnI}_{3}$ /PVDF nanocomposites, which boosted the ferroelectricity 
of the compounds and ensured the environmental inertia of $\mathrm{FASnI}_{3}{ }^{253}$ The improved piezoelectric response may also originate from the soft polar optic phonon modes as a consequence of the unique orthorhombic ferroelectric phases of $\mathrm{FASnI}_{3}$, which hinders efficient optic to acoustic phonon conversion and results in significant slow hot carrier cooling as revealed by Loi et al. ${ }^{122}$ Interestingly, Tao and coworkers observed DI water induced a self-heal process from black to yellow phase in $\mathrm{DMASnI}_{3}\left(\mathrm{DMA}=\mathrm{CH}_{3} \mathrm{NH}_{2} \mathrm{CH}_{3}{ }^{+}\right.$) PVSK single crystals, ${ }^{255}$ which suggested its potential in HER application. Consequently, the photo-catalysis devices with a HER rate of 0.64 mmol $\mathrm{h}^{-1}$ and good recycling properties. Such unique self-heal properties may be inspirable to address the stability issues in Sn-based optoelectronic applications.

This review has summarized recent advances in Sn-PVSK optoelectronics towards improving both device performance and durability. Based on the detailed discussion of the underlying mechanisms and various strategies, we argue that the development of highly efficient and stable Sn-PVSKs optoelectronics has evolved well along the following two paths. Firstly, the rationally tailored composition, crystal structure and phase composition guarantee the intrinsic stability of Sn-PVSKs structures. Second, the external factors such as additive, solvent, preparation methods as well as interfacial engineering significantly improve the reproducibility, charge transport and block degradation pathways from the aspect of device fabrication. These two means interact with each other and deserve prudential consideration when jointly employed. According to the classification used in Section 4, we present the following directions toward desirable device performance and durability in Sn-PVSKs based optoelectronics.

Additive: Whatever kind of additive is selected, it should not add to instability of Sn-PVSKs during film preparation. From this point-of-view, it would be better for additives to possess certain bonding affinity, for example, lone pair electrons with $\mathrm{Sn}^{2+}$ so as not to elevate the internal energy 
of the whole system. Besides, the cohesive energy of additives and $\mathrm{Sn} \mathrm{X}_{2}$ bonding need to be carefully considered. Strong interaction between them may restrict $\mathrm{SnI}_{2}$ units from forming the $\mathrm{ASnX}_{3}$ structure. Moreover, if the additive exhibits high electronegativity, the 5 s electrons of $\mathrm{Sn}^{2+}$ may also be taken away, similar to the high-temperature oxidation of $\mathrm{Sn}^{2+}$ in DMSO. ${ }^{176}$ In order to pinpoint appropriate additives, the Hard-Soft-Acid-Base theory may be useful. Since $\mathrm{Sn}^{2+}$ belongs to the borderline acid, it can coordinate with either hard or soft bases, which include a wide range of options, to name a few, $\mathrm{SO}_{3}^{-}$(hard base) and $\mathrm{SCN}^{-}$(soft base). Importantly, the shielding and un-shielding effects may provide additional inspirations. For multivalent ions, the lower valence state favors bonding with sulfur or nitrogen atoms containing groups while the higher favors oxygen. To exemplify it, the coordinated phenanthroline and $\mathrm{Fe}^{2+}$ complex is much more stable than that of $\mathrm{Fe}^{3+}$, which is the opposite to that of citrate and ethylenediaminetetraacetic acid (EDTA).

Solvent: The core role of solvent in device engineering lies in the modulation of crystallization. Analogous to the additives, the Lewis base solvent such as DMSO effectively regulates the crystallization by forming intermediates with $\mathrm{SnI}_{2}$ units. However, the high boiling point and low saturated vapor pressure of DMSO makes it difficult to dissipate upon thermal annealing. The mixed solvent strategy then proves viable to modulate the crystallization kinetics and maintain a fine balance of between precursor solubility, crystallization rates and removability. In order to trigger simultaneous nucleation and homogeneous growth, temporally and spatially sufficient contact with anti-solvent is the crux, which necessitates appropriate the residence time on the substrate during spin-coating. In this scenario, the basic properties of solvents such as miscibility with good solvent, viscosity and volatility all deserve careful considerations. The mixed antisolvent strategy seems a viable approach. Moreover, if the additives (either crystallization 
regulator or antioxidants) can be incorporated in to the antisolvent, undesirable film forming and oxidation during preparation may also be effectively modulated and suppressed.

Alternative Methodologies: Since an alternately effective method requires thermodynamically dominated and elegantly designed preparation, a precise control of the stoichiometry, crystallization process and morphological features should be focused on improve the reproducibility and quality of as-prepared thin films. As an exemplary case, the multi-step deposition process successfully separates the nucleation and growth stages, thus providing additional means to tune the crystallization, which complements the solvent and additive engineering strategies.

Composition Engineering: The approach of compositional engineering takes into effect mainly by structure/phase stabilization and $E_{\mathrm{g}}$ tuning. Therefore, the tolerance factor and octahedral factors need to be satisfied first to ensure the stable existence of PVSK structure. Because the A-site cations with different sizes significantly affect the tolerance factor and the structure diversity of Sn-PVSKs, the size control of A-sites may induce various lattice symmetries, thereby affording extra ways to tailor the optoelectronic properties of Sn-PVSKs. Furthermore, the mixed A-site strategy with Cs incorporation manifests as an effective method to enhance the tolerance of moisture, light and heat. Moreover, A-site cations with unique functions such as $\mathrm{HA}^{+}$ antioxidant may endow the PVSK lattices with great ambient stability, which encourages one to perform functionalization on the A-site cations. As for the B-site cations, the anomalous $E_{\mathrm{g}}$ bowing effect upon $\mathrm{Sn}-\mathrm{Pb}$ alloying extends the absorption range into the NIR region. However, the toxicity issue of $\mathrm{Pb}$ is not completely resolved and this method seems more like an optimization strategy for Pb-PVSKs rather than for Sn-PVSKs. The Ge incorporation might be a promising alternative to enhance both the device performance and durability despite its poor solubility and 
high cost. The mixed X-site strategy is more helpful in LED and PD applications due to its widerange tunability of $E_{\mathrm{g}}$, while in PSCs, it is commonly used to suppress the unwanted phase transition during device operation. Moreover, the pseudo-halide anion may be applied to strengthen the crystallinity and orientation simultaneously, much as it functions in Pb-PVSKs.

Transporting Layer: Careful attention should be placed on the reducing energy level mismatch between transporting and active layers. Given the varied ion radius between $\mathrm{Sn}^{2+}$ and $\mathrm{Pb}^{2+}$, both VBM and CBM of Sn- and Pb-PVSK vary from each other, which necessitates the engineering of extraction layer for optimal electron/hole collection. Based on the aforementioned works, the cascade structure with gradient energy level alignment is firstly suggested. Apart from successful demonstration of $\mathrm{TiO}_{2}-\mathrm{ZnS}$ and PEDOT:PSS-PCP-Na, there still remain a wide range of combination in both ETL (e.g., $\mathrm{TiO}_{2}, \mathrm{PTAA} \mathrm{NiO}_{\mathrm{x}}, \mathrm{PC}_{61} \mathrm{BM}$ and $\mathrm{C}_{60}$ ) and $\mathrm{HTL}$ (e.g., PEDOT:PSS, PCP-Na, PEG and PTAA) materials. Meanwhile, the cascade transporting layer efficiently encapsulate active layer, which improves the stability of devices. Second, chemical functionalization is also suggested to customize the existing transporting material to match the active layer. The high background hole density in Sn-PVSK results in intensive hole diffusion into the extraction layer driven by the Fermi level difference. After the equilibrium between carrier diffusion and drift processes is reached, the resulting built-in potential area will fall mainly onto the transporting layer. According to the depletion approximation, the main part of active layer will be electrically neutral, which lacks driving force for charge carrier separation and drift upon photoexcitation and aggravate geminate recombination because of the stagnant electron-hole pairs. Therefore, both the ETL and HTL may possess high background carrier concentrations with respective types of charge carriers to ensure the full coverage of built-in potential zone on the entire the active layer. 
Phase Regulation: For LD-PVSKs, despite various organic spacers enhance the structural stability against ambient erosion, the concomitant quantum confinement along with insulating nature of organic components significantly increases $E_{\mathrm{B}}$ and deteriorates charge transport along the operational direction of devices. Attentions should be then directed towards introducing functional groups and atoms such as $\mathrm{S}, \mathrm{P}, \mathrm{S}=\mathrm{O}, \mathrm{P}=\mathrm{O}$ and $\mathrm{C}=\mathrm{O}$, which not only ameliorates crystallization as discussed in the sections of solvent and additive, but also assists in constructing artificially designed heterojunctions that are advantageous to exciton separation and charge transport. Aside from small molecules, an incorporation of narrow- $E_{\mathrm{g}}$ conjugated polymers to form either BHJs or planar heterojunctions seems more beneficial to enhance both light absorption and stability of the active layer by side-chain decoration and self-encapsulation effect of polymer. On the other side, the construction of 3D hollow structure realizes the flexible tunability of $E_{\mathrm{g}}$ with the least perturbance to the overall 3D lattices, which ensures comparable charge transport to the parent PVSKs and circumvents the drawbacks of LD-PVSKs. Recently, an unprecedented 2D PVSKs with alternating cation in the interlayer (ACI) emerged as a potential candidate for PbPSCs. These ACI-type 2D compounds adopt structures with a high alternative ordering of $\mathrm{GA}^{+}$ and $\mathrm{MA}^{+}$in the interlayer space, which integrate the favorable characteristics from both DJ and RP archetypes. Just as the 3D-hollow PVSKs, we consider the ACI structure an intermediate state from 2D to 3D PVSKs with a trade-off between charge transport and structural durability, which may exhibit a bright future in the territory of Sn-based optoelectronics.

\section{Conflicts of interest}

There are no conflicts to declare.

\section{Author Biographies}


Yajie Yan achieved his bachelor degree at Nanjing University of Science and Technology in 2017. Then, he pursued graduate study under the supervision of Prof. Ziqi Liang at Fudan University. He is now a Ph.D. candidate focusing mainly on developing highly efficient and ambient stable metal halide perovskite solar cells.

http://www.opv-te.fudan.edu.cn/yajie_yan_zh.html

Tõnu Pullerits obtained his Ph.D. at Tartu University in 1991 and pursued his postdoc work in Free University of Amsterdam, Umea University and Lund University. He is the head of the Chemical Physics Division at Lund University, concentrating on energy transport, charge dynamics, photophysics and coherence multidimensional spectroscopy. http://www.chemphys.lu.se/people/head-of-department/tonu-pullerits/ Kaibo Zheng received his Ph.D. degree at Fudan University in 2010. Then, he joined Prof. Tonu Pullerits's group in Lund University as postdoc fellow. Currently double affiliated by the Technical University of Denmark and Lund University, he is centered on photo-excitation and carrier dynamics of semiconductor quantum dots and perovskites.

http://www.chemphys.lu.se/people/researchers/kaibo-zheng/

https://www.dtu.dk/english/service/phonebook/person?id=130500\&cpid=233304\&tab=2\&qt=dtu publicationquery

Ziqi Liang obtained his Ph.D. at Pennsylvania State University in 2006 and pursued postdoctoral work at the University of Cambridge. In 2008, he joined National Research Energy Laboratory and later became Scientist III. Since 2012, Prof. Liang's group has been focusing on organic/ perovskite photovoltaics and thermoelectrics at Fudan University.

https://mse.fudan.edu.cn/ab/14/c18341a174868/page.htm 


\section{Acknowledgements}

This work was supported by Inter-Governmental International Cooperation Projects of Science and Technology Commission of Shanghai Municipality (STCSM) under grant No. 17520710100 (Z.L.). K.Z. acknowledges the support by Danish Council for Independent Research No. 70260037B and Swedish Research Council No. 2017-05337. The work performed in Lund is supported by Swedish Energy Agency Grant No. 446511-1, Swedish research council, STINT and KAW foundation.

(1) Kojima, A.; Teshima, K.; Shirai Y.; Miyasaka T. Organometal Halide Perovskites as VisibleLight Sensitizers for Photovoltaic Cells. J. Am. Chem. Soc. 2009, 131, 6050-6051.

(2) Grancini, G.; Roldán-Carmona, C.; Zimmermann, I.; Mosconi, E.; Lee, X.; Martineau, D.; Narbey, S.; Oswald, F.; Angelis, F. D.; Graetzel, M.; et al. One-Year Stable Perovskite Solar Cells by 2D/3D Interface Engineering. Nat. Commun. 2017, 8, 15684.

(3) Dohner, E. R.; Jaffe, A.; Bradshaw, L. R.; Karunadasa, H. I. Intrinsic White-Light Emission from Layered Hybrid Perovskites. J. Am. Chem. Soc. 2014, 136, 13154-13157.

(4) Akkerman, Q. A.; Rainò, G.; Kovalenko, M. V.; Manna, L. Genesis, Challenges and Opportunities for Colloidal Lead Halide Perovskite Nanocrystals. Nat. Mater. 2018, 17, 394-405. (5) Fang, Y.; Dong, Q.; Shao, Y.; Yuan, Y.; Huang, J. Highly Narrowband Perovskite SingleCrystal Photodetectors Enabled by Surface-Charge Recombination. Nat. Photon. 2015, 9, 679686.

(6) Goldschmidt, V. M. Die Gesetze der Krystallochemie. Naturwissenschaften 1926, 14, 477485.

(7) Li, C.; Lu, X.; Ding, W.; Feng, L.; Gao, Y.; Guo, Z. Formability of $\mathrm{ABX}_{3}(\mathrm{X}=\mathrm{F}, \mathrm{Cl}, \mathrm{Br}, \mathrm{I})$ Halide Perovskites. Acta Crystallogr., Sect. B: Struct. Sci. 2008, 64, 702-707.

(8) https://www.nrel.gov/pv/assets/pdfs/best-research-cell-efficiencies.20200218.pdf

(9) Lee, B.; Stoumpos, C. C.; Zhou, N.; Hao, F.; Malliakas, C.; Yeh, C.-Y.; Marks, T. J.; Kanatzidis, M. G.; Chang, R. P. H. Air-Stable Molecular Semiconducting Iodosalts for Solar Cell Applications: $\mathrm{Cs}_{2} \mathrm{SnI}_{6}$ as a Hole Conductor. J. Am. Chem. Soc. 2014, 136, 15379-15385. 
(10) Maughan, A. E.; Ganose, A. M.; Bordelon, M. M.; Miller, E. M.; Scanlon, D. O.; Neilson, J. R. Defect Tolerance to Intolerance in the Vacancy-Ordered Double Perovskite Semiconductors $\mathrm{Cs}_{2} \mathrm{SnI}_{6}$ and $\mathrm{Cs}_{2} \mathrm{TeI}_{6}$. J. Am. Chem. Soc. 2016, 138, 8453-8464.

(11) Saparov, B.; Hong, F.; Sun, J.-P.; Duan, H.-S.; Meng, W.; Cameron, S.; Hill, I. G.; Yan, Y.; Mitzi, D. B. Thin-Film Preparation and Characterization of $\mathrm{Cs}_{3} \mathrm{Sb}_{2} \mathrm{I}_{9}$ : A Lead-Free Layered Perovskite Semiconductor. Chem. Mater. 2015, 27, 5622-5632.

(12) Hebig, J.-C.; Kühn, I.; Flohre, J.; Kirchartz, T. Optoelectronic Properties of $\left(\mathrm{CH}_{3} \mathrm{NH}_{3}\right)_{3} \mathrm{Sb}_{2} \mathrm{I}_{9}$ Thin Films for Photovoltaic Applications. ACS Energy Lett. 2016, 1, 309-314.

(13) McClure, E. T.; Ball, M. R.; Windl, W.; Woodward, P. M. $\mathrm{Cs}_{2} \mathrm{AgBiX}_{6}(\mathrm{X}=\mathrm{Br}, \mathrm{Cl})$ : New Visible Light Absorbing, Lead-Free Halide Perovskite Semiconductors. Chem. Mater. 2016, 28, $1348-1354$.

(14) Wei, F.; Deng, Z.; Sun, S.; Xie, F.; Kieslich, G.; Evans, D. M.; Carpenter, M. A.; Bristowe, P. D.; Cheetham, A. K. The Synthesis, Structure and Electronic Properties of a Lead-Free Hybrid Inorganic-Organic Double Perovskite $(\mathrm{MA})_{2} \mathrm{KBiCl}_{6}(\mathrm{MA}=$ Methylammonium). Mater. Horiz. 2016, 3, 328-332.

(15) Filip, M. R.; Giustino, F. Computational Screening of Homovalent Lead Substitution in Organic-Inorganic Halide Perovskites. J. Phys. Chem. C 2016, 120, 166-173.

(16) Hao, F.; Stoumpos, C. C.; Cao, D. H.; Chang, R. P. H.; Kanatzidis, M. G. Lead-Free SolidState Organic-Inorganic Halide Perovskite Solar Cells. Nat. Photon. 2014, 8, 489-494.

(17) Noel, N. K.; Stranks, S. D.; Abate, A.; Wehrenfennig, C.; Guarnera, S.; Haghighirad, A. A.; Sadhanala, A.; Eperon, G. E.; Johnston, M. B.; Petrozza, A. M.; et al. Lead-Free Organic-Inorganic Tin Halide Perovskites for Photovoltaic Applications. Energy Environ. Sci. 2014, 7, 3061-3068.

(18) Nishimura, K.; Kamarudin, M. A.; Hirotani, D.; Hamada, K.; Shen, Q.; Iikubo, S.; Minemoto, T.; Yoshino, K.; Hayase, S. Lead-Free Tin-Halide Perovskite Solar Cells with 13\% Efficiency. Nano Energy 2020, 74, 104858.

(19) Tong, J.; Song, Z.; Kim, D. H.; Chen, X.; Chen, C.; Palmstrom, A. F.; Ndione, P. F.; Reese, M. O.; Dunfield, S. P.; Reid, O. G.; et al. Carrier Lifetimes of $>1 \mathrm{~ms}$ in Sn-Pb Perovskites Enable Efficient All-Perovskite Tandem Solar Cells. Science 2019, 364, 475-479.

(20) Zhou, X.; Zhang, L.; Wang, X.; Liu, C.; Chen, S.; Zhang, M.; Li, X.; Yi, W.; Xu, B. Highly Efficient and Stable GABr-Modified Ideal-Bandgap (1.35 eV) Sn/Pb Perovskite Solar Cells Achieve 20.63\% Efficiency with a Record Small $V_{\mathrm{OC}}$ Deficit of 0.33 V. Adv. Mater. 2020, 32, 
1908107.

(21) Jiang. X.; Wang, F.; Wei, Q.; Li, H.; Shang, Y.; Zhou, W.; Wang, C.; Cheng, P.; Chen, Q.;

Chen, L.; et al. Ultra-High Open-Circuit Voltage of Tin Perovskite Solar Cells via An Electron Transporting Layer Design. Nat Commun 2020, 11, 1245.

(22) Chakraborty, S.; Xie, W.; Mathews, N.; Sherburne, M.; Ahuja, R.; Asta, M.; Mhaisalkar, S. G. Rational Design: A High-Throughput Computational Screening and Experimental Validation Methodology for Lead-Free and Emergent Hybrid Perovskites. ACS Energy Lett. 2017, 2, 837845.

(23) Xiao, Z.; Meng, W.; Wang, J.; Mitzi, D. B.; Yan, Y. Searching for Promising New PerovskiteBased Photovoltaic Absorbers: The Importance of Electronic Dimensionality. Mater. Horiz. 2017, 4, 206-216.

(24) Liang, L.; Gao, P. Lead-Free Hybrid Perovskite Absorbers for Viable Application: Can We Eat the Cake and Have It Too? Adv. Sci. 2018, 5, 1700331.

(25) Khalfin, S.; Bekenstein, Y. Advances in Lead-Free Double Perovskite Nanocrystals, Engineering Band-Gaps and Enhancing Stability through Composition Tunability. Nanoscale 2019, 11, 8665-8679.

(26) Ke, W.; Stoumpos, C. C.; Kanatzidis, M. G. "Unleaded” Perovskites: Status Quo and Future Prospects of Tin-Based Perovskite Solar Cells. Adv. Mater. 2019, 31, 1803230.

(27) Konstantakou, M.; Stergiopoulos, T. A Critical Review on Tin Halide Perovskite Solar Cells. J. Mater. Chem. A 2017, 5, 11518-11549.

(28) Shi, Z.; Guo, J.; Chen, Y.; Li, Q.; Pan, Y.; Zhang, H.; Xia, Y.; Huang, W. Lead-Free OrganicInorganic Hybrid Perovskites for Photovoltaic Applications: Recent Advances and Perspectives. Adv. Mater. 2017, 29, 1605005.

(29) Giustino, F.; Snaith, H. J. Toward Lead-Free Perovskite Solar Cells. ACS Energy Lett. 2016, 1, 1233-1240.

(30) Xiao, Z.; Song, Z.; Yan, Y. From Lead Halide Perovskites to Lead-Free Metal Halide Perovskites and Perovskite Derivatives. Adv. Mater. 2019, 31, 1803792.

(31) Ning, W.; Gao, F. Structural and Functional Diversity in Lead-Free Halide Perovskite Materials. Adv. Mater. 2019, 31, 1900326.

(32) Lyu, M.; Yun, J.-H.; Chen, P.; Hao, M.; Wang, L. Addressing Toxicity of Lead: Progress and Applications of Low-Toxic Metal Halide Perovskites and Their Derivatives. Adv. Mater. 2017, 7, 
1602512.

(33) Chatterjee, S.; Pal, A. J. Influence of Metal Substitution on Hybrid Halide Perovskites: Towards Lead-Free Perovskite Solar Cells. J. Mater. Chem. A 2018, 6, 3793-3823.

(34) Hu, H.; Dong, B.; Zhang, W. Low-Toxic Metal Halide Perovskites: Opportunities and Future Challenges. J. Mater. Chem. A 2017, 5, 11436-11449.

(35) Uribe, J. I.; Ramirez, D.; Osorio-Guillén, J. M.; Osorio, J.; Jaramillo, F. $\mathrm{CH}_{3} \mathrm{NH}_{3} \mathrm{CaI}_{3}$ Perovskite: Synthesis, Characterization, and First-Principles Studies. J. Phys. Chem. C 2016, 120, 16393-16398.

(36) Kieslich, G.; Sun, S.; Cheetham, A. K. An Extended Tolerance Factor Approach for OrganicInorganic Perovskites. Chem. Sci. 2015, 6, 3430-3433.

(37) Rohere, G. S. Structure and Bonding in Crystalline Materials. New York: Cambridge University Press 2001.

(38) Ma, L.; Hao, F.; Stoumpos, C. C.; Phelan, B. T.; Wasielewski, M. R.; Kanatzidis, M. G. Carrier Diffusion Lengths of over $500 \mathrm{~nm}$ in Lead-Free Perovskite $\mathrm{CH}_{3} \mathrm{NH}_{3} \mathrm{SnI}_{3}$ Films. J. Am. Chem. Soc. 2016, 138, 14750-14755.

(39) Xie, G.; Xu, L.; Sun, L.; Xiong, Y.; Wu, P.; Hu, B. Insight into the Reaction Mechanism of Water, Oxygen and Nitrogen Molecules on a Tin Iodine Perovskite Surface. J. Mater. Chem. A 2019, 7, 5779-5793.

(40) Li, F.; Zhang, C.; Huang, J.-H.; Fan, H.; Wang, H.; Wang, P.; Zhan, C.; Liu, C.-M.; Li, X.; Yang, L.-M.; et al. A Cation-Exchange Approach for the Fabrication of Efficient Methylammonium Tin Iodide Perovskite Solar Cells. Angew. Chem. Int. Ed. 2019, 58, 6688-6692. (41) Baikie, T.; Fang, Y.; Kadro, J. M.; Schreyer, M.; Wei, F.; Mhaisalkar, S. G.; Graetzel, M.; White, T. J. Synthesis and Crystal Chemistry of the Hybrid Perovskite $\left(\mathrm{CH}_{3} \mathrm{NH}_{3}\right) \mathrm{PbI}_{3}$ for SolidState Sensitized Solar Cell Applications. J. Mater. Chem. A 2013, 1, 5628-5641.

(42) Amat, A.; Mosconi, E.; Ronca, E.; Quarti, C.; Umari, P.; Nazeeruddin, M. K.; Grätzel, M.; Angelis, F. D. Cation-Induced Band-Gap Tuning in Organohalide Perovskites: Interplay of SpinOrbit Coupling and Octahedra Tilting. Nano Lett. 2014, 14, 3608-3616.

(43) Shi, T.; Zhang, H.-S.; Meng, W.; Teng, Q.; Liu, M.; Yang, X.; Yan, Y.; Yip, H.-L.; Zhao, Y.J. Effects of Organic Cations on the Defect Physics of Tin Halide Perovskites. J. Mater. Chem. A 2017, 5, 15124-15129.

(44) Koh, T. M.; Krishnamoorthy, T.; Yantara, N.; Shi, C.; Leong, W. L.; Boix, P. P.; Grimsdale, 
A. C.; Mhaisalkar, S. G.; Mathews, N. Formamidinium Tin-Based Perovskite with Low $E_{\mathrm{g}}$ for Photovoltaic Applications. J. Mater. Chem. A 2015, 3, 14996-15000.

(45) Liao, W.; Zhao, D.; Yu, Y.; Grice, C. R.; Wang, C.; Cimaroli, A. J.; Schulz, P.; Meng, W.; Zhu, K.; Xiong, R.-G.; et al. Lead-Free Inverted Planar Formamidinium Tin Triiodide Perovskite Solar Cells Achieving Power Conversion Efficiencies up to 6.22\%. Adv. Mater. 2016, 28, 93339340.

(46) Jokar, E.; Chien, C.-H.; Fathi, A.; Rameez, M.; Chang, Y.-H.; Diau, E. W.-G. Slow Surface Passivation and Crystal Relaxation with Additives to Improve Device Performance and Durability for Tin-Based Perovskite Solar Cells. Energy Environ. Sci. 2018, 11, 2353-2362.

(47) Milot, R. L.; Eperon, G. E.; Green, T.; Snaith, H. J.; Johnston, M. B.; Herz, L. M. Radiative Monomolecular Recombination Boosts Amplified Spontaneous Emission in $\mathrm{HC}\left(\mathrm{NH}_{2}\right)_{2} \mathrm{SnI}_{3}$ Perovskite Films. J. Phys. Chem. Lett. 2016, 7, 4178-4184.

(48) Xu, X.; Chueh, C.-C.; Jing, P.; Yang, Z.; Shi, X.; Zhao, T.; Lin, L. Y.; Jen, A. K.-Y. HighPerformance near-IR Photodetector Using Low-Bandgap $\mathrm{MA}_{0.5} \mathrm{FA}_{0.5} \mathrm{~Pb}_{0.5} \mathrm{Sn}_{0.5} \mathrm{I}_{3}$ Perovskite. $A d v$. Funct. Mater. 2017, 27, 1701053.

(49) Wang, W.; Zhao, D.; Zhang, F.; Li, L.; Du, M.; Wang, C.; Yu, Y.; Huang, Q.; Zhang, M.; Li, L.; et al. Highly Sensitive Low-Bandgap Perovskite Photodetectors with Response from Ultraviolet to the near-Infrared Region. Adv. Funct. Mater. 2017, 27, 1703953.

(50) Sutton, R. J.; Eperon, G. E.; Miranda, L.; Parrott, E. S.; Kamino, B. A.; Patel, J. B.; Hörantner, M. T.; Johnston, M. B.; Abbas Haghighirad, A.; Moore, D. T.; et al. Bandgap-Tunable Cesium Lead Halide Perovskites with High Thermal Stability for Efficient Solar Cells. Adv. Energy Mater. 2016, $6,1502458$.

(51) Chung, I.; Song, J.-H.; Im, J.; Androulakis, J.; Malliakas, C. D.; Li, H.; Freeman, A. J.; Kenney, J. T.; Kanatzidis, M. G. CsSnI 3 : Semiconductor or Metal? High Electrical Conductivity and Strong near-Infrared Photoluminescence from a Single Material. High Hole Mobility and Phase-Transitions. J. Am. Chem. Soc. 2012, 134, 8579-8587.

(52) Huang, L.-y.; Lambrecht, W. R. L. Electronic Band Structure, Phonons, and Exciton Binding Energies of Halide Perovskites $\mathrm{CsSnCl}_{3}, \mathrm{CsSnBr}_{3}$, and $\mathrm{CsSnI}_{3}$. Phys. Rev. B 2013, 88, 165203.

(53) Chen, Z.; Wang, J. J.; Ren, Y.; Yu, C.; Shum, K. Schottky Solar Cells Based on CsSnI 3 Thin Films. Appl. Phys. Lett. 2012, 101, 093901.

(54) Huang, L.-Y.; Lambrecht, W. R. L. Lattice Dynamics in Perovskite Halides CsSnX 3 with X 
=I, Br, Cl. Phys. Rev. B 2014, 90, 195201.

(55) da Silva, E. L.; Skelton, J. M.; Parker, S. C.; Walsh, A. Phase Stability and Transformations in the Halide Perovskite Cssni3. Phys. Rev. B 2015, 91, 144107.

(56) Fabini, D. H.; Laurita, G.; Bechtel, J. S.; Stoumpos, C. C.; Evans, H. A.; Kontos, A. G.; Raptis, Y. S.; Falaras, P.; Ven, A. V. d.; Kanatzidis, M. G.; et al. Dynamic Stereochemical Activity of the $\mathrm{Sn}^{2+}$ Lone Pair in Perovskite CsSnBr 3. J. Am. Chem. Soc. 2016, 138, 11820-11832.

(57) Waghmare, U. V.; Spaldin, N. A.; Kandpal, H. C.; Seshadri, R. First-principles Indicators of Metallicity and Cation off-centricity in The IV-VI Rocksalt Chalcogenides of Divalent Ge, Sn, and Pb. Phys. Rev. B: Condens. Matter Mater. Phys. 2003, 67, 125111.

(58) Jung, Y.-K.; Lee, J.-H.; Walsh, A.; Soon, A. Influence of Rb/Cs Cation-Exchange on Inorganic Sn Halide Perovskites: From Chemical Structure to Physical Properties. Chem. Mater. 2017, 29, 3181-3188.

(59) Yu, Z. -L.; Ma, Q.-R.; Zhao, Y.-Q.; Liu, B.; Cai, M.-Q. Surface Termination-A Key Factor to Influence Electronic and Optical Properties of $\mathrm{CsSnI}_{3}$. J. Phys. Chem. C 2018, 122, 9275-9282. (60) Bernasconi, A.; Rizzo, A.; Andrea Listorti; Mahata, A.; Mosconi, E.; Angelis, F. D.; Malavasi, L. Synthesis, Properties, and Modeling of $\mathrm{Cs}_{1-\mathrm{X}} \mathrm{Rb}_{\mathrm{X}} \mathrm{SnBr}_{3}$ Solid Solution: A New Mixed-Cation Lead-Free All-Inorganic Perovskite System. Chem. Mater. 2019, 31, 3527-3533.

(61) Li, B.; Long, R.; Xia, Y.; Mi, Q. All-Inorganic Perovskite $\mathrm{CsSnBr}_{3}$ as a Thermally Stable, Free-Carrier Semiconductor. Angew. Chem. In t. Ed. 2018, 57, 13154-13158.

(62) Wu, B.; Zhou, Y.; Xing, G.; Xu, Q.; Garces, H. F.; Solanki, A.; Goh, T. W.; Padture, N. P.; Sum, T. C. Long Minority-Carrier Diffusion Length and Low Surface-Recombination Velocity in Inorganic Lead-Free $\mathrm{CsSnI}_{3}$ Perovskite Crystal for Solar Cells. Adv. Funct. Mater. 2017, 27 , 1604818.

(63) Chung, I.; Lee, B.; He, J.; Chang, R. P. H.; Kanatzidis, M. G. All-Solid-State Dye-Sensitized Solar Cells with High Efficiency. Nature 2012, 485, 486-489.

(64) Chen, M.; Ju, M.-G.; Garces, H. F.; Carl, A. D.; Ono, L. K.; Hawash, Z.; Zhang, Y.; Shen, T.; Qi, Y.; Grimm, R. L.; et al. Highly Stable and Efficient All-Inorganic Lead-Free Perovskite Solar Cells with Native-Oxide Passivation. Nat. Commun. 2019, 10, 16.

(65) Abrahams, I.; Demetriou, D. Z. Inert Pair Effects in Tin and Lead Dihalides Crystal Structure of Tin(II) Bromide. J. Solid State Chem. 2000, 149, 28-32.

(66) Travis, W.; Glover, E. N. K.; Bronstein, H.; Scanlon, D. O.; Palgrave, R. G. On the 
Application of the Tolerance Factor to Inorganic and Hybrid Halide Perovskites: A Revised System. Chem. Sci. 2016, 7, 4548-4556.

(67) Dang, Y.; Zhong, C.; Zhang, G.; Ju, D.; Wang, L.; Xia, S.; Xia, H.; Tao, X. Crystallographic Investigations into Properties of Acentric Hybrid Perovskite Single Crystals $\mathrm{NH}\left(\mathrm{CH}_{3}\right)_{3} \mathrm{SnX}_{3}(\mathrm{X}=$ Cl, Br). Chem. Mater. 2016, 28, 6968-6974.

(68) Tsarev, S.; Boldyreva, A. G.; Luchkin, S. Y.; Elshobaki, M.; Afanasov, M. I.; Stevensona, K. J.; Troshin, P. A. Hydrazinium-Assisted Stabilisation of Methylammonium Tin Iodide for LeadFree Perovskite Solar Cells. J. Mater. Chem. A 2018, 6, 21389-21395.

(69) Ishibashi, H.; Katayama, M.; Tanaka, S.; Kaji, T. Hybrid Perovskite Solar Cells Fabricated from Guanidine Hydroiodide and Tin Iodide. Sci. Rep. 2017, 7, 4969.

(70) Stoumpos, C. C.; Mao, L.; Malliakas, C. D.; Kanatzidis, M. G. Structure-Band Gap Relationships in Hexagonal Polytypes and Low-Dimensional Structures of Hybrid Tin Iodide Perovskites. Inorg. Chem. 2017, 56, 56-73.

(71) Ke, W.; Stoumpos, C. C.; Spanopoulos, I.; Mao, L.; Chen, M.; Wasielewski, M. R.; Kanatzidis, M. G. Efficient Lead-Free Solar Cells Based on Hollow \{en\} MASnI 3 Perovskites. J. Am. Chem. Soc. 2017, 139, 14800-14806.

(72) Ke, W.; Stoumpos, C. C.; Zhu, M.; Mao, L.; Spanopoulos, I.; Jian Liu, O. Y. K.; Chen, M.; Sarma, D.; Zhang, Y.; Wasielewski, M. R.; et al. Enhanced Photovoltaic Performance and Stability with a New Type of Hollow 3D Perovskite \{en\}FASnI $3 . S c i . A d v .2017$, , e1701293.

(73) Ke, W.; Stoumpos, C. C.; Spanopoulos, I.; Chen, M.; Wasielewski, M. R.; Kanatzidis, M. G. Diammonium Cations in the $\mathrm{FASnI}_{3}$ Perovskite Structure Lead to Lower Dark Currents and More Efficient Solar Cells. ACS Energy Lett. 2018, 3, 1470-1476.

(74) Tsai, C.-M.; Lin, Y.-P.; Pola, M. K.; Narra, S.; Jokar, E.; Yang, Y.-W.; Diau, E. W.-G. Control of Crystal Structures and Optical Properties with Hybrid Formamidinium and 2Hydroxyethylammonium Cations for Mesoscopic Carbon-Electrode Tin-Based Perovskite Solar Cells. ACS Energy Lett. 2018, 3, 2077-2085.

(75) Smith, I. C.; Hoke, E. T.; Solis-Ibarra, D.; McGehee, M. D.; Karunadasa, H. I. A Layered Hybrid Perovskite Solar-Cell Absorber with Enhanced Moisture Stability. Angew. Chem. Int. Ed. 2014, 53, 11232-11235.

(76) Cao, D. H.; Stoumpos, C. C.; Farha, O. K.; Hupp, J. T.; Kanatzidis, M. G. 2D Homologous Perovskites as Light-Absorbing Materials for Solar Cell Applications. J. Am. Chem. Soc. 2015, 
137, 7843-7850.

(77) Lin, H. R.; Zhou, C. K.; Tian, Y.; Siegrist, T.; Ma, B. W. Low-Dimensional Organometal Halide Perovskites. ACS Energy Lett. 2018, 3, 54-62.

(78) Pan, J.; Quan, L.; Zhao, Y.; Peng, W.; Murali, B.; Sarmah, S. P.; Yuan, M.; Sinatra, L.; Alyami, N. M.; Liu, J.; et al. Highly Efficient Perovskite-Quantum-Dot Light-Emitting Diodes by Surface Engineering. Adv. Mater. 2016, 28, 8718-8725.

(79) Qiu, J.; Xia, Y.; Chen, Y.; Huang, W. Management of Crystallization Kinetics for Efficient and Stable Low-Dimensional Ruddlesden-Popper (LDRP) Lead-Free Perovskite Solar Cells. Adv. Sci. 2019, 6, 1800793.

(80) Wang, A.; Guo, Y.; Zhou, Z.; Niu, X.; Wang, Y.; Muhammad, F.; Li, H.; Zhang, T.; Wang, J.; Nie, S.; et al. Aqueous Acid-Based Synthesis of Lead-Free Tin Halide Perovskites with nearUnity Photoluminescence Quantum Efficiency. Chem. Sci. 2019, 10, 4573-4579.

(81) Shen, H.; Li, J.; Wang, H.; Ma, J.; Wang, J.; Luo, H.; Li, D., Two-Dimensional Lead-Free Perovskite $\left(\mathrm{C}_{6} \mathrm{H}_{5} \mathrm{C}_{2} \mathrm{H}_{4} \mathrm{NH}_{3}\right)_{2} \mathrm{CsSn}_{2} \mathrm{I}_{7}$ with High Hole Mobility. J. Phys. Chem. Lett. 2019, 10, 7-12. (82) Wang, L.; Chen, P.; Thongprong, N.; Young, M.; Kuttipillai, P. S.; Jiang, C.; Zhang, P.; Sun, K.; Duxbury, P. M.; Lunt, R. R. Unlocking the Single-Domain Epitaxy of Halide Perovskites. Adv. Mater. Interfaces 2017, 4, 1701003.

(83) Wong, A. B.; Bekenstein, Y.; Kang, J.; Kley, C. S.; Kim, D.; Gibson, N. A.; Zhang, D.; Yu, Y.; Leone, S. R.; Wang, L.-W.; et al. Strongly Quantum Confined Colloidal Cesium Tin Iodide Perovskite Nanoplates: Lessons for Reducing Defect Density and Improving Stability. Nano Lett. 2018, 18, 2060-2066.

(84) Zhang, X.; Wang, C.; Zhang, Y.; Zhang, X.; Wang, S.; Lu, M.; Cui, H.; Kershaw, S. V.; Yu, W. W.; Rogach, A. L. Bright Orange Electroluminescence from Lead-Free Two-Dimensional Perovskites. ACS Energy Lett. 2019, 4, 242-248.

(85) Wang, A.; Guo, Y.; Muhammad, F.; Deng, Z. Controlled Synthesis of Lead-Free Cesium Tin Halide Perovskite Cubic Nanocages with High Stability. Chem. Mater. 2017, 29, 6493-6501.

(86) Jellicoe, T. C.; Richter, J. M.; Glass, H. F. J.; Tabachnyk, M.; Brady, R.; Dutton, S. E.; Rao, A.; Friend, R. H.; Credgington, D.; Greenham, N. C.; et al. Synthesis and Optical Properties of Lead-Free Cesium Tin Halide Perovskite Nanocrystals. J. Am. Chem. Soc. 2016, 138, 2941-2944. (87) Zhou, C.; Lin, H.; Shi, H.; Tian, Y.; Pak, C.; Shatruk, M.; Zhou, Y.; Djurovich, P.; Du, M.H.; Ma, B. A Zero-Dimensional Organic Seesaw-Shaped Tin Bromide with Highly Efficient 
Strongly Stokes-Shifted Deep-Red Emission. Angew. Chem. Int. Ed. 2018, 57, 1021-1024.

(88) Wang, F.; Jiang, X.; Chen, H.; Shang, Y.; Liu, H.; Wei, J.; Zhou, W.; He, H.; Liu, W.; Ning, Z. 2D-Quasi-2D-3D Hierarchy Structure for Tin Perovskite Solar Cells with Enhanced Efficiency and Stability. Joule 2018, 2, 2732-2743.

(89) Wang, Y.; Tu, J.; Li, T.; Tao, C.; Deng, X.; Li, Z. Convenient Preparation of CsSnI 3 Quantum Dots, Excellent Stability, and the Highest Performance of Lead-Free Inorganic Perovskite Solar Cells So Far. J. Mater. Chem. A 2019, 7, 7683-7690.

(90) Hu, T.; Smith, M. D.; Dohner, E. R.; Sher, M.-J.; Wu, X.; Trinh, M. T.; Fisher, A; Corbett, J.; Zhu, X.-Y.; Karunadasa, H. I., et al. Mechanism for Broadband White-Light Emission from Two-Dimensional (110) Hybrid Perovskites. J. Phys. Chem. Lett. 2016, 7, 2258-2263.

(91) Dohner, E. R.; Hoke, E. T.; Karunadasa, H. I. Self-Assembly of Broadband White-Light Emitters. J. Am. Chem. Soc. 2014, 136, 1718-1721.

(92) Kabanov, V. V.; Mashtakov, O. Y. Electron Localization with and without Barrier Formation. Phys. Rev. B: Condens. Matter Mater. Phys. 1993, 47, 6060-6064.

(93) Zhou, C.; Tian, Y.; Wang, M.; Rose, A.; Besara, T.; Doyle, N. K.; Yuan, Z.; Wang, J. C.; Clark, R.; Hu, Y.; et al. Low-Dimensional Organic Tin Bromide Perovskites and Their Photoinduced Structural Transformation. Angew. Chem. Int. Ed. 2017, 56, 9018-9022.

(94) Mao, L.; Tsai, H.; Nie, W.; Ma, L.; Im, J.; Stoumpos, C. C.; Malliakas, C. D.; Hao, F.; Wasielewski, M. R.; Mohite, A. D.; et al. Role of Organic Counterion in Lead-and Tin-Based TwoDimensional Semiconducting Iodide Perovskites and Application in Planar Solar Cells. Chem. Mater. 2016, 28, 7781-7792.

(95) Tao, S.; Schmidt, I.; Brocks, G.; Jiang, J.; Tranca, I.; Meerholz, K.; Olthof, S. Absolute Energy Level Positions in Tinand Lead-Based Halide Perovskites. Nat. Commun. 2019, 10, 1-10.

(96) Hao, F.; Stoumpos, C. C.; Guo, P.; Zhou, N.; Marks, T. J.; Chang, R. P. H.; Kanatzidis, M. G. Solvent-Mediated Crystallization of $\mathrm{CH}_{3} \mathrm{NH}_{3} \mathrm{SnI}_{3}$ Films for Heterojunction Depleted Perovskite Solar Cells. J. Am. Chem. Soc. 2015, 137, 11445-11452.

(97) Hao, F.; Stoumpos, C. C.; Chang, R. P. H.; Kanatzidis, M. G. Anomalous Band Gap Behavior in Mixed Sn and $\mathrm{Pb}$ Perovskites Enables Broadening of Absorption Spectrum in Solar Cells. $J$. Am. Chem. Soc. 2014, 136, 8094-8099.

(98) Zhao, B.; Abdi-Jalebi, M.; Tabachnyk, M.; Glass, H.; Kamboj, V. S.; Nie, W. A.; Pearson, J.; Puttisong, Y.; Gödel, K. C.; Beere, H. E.; et al. High Open-Circuit Voltages in Tin-Rich Low- 
Bandgap Perovskite-Based Planar Heterojunction Photovoltaics. Adv. Mater. 2017, 29, 1604744. (99) Im, J.; Stoumpos, C. C.; Jin, H.; Freeman, A. J.; Kanatzidis, M. G. Antagonism between SpinOrbit Coupling and Steric Effects Causes Anomalous Band Gap Evolution in the Perovskite Photovoltaic Materials $\mathrm{CH}_{3} \mathrm{NH}_{3} \mathrm{Sn}_{1-\mathrm{x}} \mathrm{Pb}_{\mathrm{x}} \mathrm{I}_{3}$. J. Phys. Chem. Lett. 2015, 6, 3503-3509.

(100) Goyal, A.; McKechnie, S.; Pashov, D.; Tumas, W.; Schilfgaarde, M. v.; Stevanović, V. Origin of Pronounced Nonlinear Band Gap Behavior in Lead-Tin Hybrid Perovskite Alloys. Chem. Mater. 2018, 30, 3920-3928.

(101) Pisanu, A.; Mahata, A.; Mosconi, E.; Patrini, M.; Quadrelli, P.; Milanese, C.; Angelis, F. D.; Malavasi, L. Exploring the Limits of Three-Dimensional Perovskites: The Case of $\mathrm{FAPb}_{1-\mathrm{x}} \mathrm{Sn}_{\mathrm{x}} \mathrm{Br}_{3}$. ACS Energy Lett. 2018, 3, 1353-1359.

(102) Parrott, E. S.; Green, T.; Milot, R. L.; Johnston, M. B.; Snaith, H. J.; Herz, L. M. Interplay of Structural and Optoelectronic Properties in Formamidinium Mixed Tin-Lead Triiodide Perovskites. Adv. Funct. Mater. 2018, 28, 1802803.

(103) Nagane, S.; Ghosh, D.; Hoye, R. L. Z.; Zhao, B.; Ahmad, S.; Walker, A. B.; Islam, M. S.; Ogale, S.; Sadhanala, A. Lead-Free Perovskite Semiconductors Based on Germanium-Tin Solid Solutions: Structural and Optoelectronic Properties. J. Phys. Chem. C 2018, 122, 5940-5947.

(104) Lee, S.; Levi, R. D.; Qu, W.; Lee, S. C.; Randall, C. A. Band-gap nonlinearity in perovskite structured solid solutions. J. Appl. Phys. 2010, 107, 023523.

(105) Yamini, S. A.; Patterson, V.; Santos, R. Band-Gap Nonlinearity in Lead Chalcogenide (PbQ, $\mathrm{Q}=\mathrm{Te}$, Se, S) Alloys. ACS Omega 2017, 2, 3417-3423.

(106) Ferrara, C.; Patrini, M.; Pisanu, A.; Quadrelli, P.; Milanese, C.; Tealdi, C.; Malavasi, L. Wide Band-Gap Tuning in Sn-Based Hybrid Perovskites through Cation Replacement: The $\mathrm{FA}_{1-\mathrm{x}} \mathrm{MA}_{\mathrm{x}} \mathrm{SnBr}_{3}$ Mixed System. J. Mater. Chem. A 2017, 5, 9391-9395.

(107) Lü, X.; Wang, Y.; Stoumpos, C. C.; Hu, Q.; Guo, X.; Chen, H.; Yang, L.; Smith, J. S.; Yang, W.; Zhao, Y.; et al. Enhanced Structural Stability and Photo Responsiveness of $\mathrm{CH}_{3} \mathrm{NH}_{3} \mathrm{SnI}_{3}$ Perovskite Via Pressure-Induced Amorphization and Recrystallization. Adv. Mater. 2016, 28, 8663-8668.

(108) Wang, L.; Ou, T.; Wang, K.; Xiao, G.; Gao, C.; Zou, B. Pressure-Induced Structural Evolution, Optical and Electronic Transitions of Nontoxic Organometal Halide Perovskite-Based Methylammonium Tin Chloride. Appl. Phys. Lett. 2017, 111, 233901.

(109) Shi, Y.; Ma, Z.; Zhao, D.; Chen, Y.; Cao, Y.; Wang, K.; Xiao, G.; Zou, B. Pressure-Induced 
Emission (PIE) of One-Dimensional Organic Tin Bromide Perovskites. J. Am. Chem. Soc. 2019, 141, 6504-6508.

(110) Wang, J.; Shen, H.; Li, W.; Wang, S.; Li, J.; Li, D. The Role of Chloride Incorporation in Lead-Free 2D Perovskite $(B A)_{2} \mathrm{SnI}_{4}$ : Morphology, Photoluminescence, Phase Transition, and Charge Transport. Adv. Sci. 2019, 6, 1802019.

(111) Cheng, P.; Wu, T.; Liu, J.; Deng, W.-Q.; Han, K. Lead-Free, Two-Dimensional Mixed Germanium and Tin Perovskites. J. Phys. Chem. Lett. 2018, 9, 2518-2522.

(112) Stoumpos, C. C.; Malliakas, C. D.; Kanatzidis, M. G. Semiconducting Tin and Lead Iodide Perovskites with Organic Cations: Phase Transitions, High Mobilities, and Near-Infrared Photoluminescent Properties. Inorg. Chem. 2013, 52, 9019-9038.

(113) Herz, L. M. Charge-Carrier Mobilities in Metal Halide Perovskites: Fundamental Mechanisms and Limits. ACS Energy Lett. 2017, 2, 1539-1548.

(114) Parrott, E. S.; Milot, R. L.; Stergiopoulos, T.; Snaith, H. J.; Johnston, M. B.; Herz, L. M. Effect of Structural Phase Transition on Charge-Carrier Lifetimes and Defects in $\mathrm{CH}_{3} \mathrm{NH}_{3} \mathrm{SnI}_{3}$ Perovskite. J. Phys. Chem. Lett. 2016, 7, 1321-1326.

(115) Milot, R. L.; Klug, M. T.; Davies, C. L.; Wang, Z.; Kraus, H.; Snaith, H. J.; Johnston, M. B.; Herz, L. M. The Effects of Doping Density and Temperature on the Optoelectronic Properties of Formamidinium Tin Triiodide Thin Films. Adv. Mater. 2018, 30, 1804506.

(116) Gupta, S.; Bendikov, T.; Hodes, G.; Cahen, D., CsSnBr 3 A Lead-Free Halide Perovskite for Long-Term Solar Cell Application: Insights on $\mathrm{SnF}_{2}$ Addition. ACS Energy Lett. 2016, 1, 10281033.

(117) Ng, C. H.; Nishimura, K.; Ito, N.; Hamada, K.; Hirotani, D.; Wang, Z.; Yang, F.; likubo, S.; Shen, Q.; Yoshino, K.; et al. Role of $\mathrm{GeI}_{2}$ and $\mathrm{SnF}_{2}$ Additives for SnGe Perovskite Solar Cells. Nano Energy 2019, 58, 130-137.

(118) Feng, H.-J.; Paudel, T. R.; Tsymbal, E. Y.; Zeng, X. C. Tunable Optical Properties and Charge Separation in $\mathrm{CH}_{3} \mathrm{NH}_{3} \mathrm{Sn}_{\mathrm{x}} \mathrm{Pb}_{1-\mathrm{x}} \mathrm{I}_{3} / \mathrm{TiO}_{2}$-Based Planar Perovskites Cells. J. Am. Chem. Soc. 2015, 137, 8227-8236.

(119) Shen, Q.; Ogomi, Y.; Chang, J.; Toyoda, T.; Fujiwara, K.; Yoshino, K.; Sato, K.; Yamazaki, K.; Akimoto, M.; Kuga, Y.; et al. Optical Absorption, Charge Separation and Recombination Dynamics in $\mathrm{Sn} / \mathrm{Pb}$ Cocktail Perovskite Solar Cells and Their Relationships to Photovoltaic Performances. J. Mater. Chem. A 2015, 3, 9308-9316. 
(120) Fu, J.; Xu, Q.; Han, G.; Wu, B.; Huan, C. H. A.; Leek, M. L.; Sum, T. C. Hot Carrier Cooling Mechanisms in Halide Perovskites. Nat. Commun. 2017, 8, 1300.

(121) Li, M.; Bhaumik, S.; Goh, T. W.; Kumar, M. S.; Yantara, N.; Grätzel, M.; Mhaisalkar, S.; Mathews, N.; Sum, T. C. Slow Cooling and Highly Efficient Extraction of Hot Carriers in Colloidal Perovskite Nanocrystals. Nat. Commun. 2017, 8, 14350.

(122) Fang, H.-H.; Adjokatse, S.; Shao, S.; Even, J.; Loi, M. A. Long-Lived Hot-Carrier Light Emission and Large Blue Shift in Formamidinium Tin Triiodide Perovskites. Nat. Commun. 2018, 9, 243.

(123) Verma, S. D.; Gu, Q.; Sadhanala, A.; Venugopalan, V.; Rao, A. Slow Carrier Cooling in Hybrid Pb-Sn Halide Perovskites. ACS Energy Lett. 2019, 4, 736-740.

(124) Herz, L. M. How Lattice Dynamics Moderate the Electronic Properties of Metal-Halide Perovskites. J. Phys. Chem. Lett. 2018, 23, 6853-6863.

(125) Zhang, J.; Wu, T.; Duan, J.; Ahmadi, M.; Jiang, F.; Zhou, Y.; Hu, B. Exploring Spin-Orbital Coupling Effects on Photovoltaic Actions in Sn and Pb Based Perovskite Solar Cells. Nano Energy 2017, 38, 297-303.

(126) Ahmadi, M.; Collins, L.; Puretzky, A.; Zhang, J.; Keum, J. K.; Lu, W.; Ivanov, I.; Kalinin, S. V.; Hu, B. Exploring Anomalous Polarization Dynamics in Organometallic Halide Perovskites. Adv. Mater. 2018, 30, 1705298.

(127) Liang, H.; Yuan, F.; Johnston, A.; Gao, C.; Choubisa, H.; Gao, Y.; Wang, Y. K.; Sagar, L. K.; Sun, B.; Li, P.; et al. High Color Purity Lead-Free Perovskite Light-Emitting Diodes via Sn Stabilization. Adv. Sci. 2020, 7, 1903213.

(128) Li, T.; Chen, X.; Wang, X.; Lu, H.; Yan, Y.; Beard, M. C.; Mitzi, D. B. Origin of BroadBand Emission and Impact of Structural Dimensionality in Tin-Alloyed Ruddlesden-Popper Hybrid Lead Iodide Perovskites. ACS Energy Lett. 2020, 5, 347-352.

(129) Ushakova, E. V.; Cherevkov, S. A.; Kuznetsova, V. A.; Baranov, A. V. Lead-Free Perovskites for Lighting and Lasing Applications: A Minireview. Materials (Basel). 2019, 12, 3845.

(130) Frost, J. M.; Butler, K. T.; Brivio, F.; Hendon, C. H.; Van Schilfgaarde, M.; Walsh, A., Atomistic Origins of High-Performance in Hybrid Halide Perovskite Solar Cells. Nano Lett. 2014, 14, 2584-2590.

(131) Tong, C. J.; Geng, W.; Tang, Z. K.; Yam, C. Y.; Fan, X. L.; Liu, J.; Lau, W. M.; Liu, L. M. 
Uncovering the Veil of the Degradation in Perovskite $\mathrm{CH}_{3} \mathrm{NH}_{3} \mathrm{PbI}_{3}$ upon Humidity Exposure: A First-Principles Study. J. Phys. Chem. Lett. 2015, 6, 3289-3295.

(132) Mosconi, E.; Azpiroz, J. M.; De Angelis, F., Ab Initio Molecular Dynamics Simulations of Methylammonium Lead Iodide Perovskite Degradation by Water. Chem. Mater. 2015, 27, 4885-4892.

(133) Aristidou, N.; Sanchez-Molina, I.; Chotchuangchutchaval, T.; Brown, M.; Martinez, L.; Rath, T.; Haque, S. A., The Role of Oxygen in the Degradation of Methylammonium Lead Trihalide Perovskite Photoactive Layers. Angew. Chem., Int. Ed. 2015, 54, 8208-8212.

(134) Marshall, K. P.; M. Walker; I. Walton, R.; Hatton, R. A. Enhanced Stability and Efficiency in Hole-Transport-Layer-Free $\mathrm{CsSnI}_{3}$ Perovskite Photovoltaics. Nat. Energy 2016, 1, 178.

(135) Heo, J. H.; Kim, J.; Kim, H.; Moon, S. H.; Im, S. H.; Hong, K.-H. Roles of SnX2 (X = F, Cl, Br) Additives in Tin-Based Halide Perovskites toward Highly Efficient and Stable Lead-Free Perovskite Solar Cells. J. Phys. Chem. Lett. 2018, 9, 6024-6031.

(136) Song, T.-B.; Yokoyama, T.; Aramaki, S.; Kanatzidis, M. G. Performance Enhancement of Lead-Free Tin-Based Perovskite Solar Cells with Reducing Atmosphere-Assisted Dispersible Additive. ACS Energy Lett. 2017, 2, 897-903.

(137) Lee, Y. M.; Park, J.; Yu, B. D.; Hong, S.; Jung, M.-C.; Nakamura, M. Surface Instability of Sn-Based Hybrid Perovskite Thin Film, $\mathrm{CH}_{3} \mathrm{NH}_{3} \mathrm{SnI}_{3}$ : The Origin of Its Material Instability. $J$. Phys. Chem. Lett. 2018, 9, 2293-2297.

(138) Leijtens, T.; Prasanna, R.; Gold-Parker, A.; Toney, M. F.; McGehee, M. D. Mechanism of Tin Oxidation and Stabilization by Lead Substitution in Tin Halide Perovskites. ACS Energy Lett. 2017, 2, 2159-2165.

(139) Kubicki, D. J.; Prochowicz, D.; Salager, E.; Rakhmatullin, A.; Grey, C. P.; Emsley, L.; Stranks, S. D. Local Structure and Dynamics in Methylammonium, Formamidinium, and Cesium Tin(II) Mixed-Halide Perovskites from ${ }^{119}$ Sn Solid-State NMR. J. Am. Chem. Soc. 2020, 142, 7813-7826.

(140) Nishikubo, R.; Ishida, N.; Katsuki, Y.; Wakamiya, A.; Saeki, A. Minute-Scale Degradation and Shift of Valence-Band Maxima of $\left(\mathrm{CH}_{3} \mathrm{NH}_{3}\right) \mathrm{SnI}_{3}$ and $\mathrm{HC}\left(\mathrm{NH}_{2}\right)_{2} \mathrm{SnI}_{3}$ Perovskites Upon Air Exposure. J. Phys. Chem. C 2017, 121, 19650-19656.

(141) Marshall, K. P.; Walker, M.; Walton, R. I.; Hatton, R. A. Elucidating the Role of the HoleExtracting Electrode on the Stability and Efficiency of Inverted $\mathrm{CsSnI}_{3} / \mathrm{C}_{60}$ Perovskite 
Photovoltaics. J. Mater. Chem. A 2017, 5, 21836-21845.

(142) Kumar, M. H.; Dharani, S.; Leong, W. L.; Boix, P. P.; Prabhakar, R. R.; Baikie, T.; Shi, C.; Ding, H.; Ramesh, R.; Asta, M.; et al. Lead-Free Halide Perovskite Solar Cells with High Photocurrents Realized through Vacancy Modulation. Adv. Mater. 2014, 26, 7122-7127.

(143) Song, T.-B.; Yokoyama, T.; Logsdon, J.; Wasielewski, M. R.; Aramaki, S.; Kanatzidis, M. G., Piperazine Suppresses Self-Doping in $\mathrm{CsSnI}_{3}$ Perovskite Solar Cells. ACS Appl. Energy Mater. 2018, 1, 4221-4226.

(144) Marshall, K. P.; Walker, M.; Walton, R. I.; Hatton, R. A. Enhanced Stability and Efficiency in Hole-Transport-Layer-Free $\mathrm{CsSnI}_{3}$ Perovskite Photovoltaics. Nat. Energy 2016, 1, 16178. (145) Yu, Y.; Zhao, D.; Grice, C. R.; Meng, W.; Wang, C.; Liao, W.; Cimaroli, A. J.; Zhang, H.; Zhu, K.; Yan, Y. Thermally Evaporated Methylammonium Tin Triiodide Thin Films for LeadFree Perovskite Solar Cell Fabrication. RSC Adv. 2016, 6, 90248-90254.

(146) Eperon, G. E.; Ginger, D. S. B-Site Metal Cation Exchange in Halide Perovskites. ACS Energy Lett. 2017, 2, 1190-1196.

(147) Lee, S. J.; Shin, S. S.; Kim, Y. C.; Kim, D.; Ahn, T. K.; Noh, J. H.; Seo, J.; Seok, S. I. Fabrication of Efficient Formamidinium Tin Iodide Perovskite Solar Cells through $\mathrm{SnF}_{2}-\mathrm{Pyrazine}$ Complex. J. Am. Chem. Soc. 2016, 138, 3974-3977.

(148) Zhu, Z.; Chueh, C.-C.; Li, N.; Mao, C.; Jen, A. K.-Y. Realizing Efficient Lead-Free Formamidinium Tin Triiodide Perovskite Solar Cells Via a Sequential Deposition Route. Adv. Mater. 2018, 30, 1703800.

(149) Zong, Y.; Zhou, Z.; Padture, M. C. N. P.; Zhou, Y. Lewis-Adduct Mediated Grain-Boundary Functionalization for Efficient Ideal-Bandgap Perovskite Solar Cells with Superior Stability. Adv. Energy Mater. 2018, 8, 1800997.

(150) Tai, Q.; Guo, X.; Tang, G.; You, P.; Ng, T.-W.; Shen, D.; Cao, J.; Liu, C.-K.; Wang, N.; Zhu, Y.; et al. Antioxidant Grain Passivation for Air-Stable Tin-Based Perovskite Solar Cells. Angew. Chem. Int. Ed. 2019, 58, $806-810$.

(151) Kim, H.; Lee, Y. H.; Lyu, T.; Yoo, J. H.; Park, T.; Oh, J. H. Boosting the Performance and Stability of Quasi Two-Dimensional Tin-Based Perovskite Solar Cells Using the Formamidinium Thiocyanate Additive. J. Mater. Chem. A 2018, 6, 18173-18182.

(152) Lian, X.; Chen, J.; Zhang, Y.; Qin, M.; Li, J.; Tian, S.; Yang, W.; Lu, X.; Wu, G.; Chen, H. Highly Efficient Sn/Pb Binary Perovskite Solar Cell Via Precursor Engineering: A Two-Step 
Fabrication Process. Adv. Funct. Mater. 2019, 29, 1807024.

(153) Ruggeri, E.; Anaya, M.; Gałkowski, K.; Delport, G.; Kosasih, F. U.; Abfalterer, A.; Mackowski, S.; Ducati, C.; Stranks, S. D. Controlling the Growth Kinetics and Optoelectronic Properties of 2D/3D Lead-Tin Perovskite Heterojunctions. Adv. Mater. 2019, 31, 1905247.

(154) Kamarudin, M. A.; Hirotani, D.; Wang, Z.; Hamada, K.; Nishimura, K.; Shen, Q.; Toyoda, T.; Iikubo, S.; Minemoto, T.; Yoshino, K.; et al. Suppression of Charge Carrier Recombination in Lead-Free Tin Halide Perovskite Via Lewis Base Post-Treatment. J. Phys. Chem. Lett. 2019, 10, 5277-5283.

(155) Liu, G.; Liu, C.; Lin, Z.; Yang, J.; Huang, Z.; Tan, L.; Chen, Y. Regulated Crystallization of Efficient and Stable Tin-Based Perovskite Solar Cells via a Self-Sealing Polymer. ACS Appl. Mater. Interfaces 2020, 12, 14049-14056.

(156) Li, C.; Ma, R.; He, X.; Yang, T.; Zhou, Z.; Yang, S.; Liang, Y.; Sun, X. W.; Wang, J.; Yan, Y.; et al. In Situ Tin(II) Complex Antisolvent Process Featuring Simultaneous Quasi-Core-Shell Structure and Heterojunction for Improving Efficiency and Stability of Low-Bandgap Perovskite Solar Cells. Adv. Energy Mater. 2020, 10, 1903013.

(157) He, X.; Wu, T.; Liu, X.; Wang, Y.; Meng, X.; Wu, J.; Noda, T.; Yang, X.; Moritomo, Y.; Segawae H.; et al. Highly Efficient Tin Perovskite Solar Cells Achieved in A Wide Oxygen Concentration Range. J. Mater. Chem. A, 2020, 8, 2760-2768.

(158) Song, T.-B.; Yokoyama, T.; Stoumpos, C. C.; Logsdon, J.; Cao, D. H.; Wasielewski, M. R.; Aramaki, S.; Kanatzidis, M. G. Importance of Reducing Vapor Atmosphere in the Fabrication of Tin-Based Perovskite Solar Cells. J. Am. Chem. Soc. 2017, 139, 836-842.

(159) Zhao, J.; Wei, L.; Jia, C.; Tang, H.; Su, X.; Ou, Y.; Liu, Z.; Wang, C.; Zhao, X.; Jin, H.; et al. Metallic Tin Substitution of Organic Lead Perovskite Films for Efficient Solar Cells. J. Mater. Chem. A 2018, 6, 20224-20232.

(160) Zhu, Z.; Li, N.; Zhao, D.; Wang, L.; Jen, A. K.-Y. Improved Efficiency and Stability of $\mathrm{Pb} / \mathrm{Sn}$ Binary Perovskite Solar Cells Fabricated by Galvanic Displacement Reaction. Adv. Energy Mater. 2019, 9, 1802774.

(161) Cao, J.; Tai, Q.; You, P.; Tang, G.; Wang, T.; Wang, N.; Yan, F. Enhanced Performance of Tin-Based Perovskite Solar Cells Induced by an Ammonium Hypophosphite Additive. J. Mater. Chem. A 2019, 7, 26580-26585.

(162) Zhang, T.; Li, H.; Ban, H.; Sun, Q.; Shen Y.; Wang, M. Efficient CsSnI 3 -Based Inorganic 
Perovskite Solar Cells Based on A Mesoscopic Metal Oxide Framework via Incorporating A Donor Element. J. Mater. Chem. A 2020, 8, 4118-4124.

(163) Lin, R.; Xiao, K.; Qin, Z.; Han, Q.; Zhang, C.; Wei, M.; Saidaminov, M. I.; Gao, Y.; Xu, J.; Xiao, M.; et al. Monolithic All-Perovskite Tandem Solar Cells with 24.8\% Efficiency Exploiting Comproportionation To Suppress Sn(Ii) Oxidation in Precursor Ink. Nature Energy 2019, 4, 864873.

(164) Xu, X.; Chueh, C.-C.; Yang, Z.; Rajagopal, A.; Xu, J.; Jo, S. B.; Jen, A. K.-Y. Ascorbic Acid as an Effective Antioxidant Additive to Enhance the Efficiency and Stability of $\mathrm{Pb} / \mathrm{Sn}-\mathrm{Based}$ Binary Perovskite Solar Cells. Nano Energy 2017, 34, 392-398.

(165) Kayesh, M. E.; Chowdhury, T. H.; Matsuishi, K.; Kaneko, R.; Kazaoui, S.; Lee, J.-J.; Noda, T.; Islam, A. Enhanced Photovoltaic Performance of $\mathrm{FaSnI}_{3}-$ Based Perovskite Solar Cells with Hydrazinium Chloride Coadditive. ACS Energy Lett. 2018, 3, 1584-1589.

(166) Liu, C.; Li, W.; Li, H.; Zhang, C.; Fan, J.; Mai, Y. C 60 Additive-Assisted Crystallization in $\mathrm{CH}_{3} \mathrm{NH}_{3} \mathrm{~Pb}_{0.75} \mathrm{Sn}_{0.25} \mathrm{I}_{3}$ Perovskite Solar Cells with High Stability and Efficiency. Nanoscale 2017, 9, 13967-13975.

(167) Kayesh, M. E.; Matsuishi, K.; Kaneko, R.; Kazaoui, S.; Lee, J.-J.; Noda, T.; Islam, A. Coadditive Engineering with 5-Ammonium Valeric Acid Iodide for Efficient and Stable Sn Perovskite Solar Cells. ACS Energy Lett. 2019, 4, 278-284.

(168) Meng, X.; Lin, J.; Liu, X.; He, X.; Wang, Y.; Noda, T.; Wu, T.; Yang, X.; Han, L. Highly Stable and Efficient $\mathrm{FASnI}_{3}$-Based Perovskite Solar Cells by Introducing Hydrogen Bonding. Adv. Mater. 2019, 31, 1903721.

(169) Zhang, M.; Lyu, M.; Yun, J.-H.; Noori, M.; Zhou, X.; Cooling, N. A.; Wang, Q.; Yu, H.; Dastoor, P. C.; Wang, L., Low-Temperature Processed Solar Cells with Formamidinium Tin Halide Perovskite/Fullerene Heterojunctions. Nano Res. 2016, 9, 1570-1577.

(170) Liu, C.; Fan, J.; Li, H.; Zhang, C.; Mai, Y. Highly Efficient Perovskite Solar Cells with Substantial Reduction of Lead Content. Sci. Rep. 2016, 6, 35705.

(171) Zhu, H. L.; Xiao, J.; Mao, J.; Zhang, H.; Zhao, Y.; Choy, W. C. H. Controllable Crystallization of $\mathrm{CH}_{3} \mathrm{NH}_{3} \mathrm{Sn}_{0.25} \mathrm{~Pb}_{0.75} \mathrm{I}_{3}$ Perovskites for Hysteresis-Free Solar Cells with Efficiency Reaching 15.2\%. Adv. Funct. Mater. 2017, 27, 1605469.

(172) Liu, J.; Ozaki, M.; Yakumaru, S.; Handa, T.; Nishikubo, R.; Kanemitsu, Y.; Saeki, A.; Murata, Y.; Murdey, R.; Wakamiya, A. Lead-Free Solar Cells Based on Tin Halide Perovskite 
Films with High Coverage and Improved Aggregation. Angew. Chem. Int. Ed. 2018, 57, 1322113225.

(173) Liu, X.; Yan, K.; Tan, D.; Liang, X.; Zhang, H.; Huang, W. Solvent Engineering Improves Efficiency of Lead-Free Tin-Based Hybrid Perovskite Solar Cells Beyond 9\%. ACS Energy Lett. 2018, 3, 2701-2707.

(174) Li, Y.; Sun, W.; Yan, W.; Ye, S.; Rao, H.; Peng, H.; Zhao, Z.; Bian, Z.; Liu, Z.; Zhou, H.; et al. 50\% Sn-Based Planar Perovskite Solar Cell with Power Conversion Efficiency up to 13.6\%. Adv. Energy Mater. 2016, 6, 1601353.

(175) Hong, W.-L.; Huang, Y.-C.; Chang, C.-Y.; Zhang, Z.-C.; Tsai, H.-R.; Chang, N.-Y.; Chao, Y.-C. Efficient Low-Temperature Solution-Processed Lead-Free Perovskite Infrared LightEmitting Diodes. Adv. Mater. 2016, 28, 8029-8036.

(176) Saidaminov, M. I.; Spanopoulos, I.; Abed, J.; Ke, W.; Wicks, J.; Kanatzidis, M. G.; Sargent E. H. Conventional Solvent Oxidizes Sn(II) in Perovskite Inks. ACS Energy Lett. 2020, 5, 1153-1155.

(177) Ahn, N.; Son, D.-Y.; Jang, I.-H.; Kang, S. M.; Choi, M.; Park, N.-G. Highly Reproducible Perovskite Solar Cells with Average Efficiency of 18.3\% and Best Efficiency of 19.7\% Fabricated via Lewis Base Adduct of Lead(II) Iodide. J. Am. Chem. Soc. 2015, 137, 8696-8699.

(178) Xi, J.; Wu, Z.; Jiao, B.; Dong, H.; Ran, C.; Piao, C.; Lei, T.; Song, T.-B.; Ke, W.; Yokoyama, T.; et al. Multichannel Interdiffusion Driven $\mathrm{FASnI}_{3}$ Film Formation Using Aqueous Hybrid Salt/Polymer Solutions toward Flexible Lead-Free Perovskite Solar Cells. Adv. Mater. 2017, 29 , 1606964.

(179) Moghe, D.; Wang, L.; Traverse, C. J.; Redoute, A.; Sponseller, M.; Brown, P. R.; VladimirBulović; Lunt, R. R. All Vapor-Deposited Lead-Free Doped CsSnBr 3 Planar Solar Cells. Nano Energy 2016, 28, 469-474.

(180) Tavakoli, M. M.; Zakeeruddin, S. M.; Grätzel, M.; Fan, Z. Large-Grain Tin-Rich Perovskite Films for Efficient Solar Cells via Metal Alloying Technique. Adv. Mater. 2018, 30, 1705998. (181) Ball, J. M.; Buizza, L.; Sansom, H. C.; Farrar, M. D.; Klug, M. T.; Borchert, J.; Patel, J.; Herz, L. M.; Johnston, M. B.; Snaith, H. J., Dual-Source Co-evaporation of Low-Bandgap $\mathrm{FA}_{1-\mathrm{x}} \mathrm{Cs}_{\mathrm{x}} \mathrm{Sn}_{1-\mathrm{y}} \mathrm{Pb}_{\mathrm{y}} \mathrm{I}_{3}$ Perovskites for Photovoltaics. ACS Energy Lett. 2019, 4, 2748-2756.

(182) He, L.; Gu, H., Liu, X.; Li, P.; Dang, Y.; Liang, C.; Ono, L. K.; Qi, Y.; Tao, X. Efficient Anti-solvent-free Spin-Coated and Printed Sn-Perovskite Solar Cells with Crystal-Based Precursor 
Solutions. Matter 2020, 2, 167-180.

(183) Kapil, G.; Bessho, T.; Ng, C. H.; Hamada, K.; Pandey, M.; Kamarudin, M. A.; Hirotani, D.; Kinoshita, T.; Minemoto, T.; Shen, Q.; et al. Strain Relaxation and Light Management in Tin-Lead Perovskite Solar Cells to Achieve High Efficiencies. ACS Energy Lett. 2019, 4, 1991-1998.

(184) Saliba, M.; Matsui, T.; Seo, J.-Y.; Domanski, K.; Correa-Baena, J.-P.; Nazeeruddin, M. K.; Zakeeruddin, S. M.; Tress, W.; Abate, A.; Hagfeldt, A.; et al. Cesium-Containing Triple Cation Perovskite Solar Cells: Improved Stability, Reproducibility and High Efficiency. Energy Environ. Sci. 2016, 9, 1989-1997.

(185) McMeekin, D. P.; Sadoughi, G.; Rehman, W.; Eperon, G. E.; Saliba, M.; Hörantner, M. T.; Haghighirad, A.; Sakai, N.; Korte, L.; Rech, B.; et al. A Mixed-Cation Lead Mixed-Halide Perovskite Absorber for Tandem Solar Cells. Science 2016, 351, 151-155.

(186) Gao, W.; Ran, C.; Li, J.; Dong, H.; Jiao, B.; Zhang, L.; Lan, X.; Hou, X.; Wu, Z. Robust Stability of Efficient Lead-Free Formamidinium Tin Iodide Perovskite Solar Cells Realized by Structural Regulation. J. Phys. Chem. Lett. 2018, 9, 6999-7006.

(187) Liao, W.; Zhao, D.; Yu, Y.; Shrestha, N.; Ghimire, K.; Grice, C. R.; Wang, C.; Xiao, Y.; Cimaroli, A. J.; Ellingson, R. J.; et al. Fabrication of Efficient Low-Bandgap Perovskite Solar Cells by Combining Formamidinium Tin Iodide with Methylammonium Lead Iodide. J. Am. Chem. Soc. 2016, 138, 12360-12363.

(188) Zong, Y.; Wang, N.; Zhang, L.; Ju, M.-G.; Zeng, X. C.; Sun, X. W.; Zhou, Y.; Padture, N. P. Homogenous Alloys of Formamidinium Lead Triiodide and Cesium Tin Triiodide for Efficient Ideal-Bandgap Perovskite Solar Cells. Angew. Chem. Int. Ed. 2017, 56, 12658-12662.

(189) Prasanna, R.; Parker, A.-G.; Leijtens, T.; Conings, B.; Babayigit, A.; Boyen, H.-G.; Toney, M. F.; McGehee, M. D. Band Gap Tuning Via Lattice Contraction and Octahedral Tilting in Perovskite Materials for Photovoltaics. J. Am. Chem. Soc. 2017, 139, 11117-11124.

(190) Zhao, Z.; Gu, F.; Li, Y.; Sun, W.; Ye, S.; Rao, H.; Liu, Z.; Bian, Z.; Huang, C. MixedOrganic-Cation Tin Iodide for Lead-Free Perovskite Solar Cells with an Efficiency of 8.12\%. Adv. Sci. 2017, 4, 1700204.

(191) Ogomi, Y.; Morita, A.; Tsukamoto, S.; Saitho, T.; Fujikawa, N.; Shen, Q.; Toyoda, T.; Yoshino, K.; Pandey, S. S.; Ma, T.; et al. $\mathrm{CH}_{3} \mathrm{NH}_{3} \mathrm{Sn}_{\mathrm{x}} \mathrm{Pb}_{1-\mathrm{x}} \mathrm{I}_{3}$ Perovskite Solar Cells Covering up to 1060 Nm. J. Phys. Chem. Lett. 2014, 5, 1004-1011.

(192) Li, M.; Wang, Z.-K.; Zhuo, M.-P.; Hu, Y.; Hu, K.-H.; Ye, Q.-Q.; Jain, S. M.; Yang, Y.-G.; 
Gao, X.-Y.; Liao, L.-S., Pb-Sn-Cu Ternary Organometallic Halide Perovskite Solar Cells. Adv. Mater. 2018, 30, 1800258.

(193) Yang, Z.; Rajagopal, A.; Jen, A. K.-Y. Ideal Bandgap Organic-Inorganic Hybrid Perovskite Solar Cells. Adv. Mater. 2017, 29, 1704418.

(194) Liang, J.; Zhao, P.; Wang, C.; Wang, Y.; Hu, Y.; Zhu, G.; Ma, L.; Liu, J.; Jin, Z. $\mathrm{CsPb}_{0.9} \mathrm{Sn}_{0.1} \mathrm{IBr}_{2}$ Based All-Inorganic Perovskite Solar Cells with Exceptional Efficiency and Stability. J. Am. Chem. Soc. 2017, 139, 14009-14012.

(195) Liu, J.; Wang, G.; Song, Z.; He, X.; Luo, K.; Ye, Q.; Liao, C.; Mei, J. FAPb ${ }_{1-x} \mathrm{Sn}_{\mathrm{x}} \mathrm{I}_{3}$ Mixed Metal Halide Perovskites with Improved Light Harvesting and Stability for Efficient Planar Heterojunction Solar Cells. J. Mater. Chem. A 2017, 5, 9097-9106.

(196) Yang, Z.; Rajagopal, A.; Jo, S. B.; Chueh, C.-C.; Williams, S.; Huang, C.-C.; Katahara, J. K.; Hillhouse, H. W.; Jen, A. K.-Y. Stabilized Wide Bandgap Perovskite Solar Cells by Tin Substitution. Nano Lett. 2016, 16, 7739-7747.

(197) Zuo, F.; Williams, S. T.; Liang, P.-W.; Chueh, C.-C.; Liao, C.-Y.; Jen, A. K.-Y. BinaryMetal Perovskites toward High-Performance Planar Heterojunction Hybrid Solar Cells. Adv. Mater. 2014, 26, 6454-6460.

(198) Zhao, D.; Yu, Y.; Wang, C.; Liao, W.; Shrestha, N.; Grice, C. R.; Cimaroli, A. J.; Guan, L.; Ellingson, R. J.; Zhu, K.; et al. Low-Bandgap Mixed Tin-Lead Iodide Perovskite Absorbers with Long Carrier Lifetimes for All-Perovskite Tandem Solar Cells. Nat. Energy 2017, 2, 17018.

(199) Ito, N.; Kamarudin, M. A.; Hirotani, D.; Zhang, Y.; Shen, Q.; Ogomi, Y.; Iikubo, S.; Minemoto, T.; Yoshino, K.; Hayase, S. Mixed Sn-Ge Perovskite for Enhanced Perovskite Solar Cell Performance in Air. J. Phys. Chem. Lett. 2018, 9, 1682-1688.

(200) Tsai, C.-M.; Mohanta, N.; Wang, C.-Y.; Lin, Y.-P.; Yang, Y.-W.; Wang, C.-L.; Hung, C.H.; Diau, E. W.-G. Formation of Stable Tin Perovskites Co-Crystallized with Three Halides for Carbon-Based Mesoscopic Lead-Free Perovskite Solar Cells. Angew. Chem. Int. Ed. 2017, 56, 13819-13823.

(201) Sabba, D.; Mulmudi, H. K.; Prabhakar, R. R.; Krishnamoorthy, T.; Baikie, T.; Boix, P. P.; Mhaisalkar, S.; Mathews, N. Impact of Anionic $\mathrm{Br}^{-}$Substitution on Open Circuit Voltage in Lead Free Perovskite $\left(\mathrm{CsSnI}_{3-\mathrm{x}} \mathrm{Br}_{\mathrm{x}}\right)$ Solar Cells. J. Phys. Chem. C 2015, 119, 1763-1767.

(202) Ke, W.; Stoumpos, C. C.; Logsdon, J. L.; Wasielewski, M. R.; Yan, Y.; Fang, G.; Kanatzidis, M. G. Tio $_{2}-\mathrm{ZnS}$ Cascade Electron Transport Layer for Efficient Formamidinium Tin Iodide 
Perovskite Solar Cells. J. Am. Chem. Soc. 2016, 138, 14998-15003.

(203) Wang, N.; Zhou, Y.; Ju, M.-G.; Garces, H. F.; Ding, T.; Pang, S.; Zeng, X. C.; Padture, N. P.; Sun, X. W. Heterojunction-Depleted Lead-Free Perovskite Solar Cells with Coarse-Grained B$\Gamma-\mathrm{CsSnI}_{3}$ Thin Films. Adv. Energy Mater. 2016, 6, 1601130.

(204) Chi, D.; Huang, S.; Zhang, M.; Mu, S.; Zhao, Y.; Chen, Y.; You, J. Composition and Interface Engineering for Efficient and Thermally Stable Pb-Sn Mixed Low-Bandgap Perovskite Solar Cells. Adv. Funct. Mater. 2018, 28, 1804603.

(205) Shao, S.; Cui, Y.; Duim, H.; Qiu, X.; Dong, J.; Brink, G. H. t.; Portale, G.; Chiechi, R. C.; Zhang, S.; Hou, J.; et al. Enhancing the Performance of the Half Tin and Half Lead Perovskite Solar Cells by Suppression of the Bulk and Interfacial Charge Recombination. Adv. Mater. 2018, 30, 1803703.

(206) Prasanna, R.; Leijtens, T.; Dunfield, S. P.; Raiford, J. A.; Wolf, E. J.; Swifter, S. A.; Werner, J.; Eperon, G. E.; Paula, C. d.; Palmstrom, A. F.; et al. Design of Low Bandgap Tin-Lead Halide Perovskite Solar Cells to Achieve Thermal, Atmospheric and Operational Stability. Nat. Energy 2019, 4, 939-947.

(207) Vegiraju, S.; Ke, W.; Priyanka, P.; Ni, J.-S.; Wu, Y.-C.; Spanopoulos, I.; Yau, S. L.; Marks, T. J.; Chen, M.-C.; Kanatzidis, M. G. Benzodithiophene Hole-Transporting Materials for Efficient Tin-Based Perovskite Solar Cells. Adv. Funct. Mater. 2019, 29, 1905393.

(208) Liu, X.; Wang, Y.; Xie, F.; Yang, X.; Han, L. Improving the Performance of Inverted Formamidinium Tin Iodide Perovskite Solar Cells by Reducing the Energy-Level Mismatch. ACS Energy Lett. 2018, 3, 1116-1121.

(209) Xu, G.; Bi, P.; Wang, S.; Xue, R.; Zhang, J.; Chen, H.; Chen, W.; Hao, X.; Li, Y.; Li, Y. Integrating Ultrathin Bulk-Heterojunction Organic Semiconductor Intermediary for HighPerformance Low-Bandgap Perovskite Solar Cells with Low Energy Loss. Adv. Funct. Mater. 2018, 28, 1804427.

(210) Chen, K.; Wu, P.; Yang, W.; Su, R.; Luo, D.; Yang, X.; Tu, Y.; Zhu, R.; Gong, Q. LowDimensional Perovskite Interlayer for Highly Efficient Lead-Free Formamidinium Tin Iodide Perovskite Solar Cells. Nano Energy 2018, 49, 411-418.

(211) Ran, C.; Xi, J.; Gao, W.; Yuan, F.; Lei, T.; Jiao, B.; Hou, X.; Wu, Z. Bilateral Interface Engineering toward Efficient 2D-3D Bulk Heterojunction Tin Halide Lead-Free Perovskite Solar Cells. ACS Energy Lett. 2018, 3, 713-721. 
(212) Lin, G.; Lin, Y.; Huang, H.; Cui, R.; Guo, X.; Liu, B.; Dong, J.; Guo, X.; Sun, B. Novel Exciton Dissociation Behavior in Tin-Lead Organohalide Perovskites. Nano Energy 2016, 27, 638-646.

(213) Marshall, K. P.; Walton, R. I.; Hatton, R. A. Tin Perovskite/Fullerene Planar Layer Photovoltaics: Improving the Efficiency and Stability of Lead-Free Devices. J. Mater. Chem. A 2015, 3, 11631-11640.

(214) Kapil, G.; Ripolles, T. S.; Hamada, K.; Ogomi, Y.; Bessho, T.; Kinoshita, T.; Chantana, J.; Yoshino, K.; Shen, Q.; Toyoda, T.; et al. Highly Efficient 17.6\% Tin-Lead Mixed Perovskite Solar Cells Realized through Spike Structure. Nano Lett. 2018, 18, 3600-3607.

(215) Yang, Z.; Zhong, M.; Liang, Y.; Yang, L.; Liu, X.; Li, Q.; Zhang, J.; Xu, D. SnO ${ }_{2}-\mathrm{C}_{60}$ Pyrrolidine Tris-Acid (CPTA) as the Electron Transport Layer for Highly Efficient and Stable Planar Sn-Based Perovskite Solar Cells. Adv. Funct. Mater. 2019, 29, 1903621.

(216) Liu, M.; Chen, Z.; Yang, Y.; Yip, H.-L.; Cao, Y., Reduced Open-Circuit Voltage Loss for Highly Efficient Low-Bandgap Perovskite Solar Cells Via Suppression of Silver Diffusion. J. Mater. Chem. A 2019, 7, 17324-17333.

(217) Boehm, A. M.; Liu, T.; Park, S. M.; Abtahi, A.; Graham, K. R. Influence of Surface Ligands on Energetics at $\mathrm{FASnI}_{3} / \mathrm{C}_{60}$ Interfaces and Their Impact on Photovoltaic Performance. ACS Appl. Mater. Interfaces 2020, 12, 5209-5218.

(218) Ke, W.; Spanopoulos, I.; Tu, Q.; Hadar, I.; Li, X.; Shekhawat, G. S.; Dravid, V. P.; Kanatzidis, M. G. Ethylenediammonium-Based "Hollow” Pb/Sn Perovskites with Ideal Band Gap Yield Solar Cells with Higher Efficiency and Stability. J. Am. Chem. Soc. 2019, 141, 8627-8637. (219) Spanopoulos, I.; Ke, W.; Stoumpos, C. C.; Schueller, E. C.; Kontsevoi, O. Y.; Seshadri, R.; Kanatzidis, M. G. Unraveling the Chemical Nature of the 3D "Hollow" Hybrid Halide Perovskites. J. Am. Chem. Soc. 2018, 140, 5728-5742.

(220) Zimmermann, I.; Aghazada, S.; Nazeeruddin, M. K. Lead and HTM Free Stable TwoDimensional Tin Perovskites with Suitable Band Gap for Solar Cell Applications. Angew. Chem. Int. Ed. 2019, 58, 1072-1076.

(221) Liu, F.; Ding, C.; Zhang, Y.; Ripolles, T. S.; Kamisaka, T.; Toyoda, T.; Hayase, S.; Minemoto, T.; Yoshino, K.; Dai, S.; et al. Colloidal Synthesis of Air-Stable Alloyed $\mathrm{CsSn}_{1-\mathrm{x}} \mathrm{Pb}_{\mathrm{x}} \mathrm{I}_{3}$ Perovskite Nanocrystals for Use in Solar Cells. J. Am. Chem. Soc. 2017, 139, 16708-16719. (222) Liu, F.; Zhang, Y.; Ding, C.; Kawabata, K.; Yoshihara, Y.; Toyoda, T.; Hayase, S.; 
Minemoto, T.; Wang, R.; Shen, Q. Trioctylphosphine Oxide Acts as Alkahest for $\mathrm{SnX}_{2} / \mathrm{PbX}_{2}$ : A General Synthetic Route to Perovskite $\mathrm{ASn}_{\mathrm{X}} \mathrm{Pb}_{1-\mathrm{X}} \mathrm{X}_{3}(\mathrm{~A}=\mathrm{Cs}, \mathrm{FA}, \mathrm{MA} ; \mathrm{X}=\mathrm{Cl}, \mathrm{Br}$, I) Quantum Dots. Chem. Mater. 2020, 32, 1089-1100.

(223) Cao, D. H.; Stoumpos, C. C.; Yokoyama, T.; Logsdon, J. L.; Song, T.-B.; Farha, O. K.; Wasielewski, M. R.; Hupp, J. T.; Kanatzidis, M. G. Thin Films and Solar Cells Based on Semiconducting Two-Dimensional Ruddlesden-Popper $\left(\mathrm{CH}_{3}\left(\mathrm{CH}_{2}\right)_{3} \mathrm{NH}_{3}\right)_{2}\left(\mathrm{CH}_{3} \mathrm{NH}_{3}\right)_{\mathrm{n}-1} \mathrm{Sn}_{\mathrm{n}} \mathrm{I}_{3 \mathrm{n}+1}$ Perovskites. ACS Energy Lett. 2017, 2, 982-990.

(224) Ramirez, D.; Schutt, K.; Wang, Z.; Pearson, A. J.; Ruggeri, E.; Snaith, H. J.; Stranks, S. D.; Jaramillo, F. Layered Mixed Tin-Lead Hybrid Perovskite Solar Cells with High Stability. ACS Energy Lett. 2018, 3, 2246-2251.

(225) Liao, Y.; Liu, H.; Zhou, W.; Yang, D.; Shang, Y.; Shi, Z.; Li, B.; Jiang, X.; Zhang, L.; Quan, L. N.; et al. Highly Oriented Low-Dimensional Tin Halide Perovskites with Enhanced Stability and Photovoltaic Performance. J. Am. Chem. Soc. 2017, 139, 6693-6699.

(226) Chen, M.; Ju, M.-G.; Hu, M.; Dai, Z.; Hu, Y.; Rong, Y.; Han, H.; Zeng, X. C.; Zhou, Y.; Padture, N. P. Lead-Free Dion-Jacobson Tin Halide Perovskites for Photovoltaics. ACS Energy Lett. 2019, 4, 276-277.

(227) Zeng, L.; Chen, Z.; Qiua, S.; Hu, J.; Li, C.; Liu, X.; Liang, G.; Brabece, C. J.; Mai, Y.; Guo, F. 2D-3D Heterostructure Enables Scalable Coating of Efficient Low-Bandgap Sn-Pb Mixed Perovskite Solar Cells. Nano Energy 2019, 66, 104099.

(228) Ran, C.; Gao, W.; Li, J.; Xi, J.; Li, L.; Dai, J.; Yang, Y.; Gao, X.; Dong, H.; Jiao, B.; et al. Conjugated Organic Cations Enable Efficient Self-Healing FASnI 3 Solar Cells. Joule 2018, 3, 3072-3087.

(229) Xu, H.; Jiang, Y.; He, T.; Li, S.; Wang, H.; Chen, Y.; Yuan, M.; Chen, J. Orientation Regulation of Tin-Based Reduced-Dimensional Perovskites for Highly Efficient and Stable Photovoltaics. Adv. Funct. Mater. 2019, 29, 1807696.

(230) Shao, S.; Liu, J.; Portale, G.; Fang, H.-H.; Blake, G. R.; Brink, G. H. t.; Koster L. J. A.; Loi, M. A. Highly Reproducible Sn-Based Hybrid Perovskite Solar Cells with 9\% Efficiency. Adv. Energy Mater. 2018, 8, 1702019.

(231) Liu, C.; Tu, J.; Hu, X.; Huang, Z.; Meng, X.; Yang, J.; Duan, X.; Tan, L.; Li Z.; Chen, Y. Enhanced Hole Transportation for Inverted Tin-Based Perovskite Solar Cells with High Performance and Stability. Adv. Funct. Mater. 2019, 29, 1808059. 
(232) Mehta, A.; Im, J.; Kim, B. H.; Min, H.; Nie, R.; Seok, S. I. Stabilization of Lead-Tin-Alloyed Inorganic-Organic Halide Perovskite Quantum Dots. ACS Nano 2018, 12, 12129-12139.

(233) Bowman, A. R.; Klug, M. T.; Doherty, T. A. S.; Farrar, M. D.; Senanayak, S. P.; Wenger, B.; Divitini, G.; Booker, E. P.; Andaji-Garmaroudi, Z.; Macpherson, S.; et al. Microsecond Carrier Lifetimes, Controlled p-Doping, and Enhanced Air Stability in Low-Bandgap Metal Halide Perovskites. ACS Energy Lett. 2019, 4, 2301-2307.

(234) Xing, G.; Kumar, M. H.; Chong, W. K.; Liu, X.; Cai, Y.; Ding, H.; Asta, M.; Grätzel, M.; Mhaisalkar, S.; Mathews, N.; et al. Solution-Processed Tin-Based Perovskite for Near-Infrared Lasing. Adv. Mater. 2016, 28, 8191-8196.

(235) Zhang, X.; Cao, W.; Wang, W.; Xu, B.; Liu, S.; Dai, H.; Chen, S.; Wang, K.; Sun, X. W. Efficient Light-Emitting Diodes Based on Green Perovskite Nanocrystals with Mixed-Metal Cations. Nano Energy 2016, 30, 511-516.

(236) Lai, M. L.; Tay, T. Y. S.; Sadhanala, A.; Dutton, S. n. E.; Li, G.; Friend, R. H.; Tan, Z.-K. Tunable Near-Infrared Luminescence in Tin Halide Perovskite Devices. J. Phys. Chem. Lett. 2016, 7, 2653-2658.

(237) Wang, H.-C.; Wang, W.; Tang, A.-C.; Tsai, H.-Y.; Bao, Z.; Ihara, T.; Yarita, N.; Tahara, H.; Kanemitsu, Y.; Chen, S.; et al. High-Performance $\mathrm{CsPb}_{1-\mathrm{x}} \mathrm{Sn}_{\mathrm{x}} \mathrm{Br}_{3}$ Perovskite Quantum Dots for Light-Emitting Diodes. Angew. Chem. Int. Ed. 2017, 56, 13650-13654.

(238) Wu, Z.; Zhang, Q.; Li, B.; Shi, Z.; Xu, K.; Chen, Y.; Ning, Z.; Mi, Q. Stabilizing the $\mathrm{CsSnCl}_{3}$ Perovskite Lattice by B-Site Substitution for Enhanced Light Emission. Chem. Mater. 2019, 31, 4999-5004.

(239) Lanzetta, L.; Marin-Beloqui, J. M.; Sanchez-Molina, I.; Ding, D.; Haque, S. A. TwoDimensional Organic Tin Halide Perovskites with Tunable Visible Emission and Their Use in Light-Emitting Devices. ACS Energy Lett. 2017, 2, 1662-1668.

(240) Zhou, C.; Lin, H.; Tian, Y.; Yuan, Z.; Clark, R.; Chen, B.; Burgt, L. J. v. d.; Wang, J. C.; Zhou, Y.; Hanson, K.; et al. Luminescent Zero-Dimensional Organic Metal Halide Hybrids with near-Unity Quantum Efficiency. Chem. Sci. 2018, 9, 586-593.

(241) Morad, V.; Shynkarenko, Y.; Yakunin, S.; Brumberg, A.; Schaller, R. D.; Kovalenko, M. V. Disphenoidal Zero-Dimensional Lead, Tin, and Germanium Halides: Highly Emissive Singlet and Triplet Self-Trapped Excitons and X-Ray Scintillation. J. Am. Chem. Soc. 2019, 141, 9764-9768. (242) Lin, J.-T.; Liao, C.-C.; Hsu, C.-S.; Chen, D.-G.; Chen, H.-M.; Tsai, M.-K.; Chou, P.-T.; 
Chiu, C.-W. Harnessing Dielectric Confinement on Tin Perovskites to Achieve Emission Quantum Yield up to 21\%. J. Am. Chem. Soc. 2019, 141, 10324-10330.

(243) Li, J.; Stoumpos, C. C.; Trimarchi, G. G.; Chung, I.; Mao, L.; Chen, M.; Wasielewski, M. R.; Wang, L.; Kanatzidis, M. G. Air-Stable Direct Bandgap Perovskite Semiconductors: AllInorganic Tin-Based Heteroleptic Halides $\mathrm{A}_{\mathrm{x}} \mathrm{SnCl}_{\mathrm{y}} \mathrm{Iz}(\mathrm{A}=\mathrm{Cs}, \mathrm{Rb})$. Chem. Mater. 2018, 30, 48474856.

(244) Zhu, H. L.; Liang, Z.; Huo, Z.; Ng, W. K.; Mao, J.; Wong, K. S.; Yin, W.-J.; Choy, W. C. H. Low-Bandgap Methylammonium-Rubidium Cation Sn-Rich Perovskites for Efficient Ultraviolet-Visible-Near Infrared Photodetectors. Adv. Funct. Mater. 2018, 28, 1706068.

(245) Fang, C.; Wang, H.; Shen, Z.; Shen, H.; Wang, S.; Ma, J.; Wang, J.; Luo, H.; Li, D. HighPerformance Photodetectors Based on Lead-Free 2D Ruddlesden-Popper Perovskite/MoS 2 Heterostructures. ACS Appl. Mater. Interfaces 2019, 11, 8419-8427.

(246) Zhu, H.; Liu, A.; Luque, H. L.; Sun, H.; Ji, D.; Noh, Y.-Y. Perovskite and Conjugated Polymer Wrapped Semiconducting Carbon Nanotube Hybrid Films for High-Performance Transistors and Phototransistors. ACS Nano 2019, 13, 3971-3981.

(247) Zhu, H. L.; Lin, H.; Song, Z.; Wang, Z.; Ye, F.; Zhang, H.;Yin, W.-J.; Yan, Y.; Choy, W. C. H. Achieving High-Quality $\mathrm{Sn}-\mathrm{Pb}$ Perovskite Films on Complementary Metal-OxideSemiconductor-Compatible Metal/Silicon Substrates for Efficient Imaging Array. ACS Nano 2019, 13, 11800-11808.

(248) Waleed, A.; Tavakoli, M. M.; Gu, L.; Wang, Z.; Zhang, D.; Manikandan, A.; Zhang, Q.; Zhang, R.; Chueh, Y.-L.; Fan, Z. Lead-Free Perovskite Nanowire Array Photodetectors with Drastically Improved Stability in Nanoengineering Templates. Nano Lett. 2017, 17, 523-530.

(249) Park, I.-H.; Chu, L.; Leng, K.; Choy, Y. F.; Liu, W.; Abdelwahab, I.; Zhu, Z.; Ma, Z.; Chen, W.; Xu, Q.-H.; et al. Highly Stable Two-Dimensional Tin(II) Iodide Hybrid Organic-Inorganic Perovskite Based on Stilbene Derivative. Adv. Funct. Mater. 2019, 29, 1904810.

(250) Chen, L.-J.; Dai, J.-H.; Lin, J.-D.; Mo, T.-S.; Lin, H.-P.; Yeh, H.-C.; Chuang, Y.-C.; Jiang, S.-A.; Lee, C.-R. Wavelength-Tunable and Highly Stable Perovskite-Quantum-Dot-Doped Lasers with Liquid Crystal Lasing Cavities. ACS Appl. Mater. Interfaces 2018, 10, 33307-33315.

(251) Sun, Y.; Qian, L.; Xie, D.; Lin, Y.; Sun, M.; Li, W.; Ding, L.; Ren, T.; Palacios, T. Photoelectric Synaptic Plasticity Realized by 2D Perovskite. Adv. Funct. Mater. 2019, 29, 1902538. 
(252) Qian, L.; Sun, Y.; Wu, M.; Li, C.; Xie, D.; Ding, L.; Shi, G. A Lead-Free Two-Dimensional Perovskite for a High-Performance Flexible Photoconductor and a Light-Stimulated Synaptic Device. Nanoscale 2018, 10, 6837-6843.

(253) Pandey, R.; SB, G.; Grover, S.; Singh, S. K.; Kadam, A.; Ogale, S.; Waghmare, U. V.; Rao, V. R.; Kabra, D. Microscopic Origin of Piezoelectricity in Lead-Free Halide Perovskite: Application in Nanogenerator Design. ACS Energy Lett. 2019, 4, 1004-1011.

(254) Ippilia, S.; Jellaa, V.; Eoma, J.-H.; Kimb, J.; Hongb, S.; Choic, J.-S.; Trana, V.-D.; Hieud, N. V.; Kime, Y.-J.; Kime, H.-J.; et al. An Eco-Friendly Flexible Piezoelectric Energy Harvester That Delivers High Output Performance Is Based on Lead-Free MASnI ${ }_{3}$ Films and MASnI 3 -PVDF Composite Films. Nano Energy 2019, 57, 911-923.

(255) Ju, D.; Zheng, X.; Liu, J.; Chen, Y.; Zhang, J.; Cao, B.; Xiao, H.; Mohammed, O. F.; Bakr, O. M.; Tao, X. Reversible Band Gap Narrowing of Sn-Based Hybrid Perovskite Single Crystal with Excellent Phase Stability. Angew. Chem. Int. Ed. 2018, 57, 14868-14872.

(256) Matsushima, T.; Mathevet, F.; Heinrich, B.; Terakawa, S.; Fujihara, T.; Qin, C.; Sandanayaka, A. S. D.; Ribierre, J.-C.; Adachi, C. N-Channel Field-Effect Transistors with an Organic-Inorganic Layered Perovskite Semiconductor. Appl. Phys. Lett. 2016, 109, 253301. (257) Gao, Y.; Wei, Z.; Yoo, P.; Shi, E.; Zeller, M.; Zhu, C.; Liao, P.; Dou, L. Highly Stable LeadFree Perovskite Field-Effect Transistors Incorporating Linear $\pi$-Conjugated Organic Ligands. $J$. Am. Chem. Soc. 2019, 141, 15577-15585. 


\section{Quotes}

1. When delving into the fundamentals of electronic structure, photophysics, and degradation pathways as elucidated above, the intrinsic issues in Sn-PVSKs emerge as 1) easy oxidation of $\mathrm{Sn}^{2+}$, which impairs long-term structural stability; 2) oxidation induced p-doping, which causes severe monomolecular recombination and $E_{\mathrm{g}}$ widening; 3) mismatched band alignment between the active layer and the transporting layers, which hinders the collection of free carriers; 4) rapid and uncontrollable crystallization, which present a critical technical challenge towards high-quality preparation of Sn-PVSK thin films. (Page 26)

2. To sum, the mixed cation strategy is a viable avenue to encompass all the merits of single Asite cation at one strike. Various functional groups such as reducing double bonds or coordinating endings may be worth to incorporate into their molecule structures to tailor the optoelectronic properties and stability of $\mathrm{ASnX}_{3}$ provided that the tolerance factor is satisfied. (Page 44)

3. In order to pinpoint appropriate additives, the Hard-Soft-Acid-Base theory may be useful. Since $\mathrm{Sn}^{2+}$ belongs to the borderline acid, it can coordinate with either hard or soft bases, which include a wide range of options, to name a few, $\mathrm{SO}_{3}^{-}$(hard base) and $\mathrm{SCN}^{-}$(soft base). Importantly, the shielding and un-shielding effects may provide additional inspirations. (Page 68)

4. In this scenario, the basic properties of solvents such as miscibility with good solvent, viscosity and volatility all deserve careful considerations. The mixed anti-solvent strategy seems a viable approach. Moreover, if the additives (either crystallization regulator or antioxidants) can be incorporated in to the antisolvent, undesirable film forming and oxidation during preparation may also be effectively modulated and suppressed. (Page 69) 